\title{
ULTRADIFFERENTIABLE EXTENSION THEOREMS: A SURVEY
}

\author{
ARMIN RAINER
}

\begin{abstract}
We survey ultradifferentiable extension theorems, i.e., quantitative versions of Whitney's classical extension theorem, with special emphasis on the existence of continuous linear extension operators. The focus is on Denjoy-Carleman classes for which we develop the theory from scratch and discuss important related concepts such as (non-)quasianalyticity. It allows us to give an efficient and, to a fair extent, elementary introduction to BraunMeise-Taylor classes based on their representation as intersections and unions of Denjoy-Carleman classes.
\end{abstract}

\section{Contents}

1. Introduction

Part 1. Denjoy-Carleman classes 5

2. Ultradifferentiable functions 5

3. The Borel map and the Denjoy-Carleman theorem 10

4. Surjectivity of the Borel map $\quad 15$

5. The Borel map in the quasianalytic setting 21

6. Borel's lemma with controlled loss of regularity 24

7. Optimal cutoff functions 26

8. Extension of Whitney ultrajets 33

9. Continuous linear extension operators 38

10. Extension with controlled loss of regularity 45

Part 2. More general ultradifferentiable classes 50

11. Further ultradifferentiable functions $\quad 50$

12. Extension in Braun-Meise-Taylor classes 60

13. Extensions not preserving the ultradifferentiable class 65

References $\quad 68$

Date: January 3, 2022.

2020 Mathematics Subject Classification. 26E10, 30D60, 46E10, 46E25, 46A63, 58C25 .

Key words and phrases. Whitney's extension theorem, Borel map, (non-)quasianalyticity, ultradifferentiable classes, extension operators.

The author was supported by the Austrian Science Fund (FWF), Grant P 32905-N and START Programme Y963. 


\section{INTRODUCTION}

The development of differential analysis in the last century was strongly influenced by Whitney's work on differentiable functions. Especially fruitful was Whitney's extension theorem which describes the restrictions of smooth functions on $\mathbb{R}^{n}$ to closed subsets. For a closed non-empty set $A \subseteq \mathbb{R}^{n}$ we consider the restriction map

$$
j_{A}^{\infty}: C^{\infty}\left(\mathbb{R}^{n}\right) \rightarrow C^{0}(A)^{\mathbb{N}^{n}}, \quad f \mapsto\left(\left.f^{(\alpha)}\right|_{A}\right)_{\alpha \in \mathbb{N}^{n}}
$$

Taylor's theorem implies that the image $j_{A}^{\infty} C^{\infty}\left(\mathbb{R}^{n}\right)$ is contained in the set $\mathcal{E}(A)$ of Whitney jets: $F=\left(F^{\alpha}\right)_{\alpha} \in C^{0}(A)^{\mathbb{N}^{n}}$ belongs to $\mathcal{E}(A)$ if for all compact subsets $K \subseteq A$, all $p \in \mathbb{N}$, and all $|\alpha| \leq p$

$$
\left(R_{x}^{p} F\right)^{\alpha}(y)=o\left(|x-y|^{p-|\alpha|}\right) \quad \text { as }|x-y| \rightarrow 0, x, y \in K
$$

where

$$
\left(R_{x}^{p} F\right)^{\alpha}(y):=F^{\alpha}(y)-\sum_{|\beta| \leq p-|\alpha|} \frac{(y-x)^{\beta}}{\beta !} F^{\alpha+\beta}(x) .
$$

Whitney's extension theorem [92] states that $j_{A}^{\infty} C^{\infty}\left(\mathbb{R}^{n}\right)=\mathcal{E}(A)$, i.e., every Whitney jet $F \in \mathcal{E}(A)$ admits an extension $f \in C^{\infty}\left(\mathbb{R}^{n}\right)$ such that $j_{A}^{\infty} f=F$.

In this paper we shall be interested in quantitative versions of Whitney's extension theorem.

Question 1.1. Given that a Whitney jet $F \in \mathcal{E}(A)$ satisfies certain uniform growth properties, can these properties be preserved by the extension? If they cannot be preserved, can the loss of regularity be controlled? Can the extension be performed by a continuous linear map?

The uniform growth properties we have in mind are bounds on the multisequence of partial derivatives imposed in terms of a suitable weight sequence which measures the deviation from the Cauchy estimates and hence from analyticity. They give rise to so-called ultradifferentiable classes which form scales of regularity classes between the real analytic and the smooth class. More specifically, the use of a weight sequence $M$ leads to Denjoy-Carleman classes which originated around 1900 in work of Borel, Gevrey, Holmgren, Hadamard, etc. A different approach based on decay properties of the Fourier transform is due to Beurling and Björck in the 1960s. An equivalent description of this second approach was later given by Braun, Meise, and Taylor. The resulting classes are called Braun-Meise-Taylor classes; their definition involves a weight function $\omega$.

In this survey we will first treat the extension problem extensively for DenjoyCarleman classes. In a second part we will discuss it for Braun-Meise-Taylor classes. Our treatment of the latter is based on their description as intersections and unions of Denjoy-Carleman classes in terms of an associated family of weight sequences (called weight matrix).

Our study of Question 1.1 will first focus on the simplest case when the closed set $A$ is just the singleton $\{0\}$. Then $j_{\{0\}}^{\infty}$ is called Borel map, $\mathcal{E}(\{0\})$ is isomorphic to the ring of formal power series $\mathbb{K}\left[\left[X_{1}, \ldots, X_{n}\right]\right]$ (where $\mathbb{K}$ is $\mathbb{R}$ or $\mathbb{C}$ ), and Whitney's extension theorem reduces to Borel's lemma. Émile Borel's discovery at the end of the 19th century that there exist classes of smooth functions which have an analytic continuation property (called quasianalyticity), but contain functions that are nowhere analytic, led to a lot of activity. A class of smooth functions is quasianalytic if the restriction of the Borel map to this class is injective. 
The famous Denjoy-Carleman theorem characterizes quasianalyticity of DenjoyCarleman classes in terms of the weight sequence $M$. We give a full proof based on two elementary lemmas (Lemma 3.2 and Lemma 3.3) which will turn out to be foundational for the whole theory. For example, we will deduce the characterization of quasianalyticity for Braun-Meise-Taylor classes from the Denjoy-Carleman theorem.

The bounds on the derivatives defining an ultradifferentiable class naturally induce corresponding bounds for the infinite jet of such functions at 0 . Thus the natural codomain of the Borel map on an ultradifferentiable class is a sequence space defined by the corresponding bounds, and one may ask about surjectivity of this map. We will see that surjectivity on Denjoy-Carleman classes is equivalent to a condition on the weight sequence $M$ that is called strong non-quasianalyticity. It will turn out that this condition is indispensable for the existence of suitable (even optimal) cutoff functions crucial for the solution of the extension problem. By different methods (studying the distribution of zeros of quasianalytic functions and their derivatives) we shall also see that the Borel map is never surjective in the quasianalytic setting, except for the real analytic class.

All ultradifferentiable classes considered in this paper come in two types, Beurling type and Roumieu type. The classes of Beurling type carry a natural locally convex topology that make them to Fréchet spaces, the topology of the classes of Roumieu type is more complicated. We shall see that the Borel map on DenjoyCarleman classes of Beurling type is even split surjective if $M$ is strongly nonquasianalytic. In the Roumieu case, generally, the Borel map does not admit a continuous linear right-inverse.

Having solved the extension problem for the Borel map, the solution of the general problem for arbitrary non-empty closed subsets $A$ of $\mathbb{R}^{n}$ depends on the existence of optimal cutoff functions. They are optimal in the sense that they realize necessary sharp bounds. In conjunction with a family of Whitney cubes for $A$, they yield optimal partitions of unity with the help of which the local extensions (provided by the solution for the Borel map) can be glued to a global extension of Whitney ultrajets on $A$. The construction of these cutoff functions involves some delicate properties of auxiliary functions associated with the weight sequence $M$.

The existence of extensions in the Beurling case follows by a reduction argument from the Roumieu case. But we will also find optimal cutoff functions of Beurling type and utilize them for a direct proof of the split surjectivity of the restriction map $j_{A}^{\infty}$ on Denjoy-Carleman classes of Beurling type. In this way we give an elementary constructive proof for the existence of extension operators in this setting, without relying on the abstract splitting theorem for Fréchet spaces, which we shall however discuss briefly.

If strong non-quasianalyticity is lacking, and hence extension preserving the class is impossible, one is led to the problem of controlling the loss of regularity. This problem is solved by describing the pairs of weight sequences $(M, N)$ (later called admissible pairs) such that Whitney jets on a closed set $A$ satisfying $M$ bounds admit extensions with $N$-bounds, both in the Beurling and Roumieu case. Technically, this requires an interesting tool inspired by Dyn'kin's theory of almost analytic functions: instead of the local extensions (for the singleton) one uses the Taylor polynomials of the jet to higher and higher degree as the closed set $A$ is approached. 
The second part of the survey is dedicated to a concise introduction to BraunMeise-Taylor classes and a discussion of the extension problem in that framework. Our approach is based on the description of these classes as suitable intersections and unions of Denjoy-Carleman classes which allows (to a fair extent) for a swift and elementary treatment, building on the extensive study of the latter in part one. The discussion of the extension problem for Braun-Meise-Taylor classes will be more expository (compared to part one). We will present the state of the art of this area mostly without proofs but indicating the important ideas and methods involved. Interestingly, there appear phenomena that are not present in the framework of Denjoy-Carleman classes. For instance, if extensions preserving the class are possible, even in the Beurling case they cannot always be realized by extension operators. The existence of extension operators (always in the Beurling case) depends on the geometry of the set $A$ and on the weight function $\omega$. The singleton $\{0\}$, for example, admits an extension operator if and only if $\omega$ has a certain additional property (namely, it is a so-called (DN)-weight). And, if $\omega$ has this property, then every closed set $A$ has an extension operator. Given that $\omega$ lacks that property, then the existence of an extension operator on a compact set $K$ is characterized by the linear topological invariant (DN) of the space of Whitney $\omega$-ultrajets of Beurling type on $K$. A compact set may or may not satisfy this condition: sets with real analytic boundary do and sets with sharp (i.e., infinitely flat) cusps do not.

In order to make the exposition not too technical we do not strive for the utmost generality of the results. But we provide appropriate references for the interested reader.

This survey article arose from notes for a mini-course I gave at the School of Real Geometry in Fortaleza, Brazil, May 24-28, $2021 .{ }^{1}$

Notation. We use standard multiindex notation.

The supremum norm is denoted by $\|u\|_{K}:=\sup _{x \in K}|u(x)|$.

A sequence $\left(a_{k}\right)$ is called increasing if $a_{k} \leq a_{k+1}$ and strictly increasing if $a_{k}<$ $a_{k+1}$ for all $k$; analogously with decreasing. That an increasing sequence $\left(a_{k}\right)$ tends to infinity is abbreviated by $a_{k} \nearrow \infty$.

For two non-negative sequences $a=\left(a_{k}\right)$ and $b=\left(b_{k}\right)$ we write $a \lesssim b$ if there is a constant $C>0$ such that $a_{k} \leq C b_{k}$ for all $k$; similarly for functions. We will also use $a \preccurlyeq b$ for $a_{k}^{1 / k} \lesssim b_{k}^{1 / k}$ and $a \triangleleft b$ for $a_{k}^{1 / k} / b_{k}^{1 / k} \rightarrow 0$.

The indicator function of a subset $A \subseteq \mathbb{R}^{n}$ is denoted by $\mathbf{1}_{A}$. If $A$ is nonempty, then $\operatorname{diam} A:=\sup \{|a-b|: a, b \in A\}$ is the diameter of $A$. If $B \subseteq \mathbb{R}^{n}$ is another non-empty set, then $\operatorname{dist}(A, B):=\inf \{|a-b|: a \in A, b \in B\}$ is the Euclidean distance between $A$ and $B$. In particular, $d_{A}(z)=d(z, A)=\operatorname{dist}(z, A):=$ $\operatorname{dist}(\{z\}, A)$ for $z \in \mathbb{R}^{n}$.

For open subsets $A, B \subseteq \mathbb{R}^{n}$ we use the symbol $A \Subset B$ to indicate that $A$ is relatively compact in $B$. And we write $K \subseteq_{c p} A$ if $K$ is a compact subset of $A$.

\footnotetext{
$1_{\text {https }}$ //sites.google.com/view/scregefor $2020 /$
} 


\section{Part 1. Denjoy-Carleman classes}

\section{ULtRADIFFERENTIABLE FUNCTIONS}

In this section we discuss natural growth conditions for the infinite sequence of derivatives of smooth functions.

2.1. Denjoy-Carleman classes. Let $M=\left(M_{k}\right)_{k \in \mathbb{N}}$ be a sequence of positive real numbers. Let $U$ be an open subset of $\mathbb{R}^{n}$. For $f \in C^{\infty}(U), \rho>0$, and compact $K \subseteq U$ we consider the seminorm

$$
\|f\|_{K, \rho}^{M}:=\sup _{x \in K} \sup _{\alpha \in \mathbb{N}^{n}} \frac{\left|f^{(\alpha)}(x)\right|}{\rho^{|\alpha|} M_{|\alpha|}}=\sup _{\alpha \in \mathbb{N}^{n}} \frac{\left\|f^{(\alpha)}\right\|_{K}}{\rho^{|\alpha|} M_{|\alpha|}},
$$

where $\|u\|_{K}:=\sup _{x \in K}|u(x)|$ denotes the supremum norm. (In the definition of $\|f\|_{K, \rho}^{M}$ it is not important that $K$ is compact; we will occasionally use $\|f\|_{U, \rho}^{M}$ for open sets $U$.) We define the Denjoy-Carleman class of Beurling type

$$
\mathcal{E}^{(M)}(U):=\left\{f \in C^{\infty}(U): \forall K \subseteq_{c p} U \forall \rho>0:\|f\|_{K, \rho}^{M}<\infty\right\}
$$

and the Denjoy-Carleman class of Roumieu type

$$
\mathcal{E}^{\{M\}}(U):=\left\{f \in C^{\infty}(U): \forall K \subseteq_{c p} U \exists \rho>0:\|f\|_{K, \rho}^{M}<\infty\right\} .
$$

It is convenient to consider also the global Denjoy-Carleman classes

$$
\begin{aligned}
& \mathcal{B}^{(M)}(U):=\left\{f \in C^{\infty}(U): \forall \rho>0:\|f\|_{U, \rho}^{M}<\infty\right\}, \\
& \mathcal{B}^{\{M\}}(U):=\left\{f \in C^{\infty}(U): \exists \rho>0:\|f\|_{U, \rho}^{M}<\infty\right\},
\end{aligned}
$$

as well as the Banach space $\mathcal{B}_{\rho}^{M}(U)=\left\{f \in C^{\infty}(U):\|f\|_{U, \rho}^{M}<\infty\right\}$. We consider the natural locally convex topologies on these spaces, i.e.,

$$
\mathcal{B}^{(M)}(U)=\operatorname{proj}_{n \in \mathbb{N}} \mathcal{B}_{1 / n}^{M}(U), \quad \mathcal{B}^{\{M\}}(U)=\operatorname{ind}_{n \in \mathbb{N}} \mathcal{B}_{n}^{M}(U)
$$

and

$$
\mathcal{E}^{[M]}(U)=\operatorname{proj}_{V \Subset U} \mathcal{B}^{[M]}(V)
$$

Convention 2.1. For notational convenience we use $\mathcal{E}^{[M]}$ as placeholder for either $\mathcal{E}^{(M)}$ or $\mathcal{E}^{\{M\}}$ with the understanding that if the placeholder appears repeatedly in a statement then it must be interpreted by either $\mathcal{E}^{(M)}$ or $\mathcal{E}^{\{M\}}$ at all instances. In an analogous fashion we use $\mathcal{B}^{[M]}, \mathcal{D}^{[M]}$, etc.

The spaces $\mathcal{B}^{(M)}(U)$ and $\mathcal{E}^{(M)}(U)$ are Fréchet spaces, $\mathcal{B}^{\{M\}}(U)$ is a Silva space, and all spaces are nuclear provided that the weight sequence $M$ is derivation-closed, see Section 2.5.

Furthermore, we need the subspaces consisting of functions with compact support: for compact $K \subseteq \mathbb{R}^{n}$ let

$$
\mathcal{D}^{[M]}(K):=\left\{f \in \mathcal{E}^{[M]}\left(\mathbb{R}^{n}\right): \operatorname{supp} f \subseteq K\right\}
$$

with the induced topology. If $U \subseteq \mathbb{R}^{n}$ is open, we set

$$
\mathcal{D}^{[M]}(U):=\operatorname{ind}_{K \subseteq_{c p} U} \mathcal{D}^{[M]}(K) .
$$

We shall see below that $\mathcal{D}^{[M]}(U)$ can be trivial.

For the sequence $M_{k}=k$ !, the Roumieu class $\mathcal{E}^{\{M\}}(U)$ coincides with the class of real analytic functions $C^{\omega}(U)$ and the Beurling class $\mathcal{E}^{(M)}(U)$ consists of the restrictions to $U$ of the entire functions $\mathcal{H}\left(\mathbb{C}^{n}\right)$ on $\mathbb{C}^{n}$. This follows from the Cauchy 
estimates. In general the sequence $M$ describes a deviation from the Cauchy estimates.

2.2. Weight sequences. It is convenient to require some basic mild regularity conditions for the sequence $M$. Most importantly we assume that $M$ is log-convex, that is $\left(\log M_{k}\right)_{k}$ is a convex sequence. ${ }^{2}$ Equivalently, the associated sequence $\mu$ with

$$
\mu_{k}:=\frac{M_{k}}{M_{k-1}}, \quad k \geq 1,
$$

is increasing. We also assume $\mu_{0}:=1 \leq \mu_{1}$.

Definition 2.2. We call a positive log-convex sequence $M=\left(M_{k}\right)$ with $M_{0}=1 \leq$ $M_{1}$ and $M_{k}^{1 / k} \rightarrow \infty$ a weight sequence.

Log-convexity of the sequence $m$ defined by

$$
m_{k}:=\frac{M_{k}}{k !}
$$

is a stronger useful property; it means that $\mu^{*}$ with $\mu_{0}^{*}:=1$ and

$$
\mu_{k}^{*}:=\frac{m_{k}}{m_{k-1}}=\frac{\mu_{k}}{k}, \quad k \geq 1,
$$

is increasing. A weight sequence $M$ with this property is said to be strongly logconvex.

Lemma 2.3. A positive log-convex sequence $M$ with $M_{0}=1 \leq M_{1}$ has the following properties:

(1) $M_{k}^{1 / k} \leq \mu_{k}$ for all $k \geq 1$.

(2) The sequences $\left(M_{k}\right)$ and $\left(M_{k}^{1 / k}\right)$ are increasing.

(3) $M_{j} M_{k} \leq M_{j+k}$ for all $j, k \in \mathbb{N}$.

(4) $M_{k}^{1 / k} \rightarrow \infty$ if and only if $\mu_{k} \rightarrow \infty$.

Proof. By assumption, we have $1 \leq \mu_{1} \leq \mu_{2} \leq \cdots$ and thus $M_{k}^{1 / k}=$ $\left(\mu_{1} \mu_{2} \cdots \mu_{k}\right)^{1 / k} \leq \mu_{k}$, that is (1). An easy computation shows that $M_{k}^{1 / k} \leq$ $M_{k+1}^{1 /(k+1)}$ is equivalent to $M_{k}^{1 / k} \leq \mu_{k+1}$. So (2) follows. To see (3) observe that $M_{j} M_{k} \leq\left(\mu_{1} \cdots \mu_{j}\right)\left(\mu_{1} \cdots \mu_{k}\right) \leq \mu_{1} \cdots \mu_{j+k}=M_{j+k}$. Let us check (4). That $M_{k}^{1 / k} \rightarrow \infty$ implies $\mu_{k} \rightarrow \infty$ follows from (1). If $\mu_{k} \rightarrow \infty$, then for each positive integer $n$ there is $k_{n}$ such that $\mu_{k} \geq n$ for all $k \geq k_{n}$. Then

$$
M_{2 k_{n}}=M_{k_{n}} \mu_{k_{n}+1} \cdots \mu_{2 k_{n}} \geq n^{k_{n}}
$$

and thus $M_{2 k_{n}}^{1 /\left(2 k_{n}\right)} \geq \sqrt{n}$ for all $n$. So $M_{k}^{1 / k} \rightarrow \infty$, since $M_{k}^{1 / k}$ is increasing.

\footnotetext{
${ }^{2}$ Generally, this assumption can be made without loss of generality. Indeed, if $M$ is a positive sequence, then $\mathcal{E}^{\{M\}}(U)=\mathcal{E}^{\{\underline{M}\}}(U)$ and $\mathcal{E}^{(M)}(U)=\mathcal{E}^{(\underline{M})}(U)$, where $\underline{M}$ is the log-convex minorant of $M$, provided that the classes contain the real analytic class, respectively. This follows from the Cartan-Gorny inequality, see [41, 26] and [74, Theorem 2.15].
} 
2.3. Characteristic functions. Every Roumieu class contains functions whose derivatives max out the defining bounds.

Lemma 2.4. If $M=\left(M_{k}\right)$ is a weight sequence, then there exists $f \in \mathcal{B}^{\{M\}}(\mathbb{R}, \mathbb{C})$ such that $f^{(k)}(0)=i^{k} a_{k}$ with $a_{k} \geq M_{k}$ for all $k \in \mathbb{N}$ and $i=\sqrt{-1}$.

Proof. The following construction is due to [3], see also [88, Theorem 1]. Since $\mu_{k}$ is increasing, we have

$$
\mu_{k+1}^{j-k} \leq \frac{M_{j}}{M_{k}}, \quad \text { for all }(j, k) \in \mathbb{N}^{2} .
$$

Then the function

$$
f(x):=\sum_{k=0}^{\infty} \frac{M_{k}}{\left(2 \mu_{k+1}\right)^{k}} e^{2 i \mu_{k+1} x}
$$

has the required properties. Indeed, $j$-fold term-wise differentiation yields

$$
\left|\sum_{k=0}^{\infty} \frac{M_{k}}{\left(2 \mu_{k+1}\right)^{k}}\left(2 i \mu_{k+1}\right)^{j} e^{2 i \mu_{k+1} x}\right| \leq M_{j} \sum_{k=0}^{\infty} 2^{j-k}=2^{j+1} M_{j}
$$

whence the series is uniformly convergent and $f \in \mathcal{B}^{\{M\}}(\mathbb{R}, \mathbb{C})$. Moreover,

$$
\frac{f^{(j)}(0)}{i^{j}}=\sum_{k=0}^{\infty} \frac{M_{k}}{\left(2 \mu_{k+1}\right)^{k-j}} \geq M_{j} .
$$

The lemma is proved.

2.4. Inclusion relations. Let $M=\left(M_{k}\right)$ and $N=\left(N_{k}\right)$ be positive sequences. It follows from the definition that the inclusion $\mathcal{E}^{[M]}(U) \subseteq \mathcal{E}^{[N]}(U)$ holds for all open $U \subseteq \mathbb{R}^{n}$ and all $n \geq 1$, provided that the positive sequences $M$ und $N$ satisfy

$$
\sup _{k \in \mathbb{N}}\left(\frac{M_{k}}{N_{k}}\right)^{1 / k}<\infty .
$$

In that case we write $M \preccurlyeq N$. Notice that the inclusion $\mathcal{E}^{(M)}(U) \subseteq \mathcal{E}^{\{M\}}(U)$ is trivially true. It is also clear from the definition that

$$
\lim _{k \rightarrow \infty}\left(\frac{M_{k}}{N_{k}}\right)^{1 / k}=0
$$

implies the inclusion $\mathcal{E}^{\{M\}}(U) \subseteq \mathcal{E}^{(N)}(U)$ for all open $U \subseteq \mathbb{R}^{n}, n \geq 1$. We abbreviate this relation by $M \triangleleft N$.

Lemma 2.5. Let $M$ be a weight sequence and $N$ a positive sequence. Then:

(1) The inclusion $\mathcal{E}^{[M]}(U) \subseteq \mathcal{E}^{[N]}(U)$ for all open $U \subseteq \mathbb{R}^{n}, n \geq 1$, is equivalent to $M \preccurlyeq N$.

(2) The inclusion $\mathcal{E}^{\{M\}}(U) \subseteq \mathcal{E}^{(N)}(U)$ for all open $U \subseteq \mathbb{R}^{n}, n \geq 1$, is equivalent to $M \triangleleft N$.

(3) The inclusion $\mathcal{E}^{(M)}(U) \subseteq \mathcal{E}^{\{N\}}(U)$ for all open $U \subseteq \mathbb{R}^{n}, n \geq 1$, is equivalent to $M \preccurlyeq N$.

Actually, for the necessity of $M \preccurlyeq N$, respectively $M \triangleleft N$, it is enough to have the respective inclusion relation for some non-empty open subset $U$ of $\mathbb{R}$. 
If the smaller space is of Roumieu type, then the necessity of the various conditions follows easily from Lemma 2.4. That $\mathcal{E}^{(M)}(\mathbb{R}) \subseteq \mathcal{E}^{(N)}(\mathbb{R})$ and $\mathcal{E}^{(M)}(\mathbb{R}) \subseteq$ $\mathcal{E}^{\{N\}}(\mathbb{R})$ imply $M \preccurlyeq N$, respectively, can be shown by an argument of [21] based on the closed graph theorem.

Let $M$ and $N$ be weight sequences. The lemma implies that $\mathcal{E}^{[M]}=\mathcal{E}^{[N]}$ if and only if $M \preccurlyeq N \preccurlyeq M$. In that case we say that the weight sequences $M$ and $N$ are equivalent. (Here (and below) $\mathcal{E}^{[M]} \subseteq \mathcal{E}^{[N]}$ means that the inclusion $\mathcal{E}^{[M]}(U) \subseteq \mathcal{E}^{[N]}(U)$ holds for all open subsets $U \subseteq \mathbb{R}^{n}, n \geq 1$.)

In view of $C^{\omega}(U) \cong \mathcal{E}^{\left\{(k !)_{k}\right\}}(U)$ and $\mathcal{H}\left(\mathbb{C}^{n}\right) \cong \mathcal{E}^{\left((k !)_{k}\right)}(\bar{U})$ one easily deduces the following corollary.

Corollary 2.6. Let $M$ be a positive sequence. Then:

(1) The inclusions $C^{\omega}(U) \subseteq \mathcal{E}^{\{M\}}(U)$ and $\mathcal{H}\left(\mathbb{C}^{n}\right) \subseteq \mathcal{E}^{(M)}(U)$ for all open $U \subseteq \mathbb{R}^{n}, n \geq 1$, are both equivalent to the condition

$$
\liminf _{k \rightarrow \infty} m_{k}^{1 / k}>0
$$

(2) The inclusion $C^{\omega}(U) \subseteq \mathcal{E}^{(M)}(U)$ for all open $U \subseteq \mathbb{R}^{n}, n \geq 1$, is equivalent to the condition

$$
\lim _{k \rightarrow \infty} m_{k}^{1 / k}=\infty
$$

In this case the inclusion $C^{\omega}(U) \subseteq \mathcal{E}^{(M)}(U)$ is strict.

2.5. Stability properties. Under suitable assumptions on the weight sequence, Denjoy-Carleman classes are stable under basic operations of smooth analysis. Let $M=\left(M_{k}\right)$ be a weight sequence.

Pointwise multiplication: Let $U \subseteq \mathbb{R}^{n}$ be open. Then $\mathcal{E}^{[M]}(U)$ forms a ring with respect to pointwise multiplication of functions. This follows from the Leibniz rule and Lemma 2.3(3).

Analytic change of variables: Suppose that $C^{\omega}(U) \subseteq \mathcal{E}^{[M]}(U)$ and $\varphi$ : $V \rightarrow U$ is real analytic, $V \subseteq \mathbb{R}^{m}$ open. Then the pullback $\varphi^{*}: \mathcal{E}^{[M]}(U) \rightarrow$ $\mathcal{E}^{[M]}(V), \varphi^{*}(f)=f \circ \varphi$, is well-defined; cf. [40]. Hence one can consider $\mathcal{E}^{[M]}$-functions on real analytic manifolds.

For the following properties the weight sequence $M$ must satisfy additional conditions.

Stability under differentiation: $\mathcal{E}^{[M]}(U)$ is stable under differentiation (i.e. $\partial^{\alpha} \mathcal{E}^{[M]}(U) \subseteq \mathcal{E}^{[M]}(U)$ for all $\alpha \in \mathbb{N}^{n}$ ) if and only if

$$
\sup _{k \geq 1}\left(\frac{M_{k+1}}{M_{k}}\right)^{1 / k}<\infty \text {. }
$$

In this case we say that $M$ is derivation-closed. It is easy to see that (2.1) implies that there is a constant $C \geq 1$ such that $M_{k} \leq C^{k^{2}}$ for all $k$. Actually, also the converse implication holds if $M$ is log-convex, see [58].

Stability under composition: $\mathcal{E}^{[M]}$ is stable under composition (i.e., $\mathcal{E}^{[M]}(U) \circ \mathcal{E}^{[M]}(V, U) \subseteq \mathcal{E}^{[M]}(V)$ for all open sets $U \subseteq \mathbb{R}^{n}$ and $\left.V \subseteq \mathbb{R}^{m}\right)$ provided that

$$
M^{\circ} \preccurlyeq M,
$$


where $m_{k}^{\circ}:=\max \left\{m_{j} m_{\alpha_{1}} \cdots m_{\alpha_{j}}: \alpha_{i} \in \mathbb{N}_{>0}, \alpha_{1}+\cdots+\alpha_{j}=k\right\}$. This follows easily from Faà di Bruno's formula; cf. [74]. ${ }^{3}$ We call (2.2) the $(F d B)$-property.

Remark 2.7. The (FdB)-property is not easy to check, but it follows from several conditions that are easier to handle. If $M$ is a positive sequence, then each of the following conditions implies that $M$ has the (FdB)-property:

(1) $m=\left(m_{k}\right)$ is log-convex (i.e. $M$ is strongly log-convex).

(2) $m_{j} m_{k} \leq m_{1} m_{j+k-1}$ for all $j, k \geq 1$.

(3) $M$ is derivation-closed and the sequence $m_{k}^{1 / k}$ is almost increasing, i.e., there exists $C>0$ such that $m_{j}^{1 / j} \leq C m_{k}^{1 / k}$ for all $j \leq k$.

Conversely, if $M$ is a weight sequence having the (FdB)-property, then $m_{k}^{1 / k}$ is almost increasing; cf. [74]. See [74, 3.6] for an example of a weight sequence $M$ such that $\mathcal{E}^{[M]}$ is stable under composition, but there is no strongly log-convex sequence $N$ that is equivalent to $M$.

Inverse mappings: For any $\mathcal{E}^{[M]}$-mapping $f: U \rightarrow V$, where $U \subseteq \mathbb{R}^{m}$ and $V \in \mathbb{R}^{n}$ are open, such that $f^{\prime}\left(x_{0}\right) \in L\left(\mathbb{R}^{m}, \mathbb{R}^{n}\right)$ is invertible at $x_{0} \in U$ there exist neighborhoods $x_{0} \in U_{0} \subseteq U$ and $f\left(x_{0}\right) \in V_{0} \subseteq V$ and a $\mathcal{E}^{[M]_{-}}$ mapping $g: V_{0} \rightarrow U_{0}$ such that $f \circ g=\operatorname{id}_{V_{0}}$, provided that $C^{\omega} \subseteq \mathcal{E}^{[M]}, M$ is derivation-closed, and $m_{k}^{1 / k}$ is almost increasing. Under these assumptions we evidently also have the implicit mapping theorem in $\mathcal{E}^{[M]}$.

Solving ODEs: For any $\mathcal{E}^{[M]}$-mapping $f: \mathbb{R} \times \mathbb{R}^{n} \rightarrow \mathbb{R}^{n}$ the solution of the initial value problem $x^{\prime}=f(t, x), x(0)=x_{0}$, is of class $\mathcal{E}^{[M]}$, where it exists, provided that $C^{\omega} \subseteq \mathcal{E}^{[M]}, M$ is derivation-closed, and $m_{k}^{1 / k}$ is almost increasing.

It was proved in [75] that, given that $C^{\omega} \subseteq \mathcal{E}^{[M]}$ and $M$ is derivation-closed, the condition that $m_{k}^{1 / k}$ is almost increasing is also necessary for stability under composition, inverse mappings, and solving ODEs, respectively. As a consequence $\mathcal{E}^{[M]}$ is inverse-closed, that is $1 / f \in \mathcal{E}^{[M]}(U)$ if $f \in \mathcal{E}^{[M]}(U)$ is non-vanishing.

2.6. Moderate growth. Let us finish this section by briefly discussing another property which often plays a decisive role. We say that a weight sequence $M$ has moderate growth if

$$
\exists C>0 \forall j, k \in \mathbb{N}: M_{j+k} \leq C^{j+k} M_{j} M_{k} .
$$

Evidently, (2.3) entails (2.1). It is not hard to see that (2.3) is equivalent to $\mu_{k+1} \lesssim M_{k}^{1 / k}$ and in turn to $\mu_{2 k} \lesssim \mu_{k}$; cf. [76, Lemma 2.2]. In terms of the spaces $\mathcal{E}^{[M]}$, moderate growth of $M$ is equivalent to separativity [59], validity of the exponential law [48, 49,50], and stability under ultradifferential operators [47], respectively. Note that (2.3) holds for $M$ if and only if it holds for $m$.

The moderate growth condition is rather restrictive. Indeed, it implies that there is a constant $C>0$ such that $\mu_{2^{j}} \leq C \mu_{2^{j-1}}$ for all $j$, and thus, if $2^{j} \leq k<2^{j+1}$,

$$
\mu_{k} \leq \mu_{2^{j+1}} \leq C \mu_{2^{j}} \leq C^{j+1} \leq C^{j+1} \frac{k^{s}}{2^{j s}}
$$

\footnotetext{
${ }^{3}$ It was shown in [74] that $(2.2)$ is also necessary for the stability under composition of $\mathcal{E}^{[M]}$ provided that the class is stable by differentiation; this is based on the characterization of inverseclosedness and stability of $\mathcal{E}^{[M]}$ under superposition by entire functions due to [81, 21].
} 
for any $s \geq 1$. If we choose $s \geq 1$ such that the sequence $\left(C^{j+1} / 2^{j s}\right)_{j}$ is bounded, we find that $M \preccurlyeq G^{s}$, where $G_{k}^{s}:=k !^{s}$.

Example 2.8. For $s \geq 1$ the sequence $G^{s}=\left(G_{k}^{s}\right)$ is a strongly log-convex weight sequences of moderate growth. It is called the Gevrey sequence of index $s$. We have $\mathcal{E}^{\left\{G^{1}\right\}}=C^{\omega}$. For $s>1$ a typical function in $\mathcal{E}^{\left\{G^{s}\right\}}(\mathbb{R})$ is $t \mapsto \exp \left(-1 / t^{1 /(s-1)}\right)$. It is easy to check that $\left(k^{s k}\right)_{k}$ is an equivalent weight sequence.

\section{The Borel map and the Denjoy-Carleman theorem}

Coming back to Question 1.1 we shall now discuss our problem for the singleton $\{0\}$. Suppose that $M$ is a weight sequence and $f \in \mathcal{E}^{[M]}(U)$, where $U$ is an open connected neighborhood of $0 \in \mathbb{R}^{n}$. Then the jet $F:=j_{\{0\}}^{\infty} f=\left(f^{(\alpha)}(0)\right)_{\alpha \in \mathbb{N} n}$ obviously satisfies

$$
|F|_{\rho}^{M}:=\sup _{\alpha \in \mathbb{N}^{n}} \frac{\left|F^{\alpha}\right|}{\rho^{|\alpha|} M_{|\alpha|}}<\infty
$$

for some $\rho>0$ or for all $\rho>0$, depending on whether we consider the Roumieu case $\mathcal{E}^{\{M\}}$ or the Beurling case $\mathcal{E}^{(M)}$. We define

$$
\begin{aligned}
\Lambda_{n}^{\{M\}}: & =\left\{a \in \mathbb{C}^{\mathbb{N}^{n}}: \exists \rho>0:|a|_{\rho}^{M}<\infty\right\}, \\
\Lambda_{n}^{(M)} & :=\left\{a \in \mathbb{C}^{\mathbb{N}^{n}}: \forall \rho>0:|a|_{\rho}^{M}<\infty\right\},
\end{aligned}
$$

and equip them with their natural locally convex topology. In accordance with Convention 2.1, we use $\Lambda_{n}^{[M]}$ for both of them and just write $\Lambda^{[M]}$ if the dimension $n$ is clear from the context. Now the (restriction of the) Borel map

$$
j_{\{0\}}^{\infty}: \mathcal{E}^{[M]}(U) \rightarrow \Lambda^{[M]}
$$

is well-defined. We may specify Question 1.1 in this setting and ask:

When is the Borel mapping (3.2) surjective or injective?

We start with discussing injectivity.

3.1. Quasianalyticity. Let $M$ be a weight sequence. We say that the class $\mathcal{E}^{[M]}$ is quasianalytic if for all open connected neighborhoods $U$ of $0 \in \mathbb{R}^{n}$ the Borel map $j_{\{0\}}^{\infty}: \mathcal{E}^{[M]}(U) \rightarrow \Lambda^{[M]}$ is injective. The class $\mathcal{E}^{[M]}$ is called non-quasianalytic if it is not quasianalytic. In that case it is easy to construct non-trivial functions with compact support (so-called bump functions) in the class, since $\mathcal{E}^{[M]}$ is stable by multiplication. $^{4}$

Remark 3.1. There is nothing special about the origin. It is easy to see that $\mathcal{E}^{[M]}$ is quasianalytic if and only if $j_{\{a\}}^{\infty}: \mathcal{E}^{[M]}(V) \rightarrow \Lambda^{[M]}$ is injective, where $V \subseteq \mathbb{R}^{n}$ is any open connected neighborhood of an arbitrary point $a$.

Note that it suffices to check in dimension one whether a class $\mathcal{E}^{[M]}$ is quasianalytic or not. Indeed, if there exists a bump function $f$ in dimension one. Then $f \otimes \cdots \otimes f(n$ times $)$ is a bump function in dimension $n$. And, if a function $f$ defined in a neighborhood of $0 \in \mathbb{R}^{n}$ satisfies $j_{\{0\}}^{\infty} f=0$, then $j_{\{0\}}^{\infty}(f \circ \ell)=0$ for all lines $\ell$ through 0 .

\footnotetext{
${ }^{4}$ The study of quasianalytic classes started at the end of the nineteenth century with the work of Émile Borel who found examples of non-trivial sets $\mathcal{C}$ of smooth functions on $\mathbb{R}$ containing nowhere analytic elements such that all members $f$ of $\mathcal{C}$ have the unique continuation property $\left(\forall k \in \mathbb{N}: f^{(k)}(0)=0\right) \Longrightarrow f=0$.
} 
In a communication [42] to the Société Mathématique de France Jacques Hadamard asked whether quasianalyticity can be characterized in terms of a growth condition on the iterated derivatives. This was essentially confirmed a decade later by [32] and [24, 23] and became known as the Denjoy-Carleman theorem. We shall give a proof based on the approach of [31] and [44, Theorem 1.3.8].

\subsection{The size of a function near points of flatness.}

Lemma 3.2. Let $M=\left(M_{k}\right)$ be a weight sequence. Let $I=(-r, r) \subseteq \mathbb{R}$ for some $r>0$. Let $f \in C^{\infty}(I)$ be such that $f(t)=0$ for all $t \leq 0$ and $\left\|f^{(k)}\right\|_{I} \leq M_{k}$ for $0 \leq k \leq n$. Then, for all $1 \leq \ell \leq n$ and $0 \leq t \leq \min \left\{r, \frac{1}{4} \sum_{k=\ell}^{n} \frac{1}{\mu_{k}}\right\}$,

$$
|f(t)| \leq\left(\frac{2 t}{\sum_{k=\ell}^{n} \frac{1}{\mu_{k}}}\right)^{\ell} .
$$

Proof. Cf. [14] which is based on [3] and [31]. Fix $\ell$ with $1 \leq \ell \leq n$ and $t=t_{\ell} \in$ $(0, r)$. Let $t_{\ell+1}>t_{\ell+2}>\cdots>t_{n+1}=0$ be such that the interval $I_{k}:=\left[t_{k+1}, t_{k}\right]$ has length

$$
\left|I_{k}\right|=\frac{a}{\mu_{k}}, \quad \text { where } \quad a:=\frac{t_{\ell}}{\sum_{k=\ell}^{n} \frac{1}{\mu_{k}}} .
$$

Additionally, we set $I_{n+1}:=(-r, 0]$. We define $F(s, k)=\left\|f^{(s)}\right\|_{I_{k}}$ and claim

$$
F(s, k) \leq(2 a)^{k-s} M_{s} \quad \text { for } \ell \leq k \leq n+1 \text { and } 0 \leq s \leq k .
$$

The claim holds for $s=k$ and for $s \leq k=n+1$ by assumption, and the fundamental theorem of calculus gives the bound

$$
F(s, k) \leq F(s, k+1)+\left|I_{k}\right| F(s+1, k) .
$$

Let $0 \leq s<k \leq n$. If we assume by induction, that (3.4) is true for $(s, k+1)$ and $(s+1, k)$, then

$$
F(s, k) \leq(2 a)^{k+1-s} M_{s}+\frac{a}{\mu_{k}}(2 a)^{k-s-1} M_{s+1} \leq(2 a)^{k-s} M_{s}\left(2 a+\frac{1}{2}\right),
$$

since $M_{s+1}=M_{s} \mu_{s+1} \leq M_{s} \mu_{k}$. Provided that $2 a \leq 1 / 2$, claim (3.4) is proved by induction. In particular, for $t=t_{\ell} \in I_{\ell}$ and $s=0$, we obtain $|f(t)| \leq F(0, \ell) \leq(2 a)^{\ell}$ which is (3.3).

As an immediate consequence we observe that the divergence of the series $\sum_{k} \frac{1}{\mu_{k}}$ is a sufficient condition for the injectivity of $j_{\{0\}}^{\infty}: \mathcal{E}^{\{M\}}(\mathbb{R}) \rightarrow \Lambda^{\{M\}}$. To see that it is also necessary we aim to construct a non-trivial function with compact support in $\mathcal{E}^{\{M\}}(\mathbb{R})$ provided that the series converges.

3.3. Special bump functions. For $a>0$ let us consider the step function $H_{a}:=$ $\frac{1}{a} \mathbf{1}_{(0, a)}$. If $f$ is a continuous function on $\mathbb{R}$, then

$$
f * H_{a}(x)=\frac{1}{a} \int_{0}^{a} f(x-t) d t=\frac{1}{a} \int_{x-a}^{x} f(t) d t
$$

is $C^{1}$ with derivative

$$
\left(f * H_{a}\right)^{\prime}(x)=\frac{f(x)-f(x-a)}{a} .
$$

Hence $f * H_{a}$ is $C^{k+1}$ provided that $f$ is $C^{k}$. 
Lemma 3.3. Let $\left(a_{k}\right)$ be a decreasing positive sequence with $a:=\sum_{k=0}^{\infty} a_{k}<\infty$. Then $f_{k}:=H_{a_{0}} * \cdots * H_{a_{k}}$ is of class $C^{k-1}$ and has support in $[0, a]$. The sequence $\left(f_{k}\right)$ converges to a $C^{\infty}$-function $f$ with support in $[0, a]$ such that $\int f d x=1$ and

$$
\left|f^{(k)}(x)\right| \leq \frac{1}{2} \int\left|f^{(k+1)}(x)\right| d x \leq \frac{2^{k}}{a_{0} a_{1} \cdots a_{k}},
$$

for all $k \in \mathbb{N}$ and $x \in \mathbb{R}$.

Proof. Cf. [44, Theorem 1.3.5]. It is easy to see that $f_{1}$ is the piecewise linear function which vanishes outside $\left[0, a_{0}+a_{1}\right]$ has slope $\left(a_{0} a_{1}\right)^{-1}$ in $\left[0, a_{1}\right]$, is constant in $\left[a_{1}, a_{0}\right]$ and decreases linearly to 0 in $\left[a_{0}, a_{0}+a_{1}\right]$. In particular, $f_{1}$ is continuous.

It follows that $f_{k}$ is $C^{k-1}$ with support in $\left[0, a_{0}+a_{1}+\cdots+a_{k}\right]$. Writing $\left(\tau_{a} f\right)(x)=$ $f(x-a)$ for the translation operator, we have

$$
f_{k}^{\prime}(x)=H_{a_{0}}^{\prime} * H_{a_{1}} * \cdots * H_{a_{k}}(x)=\frac{1}{a_{0}}\left(1-\tau_{a_{0}}\right) H_{a_{1}} * \cdots * H_{a_{k}}(x)
$$

and iterating we find

$$
f_{k}^{(j)}=\prod_{i=0}^{j-1} \frac{1}{a_{i}}\left(1-\tau_{a_{i}}\right) H_{a_{j}} * \cdots * H_{a_{k}}, \quad \text { for } j \leq k-1 .
$$

Since $\int H_{a_{i}} d x=1$ for all $i$, it follows that

$$
\left|f_{k}^{(j)}\right| \leq \frac{2^{j}}{a_{0} a_{1} \cdots a_{j}} \quad \text { and } \quad \int\left|f_{k}^{(j)}\right| d x \leq \frac{2^{j}}{a_{0} a_{1} \cdots a_{j-1}},
$$

where we use $|u * v| \leq \sup |u| \int|v| d x$ and $\int u * v d x=\int u d x \int v d x$. We have

$$
\begin{aligned}
\left|f_{k+\ell}(x)-f_{\ell}(x)\right| & =\left|f_{\ell} * H_{a_{\ell+1}} * \cdots * H_{a_{\ell+k}}(x)-f_{\ell}(x)\right| \\
& =\left|\int\left(f_{\ell}(x-y)-f_{\ell}(x)\right) H_{a_{\ell+1}} * \cdots * H_{a_{\ell+k}}(y) d y\right| \\
& \leq\left(a_{\ell+1}+\cdots+a_{\ell+k}\right) \sup \left|f_{\ell}^{\prime}\right| \\
& \leq 2 \frac{a_{\ell+1}+\cdots+a_{\ell+k}}{a_{0} a_{1}},
\end{aligned}
$$

since $H_{a_{\ell+1}} * \cdots * H_{a_{\ell+k}}$ has support in $\left[0, a_{\ell+1}+\cdots+a_{\ell+k}\right]$. It follows that $f_{k}$ has a uniform limit $f$. Similarly, one sees that all derivatives of $f_{k}$ have uniform limits. Hence $f$ is $C^{\infty}$ and the lemma follows easily.

3.4. The Denjoy-Carleman theorem. We are ready to characterize quasianalyticity in the Roumieu case. In order to also include the Beurling case we first treat the following lemma. This lemma will be very useful for reducing the Beurling to the Roumieu case at several occasions.

Lemma 3.4 ([27, Lemma 16]). Let $\left(\alpha_{k}\right)_{k \geq 1},\left(\beta_{k}\right)_{k \geq 1}$, and $\left(\gamma_{k}\right)_{k \geq 1}$ be sequences with the following properties:

(1) $\alpha_{k} \geq 0$ and $\sum_{k} \alpha_{k}<\infty$.

(2) $\beta_{k}>0$ and $\beta_{k} \rightarrow 0$.

(3) $\gamma_{k}>0$ and $\gamma_{k} \searrow 0$.

Then there exists a positive sequence $\left(\lambda_{k}\right)_{k \geq 1}$ such that $\lambda_{k} \nearrow \infty$ and

(1) $\sum_{j \geq k} \lambda_{j} \alpha_{j} \leq 8 \lambda_{k} \sum_{j \geq k} \alpha_{j}$ for all $k \geq 1$,

(2) $\lambda_{k} \beta_{k} \rightarrow 0$,

(3) $\lambda_{k} \gamma_{k}$ is decreasing. 
Proof. We find a strictly increasing sequence $\left(j_{p}\right)_{p \geq 1}$ of integers with $j_{1}=1$ and

$$
\sum_{j \geq j_{p+1}} \alpha_{j} \leq \frac{1}{4} \sum_{j \geq j_{p}} \alpha_{j}, \quad \beta_{j} \leq \frac{1}{4^{p}} \text { if } j \geq j_{p+1}, \quad \gamma_{j_{p+1}} \leq \frac{1}{4} \gamma_{j_{p}}
$$

for all $p \geq 1$. Then $2^{p+1} \gamma_{j_{p+1}}<2^{p} \gamma_{j_{p}}<2^{p+1} \gamma_{j_{p}}$. Let $k_{p}$ be the largest integer $k$ satisfying $j_{p} \leq k<j_{p+1}$ and $2^{p} \gamma_{j_{p}} \leq 2^{p+1} \gamma_{k}$. Define $\left(\lambda_{k}\right)_{k \geq 1}$ by setting

$$
\lambda_{k}:= \begin{cases}2^{p} \frac{\gamma_{j_{p}}}{\gamma_{k}} & \text { if } j_{p} \leq k \leq k_{p}, \\ 2^{p+1} & \text { if } k_{p}<k \leq j_{p+1} .\end{cases}
$$

It is clear that $\lambda_{k} \nearrow \infty$ and that $\lambda_{k} \gamma_{k}$ is decreasing. If $j_{p+1} \leq k \leq j_{p+2}$, then $2^{p+1} \leq \lambda_{k} \leq 2^{p+2}$ and hence $\lambda_{k} \beta_{k} \leq 2^{-p+2}$, whence $\lambda_{k} \beta_{k} \rightarrow 0$. Finally,

$$
\begin{aligned}
\sum_{j \geq j_{p+1}} \lambda_{j} \alpha_{j}=\sum_{n=0}^{\infty} \sum_{j=j_{p+1+n}}^{j_{p+2+n}-1} \lambda_{j} \alpha_{j} & \leq \sum_{n=0}^{\infty} 2^{p+2+n} \sum_{j=j_{p+1+n}}^{j_{p+2+n}-1} \alpha_{j} \\
& \leq \sum_{n=0}^{\infty} 2^{p+2+n} \frac{1}{4^{n}} \sum_{j \geq j_{p+1}} \alpha_{j}=2^{p+3} \sum_{j \geq j_{p+1}} \alpha_{j} .
\end{aligned}
$$

For $k \geq 1$ take $p$ such that $j_{p} \leq k<j_{p+1}$. Then

$$
\begin{aligned}
\sum_{j \geq k} \lambda_{j} \alpha_{j} & =\sum_{j=k}^{j_{p+1}-1} \lambda_{j} \alpha_{j}+\sum_{j \geq j_{p+1}} \lambda_{j} \alpha_{j} \\
& \leq 2^{p+1} \sum_{j=k}^{j_{p+1}-1} \alpha_{j}+2^{p+3} \sum_{j \geq j_{p+1}} \alpha_{j} \leq 8 \cdot 2^{p} \sum_{j \geq k} \alpha_{j} \leq 8 \lambda_{k} \sum_{j \geq k} \alpha_{j} .
\end{aligned}
$$

The proof is complete.

Corollary 3.5. Let $\left(\alpha_{k}\right)$ be a decreasing positive sequence with $\sum_{k} a_{k}<\infty$. There exists a decreasing positive sequence $\left(\beta_{k}\right)$ such that $\sum_{k} \beta_{k}<\infty$ and $\beta_{k} / \alpha_{k} \nearrow \infty$.

Proof. Apply Lemma 3.4 to $\alpha_{k}=\beta_{k}=\gamma_{k}$. Then $\beta_{k}:=\lambda_{k} \alpha_{k}$ has the required properties.

Theorem 3.6 (Denjoy-Carleman theorem). Let $M=\left(M_{k}\right)$ be a weight sequence. Then the following conditions are equivalent:

(1) $\mathcal{E}^{\{M\}}$ is quasianalytic.

(2) $\mathcal{E}^{(M)}$ is quasianalytic.

(3) $\sum_{k} \frac{1}{\mu_{k}}=\infty$.

Proof. The equivalence of (1) and (3) is an easy consequence of Lemma 3.2 and Lemma 3.3 (with $a_{k}=\mu_{k}^{-1}$ ).

If $\mathcal{E}^{(M)}$ contains non-trivial functions of compact support then so does $\mathcal{E}^{\{M\}}$, by the trivial inclusion $\mathcal{E}^{(M)} \subseteq \mathcal{E}^{\{M\}}$, which shows $(3) \Rightarrow(2)$ since we already have the equivalence of (1) and (3).

In order to show that (2) implies (3) suppose that $\sum_{k} \frac{1}{\mu_{k}}<\infty$. By Corollary 3.5, we find an increasing sequence $\left(\nu_{k}\right)$ such that $\sum_{k} \frac{1}{\nu_{k}}<\infty$ and $\frac{\mu_{k}}{\nu_{k}} \nearrow \infty$. So the sequence $\frac{M_{k}}{N_{k}}$ is log-convex and thus $\left(\frac{M_{k}}{N_{k}}\right)^{1 / k} \rightarrow \infty$ since $\frac{\mu_{k}}{\nu_{k}} \rightarrow \infty$ (cf. Lemma 2.3(4)). Then $N=\left(N_{k}\right)$ is a weight sequence satisfying $N \triangleleft M$ and thus $\mathcal{E}^{\{N\}} \subseteq \mathcal{E}^{(M)}$. Since $\sum_{k} \frac{1}{\nu_{k}}<\infty$, we find that $\mathcal{E}^{\{N\}}$ is non-quasianalytic, and hence so is $\mathcal{E}^{(M)}$. 
The Denjoy-Carleman theorem completely characterizes injectivity of the Borel map (3.2). It shows that the following definition makes sense.

Definition 3.7. A weight sequence $M=\left(M_{k}\right)$ is called quasianalytic if the equivalent conditions of Theorem 3.6 hold, and it is called non-quasianalytic otherwise.

Remark 3.8. For later reference note that the proof of the implication $(2) \Rightarrow(3)$ in Theorem 3.6 shows the following: For any non-quasianalytic weight sequence $M$ there exists a non-quasianalytic weight sequence $N$ with $N \triangleleft M$.

Remark 3.9. For a weight sequence $M=\left(M_{k}\right)$, the divergence of the series $\sum_{k} \frac{1}{\mu_{k}}$ is equivalent to the divergence of $\sum_{k} \frac{1}{M_{k}^{1 / k}}$. This follows from $M_{k}^{1 / k} \leq \mu_{k}$ for all $k$ (cf. Lemma 2.3(1)) and Carleman's inequality: If $\left(a_{k}\right)$ is a positive sequence, then

$$
\sum_{n=1}^{\infty}\left(a_{1} a_{2} \cdots a_{n}\right)^{1 / n} \leq e \sum_{k=1}^{\infty} a_{k}
$$

Cf. [44, Lemma 1.3.9].

If $M$ is not necessarily a weight sequence, then one can work with the log-convex minorant

$$
\underline{M}_{k}:=\inf \left\{M_{k}, M_{j}^{\frac{\ell-k}{\ell-j}} M_{\ell}^{\frac{k-j}{\ell-j}}: j<k<\ell\right\}
$$

of $M$ or the increasing minorant

$$
u_{k}:=\inf _{k \geq j} M_{k}^{1 / k}
$$

of $M_{k}^{1 / k}$. In fact, the following are equivalent (see [44, Theorem 1.3.8]):

(1) $\mathcal{E}^{[M]}$ is quasianalytic.

(2) $\sum_{k} \frac{1}{u_{k}}=\infty$.

(3) $\sum_{k} \frac{1}{\frac{M_{k}^{1 / k}}{u_{k}}=\infty}$

(4) $\sum_{k} \frac{1}{\underline{\mu}_{k}}=\infty$.

Example 3.10. (1) The Gevrey sequences $G^{s}, s \geq 1$, are non-quasianalytic if and only if $s>1$.

(2) The Denjoy sequences

$$
Q_{k}^{n, \delta}=k^{k} \prod_{j=1}^{n-1}\left(\log ^{[j]}\left(k+\exp ^{[j]}(1)\right)\right)^{k}\left(\log ^{[n]}\left(k+\exp ^{[n]}(1)\right)\right)^{\delta k}
$$

where $n \in \mathbb{N}_{\geq 1}, 0<\delta \leq 1$, and $f^{[n]}$ is the $n$-fold composite of $f$, are quasianalytic strongly log-convex weight sequences of moderate growth.

3.5. Non-quasianalytic cutoff functions. Before we study surjectivity of the Borel map (3.2) in the next section we show that we may use Lemma 3.3 to construct non-quasianalytic cutoff functions in all dimensions.

Proposition 3.11 ([44, Theorem 1.4.2]). Let $U \subseteq \mathbb{R}^{n}$ be open and $K \subseteq U$ compact. Let $\|\cdot\|$ be any norm on $\mathbb{R}^{n}$. If $d=\inf \left\{\|x-y\|: x \in K, y \in \mathbb{R}^{n} \backslash U\right\}$ and $\left(d_{j}\right)$ is a positive decreasing sequence with $\sum_{j=1}^{\infty} d_{j}<d$, then there exists $\varphi \in C_{c}^{\infty}(U)$ with $0 \leq \varphi \leq 1$ such that $\varphi=1$ on a neighborhood of $K$ and

$$
\left|\varphi^{(k)}(x)\left(v_{1}, \ldots, v_{k}\right)\right| \leq C(n)^{k} \frac{\left\|v_{1}\right\|\left\|v_{2}\right\| \cdots\left\|v_{k}\right\|}{d_{1} d_{2} \cdots d_{k}}, \quad k \geq 1, x \in U .
$$


Proof. Let us first consider the norm $\|x\|=\max _{i}\left|x_{i}\right|$. Let $f$ be the function from Lemma 3.3 (with $a_{j}=d_{j+1}$ ) and set $h(t):=f\left(t+\frac{1}{2} \sum_{j=1}^{\infty} d_{j}\right.$ ). Then $t \in \operatorname{supp} h$ implies $|t| \leq \frac{1}{2} \sum_{j=1}^{\infty} d_{j}$, we have $\int h d t=1$, and

$$
\int\left|h^{(j)}(t)\right| d t \leq \frac{2^{j}}{d_{1} d_{2} \cdots d_{j}} .
$$

Let $\chi(x):=h\left(x_{1}\right) h\left(x_{2}\right) \cdots h\left(x_{n}\right)$ which satisfies $\int \chi d x=1$ and set $\varphi:=\chi * \mathbf{1}_{K_{d / 2}}$, where $K_{d / 2}:=\left\{y \in \mathbb{R}^{n}:\|x-y\| \leq d / 2\right.$ for some $\left.x \in K\right\}$. Then $\varphi \in C_{c}^{\infty}(U)$ is as required, since

$$
\left|\varphi^{(\alpha)}\right| \leq \int\left|\chi^{(\alpha)}(x)\right| d x \leq \frac{2^{|\alpha|}}{d_{1} d_{2} \cdots d_{|\alpha|}}
$$

as $\left(d_{k}\right)$ is decreasing. The statement for arbitrary norms follows from the fact that any two norms on $\mathbb{R}^{n}$ are equivalent with constants only depending on $n$.

Corollary 3.12. Let $M$ be a non-quasianalytic weight sequence. Let $U \subseteq \mathbb{R}^{n}$ be open and $K \subseteq U$ compact. Then there exists a function $\varphi \in \mathcal{E}^{[M]}(U)$ with support contained in $U$ such that $\varphi=1$ in a neighborhood of $K$.

Proof. Let $d:=\operatorname{dist}\left(K, \mathbb{R}^{n} \backslash U\right)^{5}$ and $d_{j}:=\frac{d}{s \mu_{j}}$, where $s:=\sum_{j \geq 1} \frac{1}{\mu_{j}}<\infty$. Then the proposition provides a function $\varphi \in \mathcal{E}^{\{M\}}(U)$ with the desired properties. Using Remark 3.8, we may achieve that $\varphi \in \mathcal{E}^{(M)}(U)$.

That non-quasianalytic functions are "abundant" is witnessed by the following remark.

Remark 3.13. Let $M$ be a non-quasianalytic weight sequence. For each closed subset $A \subseteq \mathbb{R}^{n}$ there exists $f \in \mathcal{B}^{[M]}\left(\mathbb{R}^{n}\right)$ such that $f$ is flat on $A$, i.e., $j_{A}^{\infty} f=0$, and $\left.f\right|_{\mathbb{R}^{n} \backslash A}$ is positive and real analytic. Cf. $[55,56]$.

\section{SurJectivity of the Borel MaP}

Let $M=\left(M_{k}\right)$ be a weight sequence. We shall see that a necessary and sufficient condition for the surjectivity of the Borel map $j_{\{0\}}^{\infty}: \mathcal{E}^{[M]}(\mathbb{R}) \rightarrow \Lambda^{[M]}$ is

$$
\sup _{k} \frac{\mu_{k}}{k} \sum_{j \geq k} \frac{1}{\mu_{j}}<\infty
$$

In the Beurling case the condition even guarantees that the Borel map is split surjective. This section is primarily based on [68].

Definition 4.1. A weight sequence $M$ satisfying (4.1) is said to be strongly nonquasianalytic. ${ }^{6}$

Obviously a strongly non-quasianalytic weight sequence $M$ is non-quasianalytic, and thus $\mathcal{E}^{[M]}$ admits cutoff functions. We will see that (4.1) allows for special cutoff functions that are in a certain sense optimal; see Section 7.

\footnotetext{
${ }^{5}$ The distance $\operatorname{dist}(A, B):=\inf \{|a-b|: a \in A, b \in B\}$ of two subsets of $\mathbb{R}^{n}$ is defined with respect to the Euclidean norm.

${ }^{6}$ Many authors use Komatsu's (M._) notation [47]: log-convexity (M.1), moderate growth (M.2), strong non-quasianalyticity (M.3), derivation closedness (M.2)', and non-quasianalyticity (M.3)'.
} 
Example 4.2. The Gevrey sequences $G^{s}$ are strongly non-quasianalytic provided that $s>1$. Indeed, $\gamma_{k}^{s}=G_{k}^{s} / G_{k-1}^{s}=k^{s}$ and

$$
\sum_{j \geq k} \frac{1}{j^{s}} \leq \int_{k-1}^{\infty} \frac{1}{t^{s}} d t=\frac{1}{s-1} \frac{1}{(k-1)^{s-1}}=\frac{1}{s-1}\left(\frac{k}{k-1}\right)^{s-1} \frac{k}{k^{s}} \leq \frac{2^{s-1}}{s-1} \frac{k}{k^{s}} .
$$

4.1. Necessity. Let us assume throughout this section that $M$ is nonquasianalytic. The quasianalytic case which requires different methods will be discussed in Section 5.

Theorem 4.3. Let $M$ be a non-quasianalytic weight sequence. If the Borel map $j_{\{0\}}^{\infty}: \mathcal{E}^{(M)}(\mathbb{R}) \rightarrow \Lambda^{(M)}$ or $j_{\{0\}}^{\infty}: \mathcal{E}^{\{M\}}(\mathbb{R}) \rightarrow \Lambda^{\{M\}}$ is surjective, then $M$ is strongly non-quasianalytic.

Proof. We give details in the Beurling case and indicate the required changes for the Roumieu case.

We may suppose that $j_{\{0\}}^{\infty}: \mathcal{B}^{(M)}((-1,1)) \rightarrow \Lambda^{(M)}$ is surjective, by multiplication with a suitable cutoff function. Then the open mapping theorem for Fréchet spaces (cf. [64, 24.30]) implies that there exist constants $C, \rho>0$ and functions $f_{k} \in$ $\mathcal{B}^{(M)}((-1,1))$ such that $f_{k}^{(j)}(0)=\delta_{j k}$ and

$$
\left\|f_{k}\right\|_{[-1,1], 1}^{M} \leq \frac{C}{\rho^{k} M_{k}}, \quad k \in \mathbb{N} .
$$

Let $t_{k}:=\inf \left\{t \in[0,1]: f_{k}^{(k)}(t)<\frac{1}{2}\right\}$. Then $f_{k}^{(k)}(t) \geq \frac{1}{2}$ for $t \in\left[0, t_{k}\right]$ and hence $f_{2 k}^{(k)}(t) \geq \frac{t^{k}}{2 k !}$ for $t \in\left[0, t_{2 k}\right]$. In particular, for $t=t_{2 k}$ we find, by (4.2), $\frac{t_{2 k}^{k}}{2 k !} \leq f_{2 k}^{(k)}\left(t_{2 k}\right) \leq \frac{C M_{k}}{\rho^{2 k} M_{2 k}}$ which implies (using that $\mu_{k}$ is increasing)

$$
t_{2 k} \leq \frac{(2 C)^{1 / k}}{\rho^{2}} \frac{k}{\mu_{k}}
$$

We claim that there exists $0<h<\min \left\{\frac{1}{4}\right.$, $\left.\frac{\rho}{4}\right\}$ such that

$$
t_{k} \geq h \sum_{j \geq 2 k} \frac{1}{\mu_{j}} \quad \text { for all sufficiently large } k .
$$

Then (4.3) and (4.4) imply (4.1),

$$
\sum_{j \geq k} \frac{1}{\mu_{j}}=\sum_{j=k}^{4 k-1} \frac{1}{\mu_{j}}+\sum_{j \geq 4 k} \frac{1}{\mu_{j}} \leq \frac{3 k}{\mu_{k}}+\frac{t_{2 k}}{h} \lesssim \frac{k}{\mu_{k}} .
$$

It remains to show (4.4). To this end we apply Lemma 3.2 to the functions

$$
g_{k}(t):= \begin{cases}\left(\frac{C}{\rho^{k}}+1\right)^{-1}\left(f_{k}^{(k)}(t)-1\right) & \text { if } t \geq 0 \\ 0 & \text { if } t<0 .\end{cases}
$$

Indeed, each $g_{k}$ is $C^{\infty}$, satisfies $\left|g_{k}\right| \leq 1$, and for $j \geq 1$ we have

$$
\left|g_{k}^{(j)}\right|=\left(\frac{C}{\rho^{k}}+1\right)^{-1}\left|f_{k}^{(k+j)}\right| \leq\left(\frac{C}{\rho^{k}}+1\right)^{-1} \frac{C}{\rho^{k}} \frac{M_{k+j}}{M_{k}} \leq \mu_{k+1} \cdots \mu_{k+j}=: M_{j}^{+k},
$$

by (4.2). Then $M^{+k}$ (with $M_{0}^{+k}:=1$ ) is a weight sequence and Lemma 3.2 gives

$$
\left|g_{k}(t)\right| \leq\left(\frac{2 t}{\sum_{j \geq 2 k} \frac{1}{\mu_{j}}}\right)^{k}, \quad 0 \leq t \leq \frac{1}{4} \sum_{j \geq 2 k} \frac{1}{\mu_{j}} .
$$


Suppose for contradiction that (4.4) is violated for infinitely many $k$. For those $k$ we obtain

$$
\frac{1}{2} \frac{\rho^{k}}{C+\rho^{k}}=\left|g_{k}\left(t_{k}\right)\right| \leq\left(\frac{2 t_{k}}{\sum_{j \geq 2 k} \frac{1}{\mu_{j}}}\right)^{k} \leq(2 h)^{k},
$$

which is however only possible for finitely many $k$. Thus (4.4) is proved.

Now suppose that $j_{\{0\}}^{\infty}: \mathcal{B}^{\{M\}}((-1,1)) \rightarrow \Lambda^{\{M\}}$ is surjective. By the Grothendieck factorization theorem (cf. [64, 24.33]), there exist $C, \rho>0$ with

$$
\left\{\left(a_{k}\right):\left|\left(a_{k}\right)\right|_{1}^{M} \leq 1\right\} \subseteq j_{\{0\}}^{\infty}\left\{f:\|f\|_{[-1,1], \rho}^{M} \leq C\right\} .
$$

Thus there exist $f_{k} \in \mathcal{B}^{\{M\}}((-1,1))$ such that $f_{k}^{(j)}(0)=\delta_{j k}$ and

$$
\left\|f_{k}\right\|_{[-1,1], \rho}^{M} \leq \frac{C}{M_{k}}, \quad k \in \mathbb{N} .
$$

It is easy to modify the above proof to conclude again (4.1).

\subsection{Sufficiency.}

Theorem 4.4. Let $M$ be a strongly non-quasianalytic weight sequence. Then:

(1) $j_{\{0\}}^{\infty}: \mathcal{E}^{\{M\}}(\mathbb{R}) \rightarrow \Lambda^{\{M\}}$ is surjective.

(2) $j_{\{0\}}^{\infty}: \mathcal{E}^{(M)}(\mathbb{R}) \rightarrow \Lambda^{(M)}$ is split surjective, i.e., there exists a continuous linear right-inverse $E: \Lambda^{(M)} \rightarrow \mathcal{E}^{(M)}(\mathbb{R})$ with $j_{\{0\}}^{\infty} \circ E=\operatorname{id}_{\Lambda^{(M)}}$.

Remark 4.5. By [68] (see also [84, p.223]), the existence of a continuous linear right-inverse for $j_{\{0\}}^{\infty}: \mathcal{E}^{\{M\}}(\mathbb{R}) \rightarrow \Lambda^{\{M\}}$ is equivalent to (4.1) and additionally

$$
\forall \epsilon>0 \exists a \in \mathbb{N}_{>1}: \limsup _{k \rightarrow \infty}\left(\frac{M_{a k}}{M_{k}}\right)^{\frac{1}{k(a-1)}} \frac{1}{\mu_{a k}} \leq \epsilon .
$$

This condition is not easy to satisfy; for instance Gevrey sequences fail it. Indeed

$$
\left(\frac{G_{a k}^{s}}{G_{k}^{s}}\right)^{\frac{1}{k(a-1)}} \frac{1}{\gamma_{a k}^{s}} \geq\left((k(a-1)) !^{s}\right)^{\frac{1}{k(a-1)}} \frac{1}{(a k)^{s}} \geq\left(\frac{a-1}{e a}\right)^{s} .
$$

See also Section 9.4.

Let us concentrate on the more interesting Beurling case (2) in Theorem 4.4. We have to develop some tools before we can give the proof in Section 4.4.

4.3. The descendant of a non-quasianalytic weight sequence. A strongly non-quasianalytic weight sequence is equivalent to a strongly log-convex sequence, as will be shown in the next lemma. More generally, we may associate with any nonquasianalytic weight sequence $N$ a weight sequence $S$ with many good properties. The following construction was used in [78]; it is inspired by [68, Proposition 1.1].

Definition 4.6. Let $\nu=\left(\nu_{k}\right)$ be an increasing positive sequence with $\nu_{0}=1$ and $\sum_{k} \frac{1}{\nu_{k}}<\infty$. The descendant of $\nu$ is the positive sequence $\sigma=\sigma(\nu)$ defined by setting $\sigma_{0}:=1$,

$$
\tau_{k}:=\frac{k}{\nu_{k}}+\sum_{j \geq k} \frac{1}{\nu_{j}}, \quad k \geq 1
$$

and

$$
\sigma_{k}:=\frac{\tau_{1} k}{\tau_{k}}, \quad k \geq 1
$$

We shall also say that $S_{k}=\sigma_{0} \sigma_{1} \cdots \sigma_{k}$ is the descendant of $N_{k}=\nu_{0} \nu_{1} \cdots \nu_{k}$. 
Lemma 4.7. Let $\sigma$ be the descendant of $\nu$. Then:

(1) $\sup _{k} \frac{\sigma_{k}}{\nu_{k}}<\infty$.

(2) $\sup _{k} \frac{\sigma_{k}}{k} \sum_{j \geq k} \frac{1}{\nu_{j}}<\infty$.

(3) $1 \leq \frac{\sigma_{k}}{k}=: \sigma^{*} \nearrow \infty$.

(4) $\sigma$ is maximal among the sequences satisfying (1) and (2): If $\mu$ is an increasing positive sequence with $\sup _{k} \frac{\mu_{k}}{\nu_{k}}<\infty$ and $\sup _{k} \frac{\mu_{k}}{k} \sum_{j \geq k} \frac{1}{\nu_{j}}<\infty$, then $\sup _{k} \frac{\mu_{k}}{\sigma_{k}}<\infty$.

Proof. (1), (2), and (3) are immediate. The assumptions on $\mu$ in (4) imply $\tau_{k} \lesssim$ $\frac{k}{\nu_{k}}+\frac{k}{\mu_{k}} \lesssim \frac{k}{\mu_{k}}$ and hence $\mu_{k} \lesssim \sigma_{k}$.

Note that, by (3), the descendant $S$ of $N$ is a strongly log-convex weight sequence.

Corollary 4.8. The descendant $\sigma$ of $\nu$ satisfies

$$
\exists C>0 \forall k: C^{-1} \leq \frac{\sigma_{k}}{\nu_{k}} \leq C
$$

if and only if $N$ is strongly non-quasianalytic. In that case the weight sequences $S$ and $N$ are equivalent.

Proof. This follows from (1), (2), and (4) in Lemma 4.7.

Lemma 4.9. Let $N=\left(N_{k}\right)$ be a strongly non-quasianalytic strongly log-convex weight sequence and $S$ its descendant. Then, for all small $\epsilon>0, \tilde{S}:=\left(S_{k} / k !^{\epsilon}\right)$ is a strongly non-quasianalytic weight sequence.

Proof. Note that $\nu_{k}^{*}:=\frac{\nu_{k}}{k}$ is increasing. So, for $\sigma_{k}^{*}:=\frac{\sigma_{k}}{k}$ and $k \geq 1$,

$$
\frac{\sigma_{2 k}^{*}}{\sigma_{k}^{*}}=\frac{\tau_{k}}{\tau_{2 k}}=\frac{\frac{1}{\nu_{k}^{*}}+\sum_{j \geq k} \frac{1}{\nu_{j}}}{\frac{1}{\nu_{2 k}^{*}}+\sum_{j \geq 2 k} \frac{1}{\nu_{j}}} \geq \frac{\frac{1}{\nu_{2 k}^{*}}+\sum_{j \geq k} \frac{1}{\nu_{j}}}{\frac{1}{\nu_{2 k}^{*}}+\sum_{j \geq 2 k} \frac{1}{\nu_{j}}}=1+\frac{\sum_{k \leq j<2 k} \frac{1}{\nu_{j}}}{\frac{1}{\nu_{2 k}^{*}}+\sum_{j \geq 2 k} \frac{1}{\nu_{j}}} .
$$

Since $\sum_{k \leq j<2 k} \frac{1}{\nu_{j}} \geq \sum_{k \leq j<2 k} \frac{1}{\nu_{2 k}}=\frac{k}{\nu_{2 k}}$ and $\frac{1}{\nu_{2 k}^{*}}+\sum_{j \geq 2 k} \frac{1}{\nu_{j}} \lesssim \frac{1}{\nu_{2 k}^{*}}$, we find that

$$
q:=\inf _{k \geq 1} \frac{\sigma_{2 k}^{*}}{\sigma_{k}^{*}}>1
$$

Suppose that $\epsilon \in(0,1)$ is such that $q>2^{\epsilon}$. Then $\tilde{\sigma}_{k}^{*}:=\frac{\tilde{\sigma}_{k}}{k}=\frac{\sigma_{k}^{*}}{k^{\epsilon}}$ satisfies

$$
\tilde{q}:=\inf _{k \geq 1} \frac{\tilde{\sigma}_{2 k}^{*}}{\tilde{\sigma}_{k}^{*}}=\inf _{k \geq 1} \frac{\sigma_{2 k}^{*}}{\sigma_{k}^{*}} \cdot \frac{1}{2^{\epsilon}}>1 .
$$

The fact that $\sigma_{k}^{*}=\frac{\sigma_{k}}{k}$ is increasing implies that $\tilde{\sigma}_{k}$ is increasing. Thus,

$$
\sum_{j \geq k} \frac{1}{\tilde{\sigma}_{j}}=\sum_{\ell=0}^{\infty} \sum_{j=2^{\ell} k}^{2^{\ell+1} k-1} \frac{1}{\tilde{\sigma}_{j}} \leq \sum_{\ell=0}^{\infty} \frac{2^{\ell} k}{\tilde{\sigma}_{2^{\ell} k}}=\sum_{\ell=0}^{\infty} \frac{1}{\tilde{\sigma}_{2^{\ell} k}^{*}} \leq \frac{1}{\tilde{\sigma}_{k}^{*}} \sum_{\ell=0}^{\infty} \tilde{q}^{-\ell} .
$$

It follows that $\tilde{S}$ is a strongly non-quasianalytic weight sequence.

Corollary 4.10. Let $M=\left(M_{k}\right)$ be a strongly non-quasianalytic weight sequence. We may assume that $\tilde{\mu}:=\left(\mu_{k} / k^{\epsilon}\right)$ is increasing to infinity and satisfies (4.1) if $\epsilon>0$ is small enough. Hence the sequence

$$
\underbrace{\mu_{k}, \ldots, \mu_{k}}_{k \text { times }}, \mu_{k+1}\left(\frac{k}{k+1}\right)^{\epsilon}, \mu_{k+2}\left(\frac{k}{k+2}\right)^{\epsilon}, \ldots
$$


is increasing and for the sum of the reciprocals we have

$$
\frac{k}{\mu_{k}}+\sum_{j \geq k+1} \frac{1}{\mu_{j}}\left(\frac{j}{k}\right)^{\epsilon} \leq A \frac{k}{\mu_{k}}, \quad k \geq 1,
$$

for some constant $A>0$.

4.4. Proof of Theorem 4.4. We give a detailed proof of (2).

Claim. For each $k \in \mathbb{N}$, there exists $\psi_{k} \in \mathcal{D}^{(M)}(\mathbb{R})$ such that $\psi_{k}^{(j)}(0)=\delta_{j k}$ and

$$
\left\|\psi_{k}\right\|_{\mathbb{R}, \rho}^{M} \leq \frac{C_{\rho} H_{\rho}^{k}}{M_{k}} \quad \text { for all small } \rho>0
$$

Then $E: \Lambda^{(M)} \rightarrow \mathcal{E}^{(M)}(\mathbb{R})$ defined by $E: a=\left(a_{k}\right) \mapsto \sum_{k \geq 0} a_{k} \psi_{k}$ is the required right inverse of $j_{\{0\}}^{\infty}$. Indeed, for every $\sigma>0$ we have $|a|_{\sigma}^{M}<\infty$. Let $\rho>0$ (small) be given. Then

$$
\begin{aligned}
\left|\sum_{k \geq 0} a_{k} \psi_{k}^{(j)}\right| & \leq \sum_{k \geq 0}\left|a_{k}\right|\left|\psi_{k}^{(j)}\right| \\
& \leq \sum_{k \geq 0}|a|_{\sigma}^{M} \sigma^{k} M_{k} \cdot\left\|\psi_{k}\right\|_{\mathbb{R}, \rho}^{M} \rho^{j} M_{j} \\
& \leq \sum_{k \geq 0}|a|_{\sigma}^{M} \sigma^{k} M_{k} \cdot \frac{C_{\rho} H_{\rho}^{k}}{M_{k}} \rho^{j} M_{j}=|a|_{\sigma}^{M} \cdot C_{\rho} \rho^{j} M_{j} \cdot \sum_{k \geq 0}\left(\sigma H_{\rho}\right)^{k} .
\end{aligned}
$$

We may choose $\sigma=\sigma(\rho)>0$ such that $\sum_{k>0}\left(\sigma H_{\rho}\right)^{k}$ converges. It follows that $f:=E(a)=\sum_{k>0} a_{k} \psi_{k}$ defines a $C^{\infty}$-function $f$ on $\mathbb{R}$ such that for all $\rho>0$ there exist $\sigma>0$ and $\bar{C}>0$ such that

$$
\|f\|_{\mathbb{R}, \rho}^{M} \leq C|a|_{\sigma}^{M},
$$

in particular, $f \in \mathcal{E}^{(M)}(\mathbb{R})$. So the linear map $E$ is continuous and $j_{\{0\}}^{\infty} E(a)=a$ is clear from $\psi_{k}^{(j)}(0)=\delta_{j k}$.

Let us prove the claim. We apply Proposition 3.11 to the setup in Corollary 4.10 (for $K=\{0\}$ and $\sum d_{j}$ the left-hand side of (4.10)). So for each $k \geq 1$ there is a $C^{\infty}$-function $\varphi_{k}$ with $0 \leq \varphi_{k} \leq 1, \operatorname{supp} \varphi_{k} \subseteq\left(-A \frac{k}{\mu_{k}}, A \frac{k}{\mu_{k}}\right), \varphi_{k}(0)=1, \varphi_{k}^{(j)}(0)=0$ for all $j \geq 1$, and

$$
\left|\varphi_{k}^{(j)}\right| \leq \begin{cases}2^{j} \mu_{k}^{j} & \text { if } 0 \leq j \leq k, \\ 2^{j} \mu_{k}^{k} \frac{M_{j}}{M_{k}}\left(\frac{k^{j-k} k !}{j !}\right)^{\epsilon} & \text { if } j>k .\end{cases}
$$

Then $\psi_{k}(t):=\varphi_{k}(t) \frac{t^{k}}{k !}$ clearly satisfies $\psi_{k}^{(j)}(0)=\delta_{j k}$. It remains to show (4.11). If $0 \leq j \leq k$, then

$$
\begin{aligned}
\left|\psi_{k}^{(j)}\right| & \leq \sum_{i=0}^{j}\left(\begin{array}{c}
j \\
i
\end{array}\right) 2^{i} \mu_{k}^{i}\left(A \frac{k}{\mu_{k}}\right)^{k-j+i} \frac{1}{(k-j+i) !} \\
& \leq \frac{M_{j}}{M_{k}} \frac{\mu_{j+1} \cdots \mu_{k}}{\mu_{k}^{k-j}}(A e)^{k} \sum_{i=0}^{j}\left(\begin{array}{c}
j \\
i
\end{array}\right) 2^{i} A^{-j+i} \leq \frac{M_{j}}{M_{k}}(A e)^{k}\left(2+A^{-1}\right)^{j} .
\end{aligned}
$$


If $j \geq 2 k$, then

$$
\begin{aligned}
\left|\psi_{k}^{(j)}\right| & \leq \sum_{i=j-k}^{j}\left(\begin{array}{c}
j \\
i
\end{array}\right) 2^{i} \mu_{k}^{k} \frac{M_{i}}{M_{k}}\left(\frac{k^{i-k} k !}{i !}\right)^{\epsilon}\left(A \frac{k}{\mu_{k}}\right)^{k-j+i} \frac{1}{(k-j+i) !} \\
& \leq \frac{M_{j}}{M_{k}} \frac{\mu_{k}^{j-i} \mu_{k+1} \cdots \mu_{i}}{\mu_{k+1} \cdots \mu_{j}}(A e)^{k}\left(\frac{k^{j-2 k} k !}{(j-k) !}\right)^{\epsilon} \sum_{i=j-k}^{j}\left(\begin{array}{c}
j \\
i
\end{array}\right) 2^{i} A^{-j+i} \\
& \leq \frac{M_{j}}{M_{k}}(A e)^{k}\left(2+A^{-1}\right)^{j}\left(\frac{k^{j-2 k}}{(j-2 k) !}\right)^{\epsilon} .
\end{aligned}
$$

If $k<j<2 k$, then ( since $\left.\frac{k^{i-k} k !}{i !}=\frac{k^{i}}{i !} \frac{k !}{k^{k}} \leq e^{k}\right)$

$$
\begin{aligned}
\left|\psi_{k}^{(j)}\right| \leq & \sum_{i=0}^{k}\left(\begin{array}{c}
j \\
i
\end{array}\right) 2^{i} \mu_{k}^{i}\left(A \frac{k}{\mu_{k}}\right)^{k-j+i} \frac{1}{(k-j+i) !} \\
& +\sum_{i=k+1}^{j}\left(\begin{array}{c}
j \\
i
\end{array}\right) 2^{i} \mu_{k}^{k} \frac{M_{i}}{M_{k}}\left(\frac{k^{i-k} k !}{i !}\right)^{\epsilon}\left(A \frac{k}{\mu_{k}}\right)^{k-j+i} \frac{1}{(k-j+i) !} \\
\leq & \frac{M_{j}}{M_{k}}(A e)^{k}\left(2+A^{-1}\right)^{j}+\frac{M_{j}}{M_{k}}\left(A e^{2}\right)^{k}\left(2+A^{-1}\right)^{j} \\
\leq & \frac{M_{j}}{M_{k}} 2\left(A e^{2}\right)^{k}\left(2+A^{-1}\right)^{j} .
\end{aligned}
$$

Let $0<\rho<2+A^{-1}$ be fixed. For $j<2 k$, we get from (4.13) and (4.15) that

$$
\frac{\left|\psi_{k}^{(j)}\right|}{\rho^{j} M_{j}} \leq \frac{1}{M_{k}} 2\left(A e^{2}\right)^{k}\left(\frac{2+A^{-1}}{\rho}\right)^{j} \leq \frac{1}{M_{k}} 2\left(A e^{2}\right)^{k}\left(\frac{2+A^{-1}}{\rho}\right)^{2 k}
$$

and, for $j \geq 2 k,(4.14)$ gives

$$
\begin{aligned}
\frac{\left|\psi_{k}^{(j)}\right|}{\rho^{j} M_{j}} & \leq \frac{1}{M_{k}}(A e)^{k}\left(\frac{2+A^{-1}}{\rho}\right)^{j}\left(\frac{k^{j-2 k}}{(j-2 k) !}\right)^{\epsilon} \\
& =\frac{1}{M_{k}}(A e)^{k}\left(\frac{2+A^{-1}}{\rho}\right)^{2 k}\left(\frac{2+A^{-1}}{\rho}\right)^{j-2 k}\left(\frac{k^{j-2 k}}{(j-2 k) !}\right)^{\epsilon} \\
& =\frac{1}{M_{k}}(A e)^{k}\left(\frac{2+A^{-1}}{\rho}\right)^{2 k} \exp \left(k \epsilon\left(\frac{2+A^{-1}}{\rho}\right)^{1 / \epsilon}\right) .
\end{aligned}
$$

This completes the proof of the claim and hence of (2) in Theorem 4.4.

Let us briefly discuss the Roumieu case, that is (1) in Theorem 4.4. Given $a=\left(a_{k}\right) \in \Lambda^{\{M\}}$, which means that $|a|_{\rho}^{M}<\infty$ for some $\rho>0$, one looks for functions $\psi_{k}, k \in \mathbb{N}$, such that $f:=\sum_{k \geq 0} a_{k} \psi$ is of class $\mathcal{E}^{\{M\}}$ and $j_{\{0\}}^{\infty} f=a$. It suffices to repeat the above construction, where (4.9) is replaced by

$$
\underbrace{H \mu_{k}, \ldots, H \mu_{k}}_{k \text { times }}, H \mu_{k+1}, H \mu_{k+2}, \ldots
$$

If $H>0$ is chosen large enough, one finds that there is a constant $B \geq 1$ such that

$$
\|f\|_{\mathbb{R}, B H}^{M} \leq C(\rho)|a|_{\rho}^{M},
$$

but $H$ and thus also $\psi_{k}$ and $f$ depend on $\rho$; cf. Remark 4.5. 


\section{The Borel map in The quasianalytic Setting}

Let us investigate surjectivity of the Borel map in the quasianalytic case. Since there are no cutoff functions, we will restrict $j_{\{0\}}^{\infty}$ to the ring of germs of $\mathcal{E}^{[M]}$ functions at 0 which we denote by $\mathcal{E}_{0}^{[M]}$. (The dimension of the ambient space will be clear from the context.) A result of Carleman [22] states that the Borel map $j_{\{0\}}^{\infty}: \mathcal{E}_{0}^{[M]} \rightarrow \Lambda^{[M]}$ is never surjective if $M$ is a quasianalytic weight sequence with $m_{k}^{1 / k} \rightarrow \infty$. Recall that $m_{k}^{1 / k} \rightarrow \infty$ means that the real analytic class is strictly contained in $\mathcal{E}^{[M]}$; on the ring of real analytic germs the Borel map is an isomorphism $\mathcal{E}_{0}^{\{(k !)\}} \cong \Lambda^{\{(k !)\}}$.

Many different proofs of Carleman's result are known. We present a much stronger result which shows that certain elements of $\Lambda^{[M]}$ cannot be extended to germs of class $\mathcal{E}^{\{N\}}$, where $N$ is any quasianalytic weight sequence. It is based on a theorem of Bang [4] on the zeros of quasianalytic functions and their derivatives. See also [65] and [77].

\subsection{Zeros of quasianalytic functions and their derivatives.}

Theorem 5.1. Let $M$ be a quasianalytic weight sequence. Suppose that $f \in$ $C^{\infty}([0,1])$ is not identically zero and satisfies

$$
\left\|f^{(k)}\right\|_{[0,1]} \leq M_{k} \quad \text { for all } k \in \mathbb{N} .
$$

Suppose that for $0 \leq j \leq m$ there exists $x_{j} \in[0,1]$ such that $f^{(j)}\left(x_{j}\right)=0$. Then

$$
\sum_{j=1}^{m}\left|x_{j-1}-x_{j}\right| \geq \frac{1}{e} \sum_{\kappa<k \leq m} \frac{1}{\mu_{k}}
$$

where

$$
\kappa=-\log \sup _{j \geq 0} \frac{\left|f^{(j)}\left(x_{0}\right)\right|}{e^{j} M_{j}} .
$$

Proof. For $n \in \mathbb{N}$ and $t \in[0,1]$ consider $B_{f, n}(t):=\sup _{j \geq n} \frac{\left|f^{(j)}(t)\right|}{e^{j} M_{j}}$. Then:

(1) $B_{f, n}(t) \leq e^{-n}$ for all $t \in[0,1]$.

(2) $B_{f, n} \geq B_{f, n+1}$, and $f^{(n)}\left(t_{0}\right)=0$ implies $B_{f, n}\left(t_{0}\right)=B_{f, n+1}\left(t_{0}\right)$.

(3) For all $k>n$ and all $t, s \in[0,1]$,

$$
B_{f, n}(s)<\max \left\{B_{f, n}(t), e^{-k}\right\} e^{e|t-s| \mu_{k}} .
$$

(1) and (2) are obvious. To see (3) let $k>n, n \leq j<k$, and $t, s \in[0,1]$. Then, by Taylor's formula, for some $\xi$ between $t$ and $s$,

$$
\begin{aligned}
\frac{\left|f^{(j)}(s)\right|}{e^{j} M_{j}} & \leq \sum_{i=0}^{k-j-1} \frac{\left|f^{(j+i)}(t)\right||t-s|^{i}}{e^{j} M_{j} i !}+\frac{\left|f^{(k)}(\xi)\right||t-s|^{k-j}}{e^{j} M_{j}(k-j) !} \\
& =\sum_{i=0}^{k-j-1} \frac{M_{j+i}}{M_{j}} \frac{\left|f^{(j+i)}(t)\right|}{e^{j+i} M_{j+i}} \frac{(e|t-s|)^{i}}{i !}+e^{-k} \frac{M_{k}}{M_{j}} \frac{\left|f^{(k)}(\xi)\right|}{M_{k}} \frac{(e|t-s|)^{k-j}}{(k-j) !} \\
& \leq B_{f, N}(t) \sum_{i=0}^{k-j-1} \mu_{k}^{i} \frac{(e|t-s|)^{i}}{i !}+e^{-k} \mu_{k}^{k-j} \frac{(e|t-s|)^{k-j}}{(k-j) !} \\
& <\max \left\{B_{f, n}(t), e^{-k}\right\} e^{e|t-s| \mu_{k}}
\end{aligned}
$$


where we used that $\mu_{k}$ is increasing. If $j \geq k$, then trivially

$$
\frac{\left|f^{(j)}(s)\right|}{e^{j} M_{j}} \leq e^{-j}<\max \left\{B_{f, n}(t), e^{-k}\right\} e^{e|t-s| \mu_{k}} .
$$

This implies (3).

Let $f$ and $x_{j}$ be as in the theorem. Set $\tau_{k}:=\sum_{j=1}^{k}\left|x_{j-1}-x_{j}\right|, k \geq 1, \tau_{0}:=0$, and define for $t \in\left[\tau_{n-1}, \tau_{n}\right]$,

$$
\tilde{B}_{f, n}(t):= \begin{cases}B_{f, n}\left(x_{n-1}+\tau_{n-1}-t\right) & \text { if } x_{n}<x_{n-1}, \\ B_{f, n}\left(x_{n-1}-\tau_{n-1}+t\right) & \text { if } x_{n} \geq x_{n-1} .\end{cases}
$$

The function $\tilde{B}_{f, n}$ is continuous, by (3), and $\tilde{B}_{f, n}\left(\tau_{n}\right)=B_{f, n}\left(x_{n}\right)=B_{f, n+1}\left(x_{n}\right)=$ $\tilde{B}_{f, n+1}\left(\tau_{n}\right)$, by $(2)$. Thus,

$$
\tilde{B}_{f}(t):=\tilde{B}_{f, n}(t) \quad \text { if } t \in\left[\tau_{n-1}, \tau_{n}\right], n \geq 1 .
$$

defines a continuous function on $\left[0, \tau_{m}\right]$. By (1) and (2), we have $\tilde{B}_{f}(t) \leq e^{-n}$ for all $t \geq \tau_{n-1}$, in particular, $\tilde{B}_{f}(t) \leq e^{-m}$ for all $t \in\left[\tau_{m-1}, \tau_{m}\right]$. Since $f$ does not vanish identically, $\max _{t \in\left[0, \tau_{m}\right]} \tilde{B}_{f}(t) \geq \tilde{B}_{f}(0)=B_{f, 1}\left(x_{0}\right)=B_{f, 0}\left(x_{0}\right)>0$. Hence, the range of $\tilde{B}_{f}$ contains all numbers $e^{-k}$ for $\kappa<k \leq m$, where $e^{-\kappa}=B_{f, 0}\left(x_{0}\right)$ which is equivalent to (5.3). So we may choose a strictly increasing sequence $t_{k}, \kappa<k \leq m$, such that $\tilde{B}_{f}\left(t_{k}\right)=e^{-k}$ and $\tilde{B}_{f}(t)>e^{-k}$ for all $t \in\left(t_{k-1}, t_{k}\right)$ (recursively, take for $t_{k}$ the smallest $t \in \tilde{B}_{f}^{-1}\left(e^{-k}\right)$ with $t>t_{k-1}$ ). By (3) (applied to each interval in the subdivision of $\left(t_{k-1}, t_{k}\right)$ induced by the points $\tau_{n}$ between $t_{k-1}$ and $\left.t_{k}\right)$ we may conclude that

$$
\tilde{B}_{f}\left(t_{k-1}\right) \leq \tilde{B}_{f}\left(t_{k}\right) e^{e\left(t_{k}-t_{k-1}\right) \mu_{k}} .
$$

Since $\tilde{B}_{f}\left(t_{k}\right)=e^{-k}$, that means

$$
t_{k}-t_{k-1} \geq \frac{1}{e \mu_{k}}
$$

Summing over $k$ we find

$$
t_{m} \geq \frac{1}{e} \sum_{\kappa<k \leq m} \frac{1}{\mu_{k}}
$$

By the choice of the sequence $t_{k}$, we have $\tau_{k} \geq t_{k}$ which implies (5.2).

Corollary 5.2. Let $M$ be a quasianalytic weight sequence. Let $f \in C^{\infty}([0,1])$ satisfy (5.1). If $f^{(j)}(0)>0$ for all $j \in \mathbb{N}$, then $f^{(j)}(x)>0$ for all $x \in[0,1]$ and $j \in \mathbb{N}$.

Proof. Suppose that some derivative $f^{(j)}$ has a zero $x_{j} \in(0,1]$. By Rolle's theorem, we find a strictly decreasing sequence $x_{j}>x_{j+1}>\cdots>0$, where $f^{(k)}\left(x_{k}\right)=0$ for all $k \geq j$. This contradicts Theorem 5.1.

Corollary 5.3. Let $M$ be a quasianalytic weight sequence. Let $f \in C^{\infty}([0,1])$ satisfy (5.1). The total number of zeros (counted with multiplicities) of $f$ is bounded by

$$
\sup \left\{m \in \mathbb{N}: \sum_{\kappa<k \leq m} \frac{1}{\mu_{k}} \leq e\right\}+1 \text {. }
$$


Proof. Let $z_{1} \leq z_{2} \leq \cdots \leq z_{m}$ be the zeros of $f$ in $[0,1]$. By Rolle's theorem, there exists a sequence of points $z_{1}=x_{0} \leq x_{1} \leq \cdots \leq x_{m-1}$ in $[0,1]$ such that $f^{(j)}\left(x_{j}\right)=0$ for all $j$. By $(5.2)$,

$$
1 \geq \sum_{j=1}^{m-1}\left(x_{j}-x_{j-1}\right) \geq \frac{1}{e} \sum_{\kappa<k \leq m-1} \frac{1}{\mu_{k}}
$$

and the statement follows.

\subsection{The quasianalytic Borel map is never onto.}

Theorem 5.4. Let $M$ be a quasianalytic weight sequence such that $m_{k}^{1 / k} \rightarrow \infty$. Then there exist elements in $\Lambda^{(M)}$ that are not contained in $j_{\{0\}}^{\infty} \mathcal{B}^{\{N\}}((-r, r))$ for any quasianalytic weight sequence $N$ and any $r>0$.

Proof. Let $a=\left(a_{j}\right) \in \Lambda^{\{M\}}$ be positive, i.e., $a_{j}>0$ for all $j$. Let $N$ be any quasianalytic weight sequence and $r>0$. We claim that if there exists $f \in \mathcal{B}^{\{N\}}((-r, r))$ such that $j_{\{0\}}^{\infty} f=a$ then $f$ is real analytic. After rescaling we may assume that $\left\|f^{(j)}\right\|_{[0,1]} \leq N_{j}$ for all $j \in \mathbb{N}$. Corollary 5.2 implies that $f^{(j)}(x)>0$ for all $x \in[0,1]$ and all $j \in \mathbb{N}$. By Bernstein's theorem (e.g. [93, p. 146]), $f$ extends to an analytic function on the unit disk in $\mathbb{C}$. Now it suffices to choose $a=\left(a_{j}\right)$ such that it does not define a real analytic germ, which is possible by the assumption $m_{k}^{1 / k} \rightarrow \infty$.

To see that there exist such $a$ even in $\Lambda^{(M)}$ set $L_{k}:=k ! \sqrt{m_{k}}$ and let $\underline{L}$ be the log-convex minorant of $L$. Thus $\underline{L} \triangleleft M$ and $\underline{\ell}_{k}^{1 / k} \rightarrow \infty$ (cf. Footnote 2 ), so that $\underline{L}$ is a quasianalytic weight sequence for which the argument in the previous paragraph applies. $^{7}$

\subsection{The impossibility of quasianalytic extension.}

Theorem 5.5. Let $M$ be a quasianalytic weight sequence with $m_{k}^{1 / k} \rightarrow \infty$. Then $\mathcal{B}^{(M)}((-1,1))$ contains functions $f$ which have no quasianalytic extension to a larger interval, i.e., if $\tilde{f}$ is an extension of $f$ to a neighborhood of $[-1,1]$ and $N$ is any quasianalytic weight sequence, then $\tilde{f} \notin \mathcal{E}^{\{N\}}$.

Proof. We use an argument of $[65]$ to see that $\mathcal{B}^{\{M\}}((-1,1))$ contains functions with the asserted properties. That such functions can even be found in $\mathcal{B}^{(M)}((-1,1))$ follows from the reasoning at the end of the proof of Theorem 5.4. Let $c_{j}$ be a positive sequence with

$$
\begin{gathered}
c_{j}^{1 / j} \rightarrow 1, \\
\sum_{j=1}^{\infty} j^{n} c_{j} \leq M_{n} \quad \text { for all } n \in \mathbb{N} .
\end{gathered}
$$

The even function $f(x)=\sum_{k=0}^{\infty} c_{2 k} x^{2 k}$ is real analytic on $(-1,1)$, by $(5.4)$, and belongs to $\mathcal{B}^{\{M\}}((-1,1))$, by (5.5). Suppose that $f$ has a quasianalytic extension $\tilde{f}$ to a larger interval. Then $\tilde{f}^{(j)}(0)=f^{(j)}(0) \geq 0$ for all $j \in \mathbb{N}$. Hence $\tilde{f}$ is analytic on a disk centered at 0 with radius larger than 1, by Corollary 5.2 und Bernstein's theorem. But this contradicts (5.4).

\footnotetext{
${ }^{7}$ It is not hard to see that $\Lambda^{(M)}=\bigcup\left\{\Lambda^{\{L\}}: L\right.$ positive sequence, $\left.L \triangleleft M, \ell_{k}^{1 / k} \rightarrow \infty\right\}$.
} 
5.4. Digression: intricacies of the quasianalytic setting. We want to point out some interesting intricacies of the quasianalytic setting. This section deviates from the central theme of the article it is thus kept rather short.

(1) For each smooth germ $f$ there exist quasianalytic weight sequences $M^{1}, M^{2}$ such that $f=f_{1}+f_{2}$ with $f_{i} \in \mathcal{E}^{\left\{M^{i}\right\}}, i=1,2$. See [57] and [80] (who concluded from this that there is no largest o-minimal expansion of the real field).

(2) Weierstrass division and preparation, generally, fail in quasianalytic classes. See $[2,35,67]$. If $M$ is derivation-closed and quasianalytic, then division in $\mathcal{E}^{\{M\}}$ by a Weierstrass polynomial $\varphi$ holds if and only if all roots of $\varphi$ are real; see [30] and [29]. For an overview of related results see [88].

(3) Assume that $M$ is a quasianalytic strongly log-convex and derivation-closed weight sequence. It is not known if the local ring $\mathcal{E}_{0}^{[M]}$ is Noetherian. But $\mathcal{E}^{[M]}$ admits resolution of singularities [8,9] and also [80] and, consequently, tools such as topological Noetherianity, curve selection, and Łojasiewicz inequalities are available.

(4) Ultradifferentiable quasianalyticity cannot be tested in lower dimensions (in contrast to real analyticity $[86,11,12])$. Let $M$ be a quasianalytic strongly logconvex weight sequence with $m_{k}^{1 / k} \rightarrow \infty$. For any $n \geq 2$ and any positive sequence $N$ there exists a $C^{\infty}$-function $f \in \mathcal{E}^{[M]}\left(\mathbb{R}^{n} \backslash\{0\}\right) \backslash \mathcal{E}^{\{N\}}\left(\mathbb{R}^{n}\right)$ such that $f \circ p \in \mathcal{E}^{[M]}$ for all $\mathcal{E}^{[M]}$-mappings $p: \mathbb{R}^{m} \supseteq U \rightarrow \mathbb{R}^{n}$ with $m<n$. See [45] and [72].

(5) Quasianalytic Roumieu classes are intersections of non-quasianalytic ones: Let $M$ be a quasianalytic strongly log-convex weight sequence. Then

$$
\mathcal{E}^{\{M\}}=\bigcap_{N \in \mathcal{N}(M)} \mathcal{E}^{\{N\}},
$$

where $\mathcal{N}(M)$ is the collection of all non-quasianalytic weight sequences $N \geq M$. For suitable $M$ (e.g. Denjoy sequences $Q^{n, 1}$, see Example 3.10), we may even restrict the intersection to all strongly log-convex weight sequences in $\mathcal{N}(M)$. But notice that we cannot describe all $\mathcal{E}^{\{M\}}$ in this way: the intersection of all non-quasianalytic $\mathcal{E}^{\{N\}}$, where $N$ is strongly log-convex, is the quasianalytic class $\mathcal{E}^{\left\{Q^{1,1}\right\}} \supsetneq C^{\omega}$. See [13] and [49] as well as [66] for an application.

(6) Let $M$ be a quasianalytic, strongly log-convex, and derivation-closed weight sequence. Consider the quasianalytic equation

$$
\varphi(x, y)=y^{d}+a_{1}(x) y^{d-1}+\cdots a_{d}(x) \in \mathcal{E}_{0}^{[M]}[y], \quad x=\left(x_{1}, \ldots, x_{n}\right) .
$$

Due to [89], if $n=1$, then a smooth solution $y=h(x)$ is of class $\mathcal{E}^{[M]}$. It is not known, if this is true for $n>1$. There is the following partial solution [6]: Let $\varphi(x, y)$ be a function of class $\mathcal{E}^{\{M\}}$ defined near $(a, b) \in \mathbb{R}^{n} \times \mathbb{R}$. Then there exist $p \in \mathbb{N}$ and a quasianalytic class $\mathcal{Q} \subseteq \mathcal{E}^{\left\{M^{(p)}\right\}}$ (where $M_{k}^{(p)}:=M_{p k}$ ) such that if $\varphi(x, y)=0$ admits a formal power series solution $y=H(x)$ at $a$ then there is a solution $y=h(x) \in \mathcal{Q}$ near $a$ and $T_{a} h=H$. Note that, in general, $M^{(p)}$ is no longer quasianalytic and $\mathcal{Q}$ is not a Denjoy-Carleman class.

\section{BorEL'S LEMMA WITH CONTROLLED LOSS OF REGULARITY}

Let $M$ be a weight sequence satisfying $m_{k}^{1 / k} \rightarrow \infty$. We have seen that the Borel map $j_{\{0\}}^{\infty}: \mathcal{E}^{[M]}(\mathbb{R}) \rightarrow \Lambda^{[M]}$ is injective if and only if $M$ is quasianalytic 
and surjective if and only if $M$ is strongly non-quasianalytic. If $M$ is not strongly non-quasianalytic, then several new natural questions arise.

Question 6.1. Assume that $M$ is not strongly non-quasianalytic.

(1) What can be said about the image $j_{\{0\}}^{\infty} \mathcal{E}^{[M]}(\mathbb{R})$ ? Is there an intrinsic description?

(2) In the case that $M$ is quasianalytic, is there a constructive method for finding the unique function $f$ with $j_{\{0\}}^{\infty} f=a$ for a given $a \in j_{\{0\}}^{\infty} \mathcal{E}_{0}^{[M]}$ ?

(3) When do we have $\Lambda^{[L]} \subseteq j_{\{0\}}^{\infty} \mathcal{E}^{[M]}(\mathbb{R})$ for another weight sequence $L$ ?

Note that, as see in Theorem 5.4, positive sequences that grow fast enough cannot belong to $j_{\{0\}}^{\infty} \mathcal{E}_{0}^{[M]}$ if $M$ is quasianalytic.

6.1. When do we have $\Lambda^{[L]} \subseteq j_{\{0\}}^{\infty} \mathcal{E}^{[M]}(\mathbb{R})$ ? This question was completely answered by Schmets and Valdivia [85] if $M$ is a non-quasianalytic weight sequence.

Let $M \leq N$ be weight sequences, $N$ non-quasianalytic. For $p \in \mathbb{N}_{\geq 1}$ we consider the sequence $\lambda_{p}=\lambda_{p}(M, N)$ defined by

$$
\lambda_{p, k}:=\sup _{0 \leq j<k}\left(\frac{M_{k}}{p^{k} N_{j}}\right)^{\frac{1}{k-j}}, \quad k \geq 1 .
$$

Note that $\lambda_{p} \leq \mu$ for all $p \geq 1$, indeed, since $M \leq N$,

$$
\left(\frac{M_{k}}{p^{k} N_{j}}\right)^{\frac{1}{k-j}} \leq\left(\frac{M_{k}}{M_{j}}\right)^{\frac{1}{k-j}}=\left(\mu_{j+1} \cdots \mu_{k}\right)^{\frac{1}{k-j}} \leq \mu_{k}
$$

If $M$ has moderate growth, then $\mu_{k} \lesssim M_{k}^{1 / k}$ and so also a converse estimate holds:

$$
\mu_{k} \lesssim M_{k}^{1 / k}=p\left(\frac{M_{k}}{p^{k} N_{0}}\right)^{1 / k} \leq p \lambda_{p, k} .
$$

Theorem 6.2 ([85]). Let $M \leq N$ be weight sequences, $N$ non-quasianalytic. The following conditions are equivalent:

(1) $\Lambda^{(M)} \subseteq j_{\{0\}}^{\infty} \mathcal{E}^{(N)}(\mathbb{R})$.

(2) $\Lambda^{\{M\}} \subseteq j_{\{0\}}^{\infty} \mathcal{E}^{\{N\}}(\mathbb{R})$.

(3) There is $p \geq 1$ such that

$$
\sup _{k \geq 1} \frac{\lambda_{p, k}}{k} \sum_{j \geq k} \frac{1}{\nu_{j}}<\infty
$$

By the remarks before the theorem, if $M$ has moderate growth, then (6.1) (for any $p \geq 1$ ) is equivalent to

$$
\sup _{k \geq 1} \frac{\mu_{k}}{k} \sum_{j \geq k} \frac{1}{\nu_{j}}<\infty .
$$

In general, the latter condition is stronger than (6.1). By Lemma 4.7, the descendant $\sigma$ of $\nu$ is the largest sequence among all sequences $\mu$ satisfying $\mu \lesssim \nu$ and (6.2), in the sense that it generates the largest function space. 
6.2. Moment-type summation. Some interesting results concerning the questions 6.1(1) and (2) based on a moment-type summation method are due to Beurling, Carleson [25] and recently Kiro [46].

Informally, it works as follows. Let $I \subseteq \mathbb{R}$ be an open interval containing 0 . Given $f \in \mathcal{E}^{(M)}(I)$, the singular transform of $f$ is the entire function

$$
S_{M} f(z):=\sum_{n \geq 0} \frac{f^{(n)}(0)}{n ! m_{n+1}} z^{n}
$$

It depends only on $j_{\{0\}}^{\infty} f$ and hence studying the image $S_{M} \mathcal{E}^{(M)}(I)$ is equivalent to studying the image of the Borel map $j_{\{0\}}^{\infty} \mathcal{E}^{(M)}(I)$. This study is based on the observation that elements in $S_{M} \mathcal{E}^{(M)}(I)$ have a certain growth behavior in horizontal strips. (In the Roumieu case the series may have a finite radius of convergence and analytic continuation to a horizontal strip is required.)

Let $K$ be the kernel that solves the moment problem

$$
\int_{0}^{\infty} t^{n} K(t) d t=m_{n+1}, \quad n \in \mathbb{N} .
$$

Under suitable conditions, the regular transform

$$
R_{M} F(x):=\int_{0}^{\infty} F(x t) K(t) d t
$$

then yields the desired reconstruction $R_{M} S_{M} f=f$.

To make this approach work, one needs to control the asymptotic behavior of $K$ and of

$$
E(z):=\sum_{n \geq 0} \frac{z^{n}}{m_{n+1}}
$$

which manifests itself in regularity properties of the weights $M$. The function $E$ regulates the growth behavior of elements in $S_{M} \mathcal{E}^{(M)}(I)$ in horizontal strips.

Kiro [46] executes this program for a suitable class of weights; the case $M_{k}=$ $k ! \log (k+e)^{k}$ was treated by Beurling. Also a duality between suitable nonquasianalytic and quasianalytic classes, which have the same image under the respective singular transform, is explored.

\section{Optimal Cutoff FunCtions}

The most important tool for the extension problem for general closed subsets $A \subseteq \mathbb{R}^{n}$ are good cutoff functions. We start with an observation that shows how good ultradifferentiable cutoff functions can be.

7.1. A lower bound for cutoff functions. Cf. [20]. Let $M=\left(M_{k}\right)$ be a nonquasianalytic weight sequence. Let $r, \epsilon, \rho>0$. Suppose that $\varphi \in \mathcal{B}_{\rho}^{M}(\mathbb{R})$ satisfies

$$
\left.\varphi\right|_{[-r, r]}=1 \quad \text { and } \quad \operatorname{supp} \varphi \subseteq[-(1+\epsilon) r,(1+\epsilon) r] .
$$

It is to be expected that $\|\varphi\|_{\mathbb{R}, \rho}^{M}$ tends to infinity, if $r, \epsilon$, or $\rho$ approach 0 . Let us check and quantify this guess. For $t \in(r,(1+\epsilon) r)$, we have by Taylor's formula

$$
|\varphi(t)| \leq \frac{\varphi^{(k)}(\tau)}{k !}(r \epsilon)^{k}, \quad k \in \mathbb{N}
$$


for some $\tau \in(t,(1+\epsilon) r)$. Since $\varphi(t) \rightarrow 1$ as $t \rightarrow r$, we find $\left\|\varphi^{(k)}\right\|_{\mathbb{R}} \geq k ! /(r \epsilon)^{k}$ for all $k$. Thus,

$$
\|\varphi\|_{\mathbb{R}, \rho}^{M} \geq \sup _{k \in \mathbb{N}} \frac{k !}{(\rho r \epsilon)^{k} M_{k}}=\frac{1}{\inf _{k \in \mathbb{N}}(\rho r \epsilon)^{k} m_{k}} .
$$

It turns out that $h_{m}(t):=\inf _{k \in \mathbb{N}} m_{k} t^{k}$ is a very useful auxiliary function; we will discuss it in Section 7.2. So any cutoff function $\varphi \in \mathcal{B}_{\rho}^{M}(\mathbb{R})$ with (7.1) must satisfy

$$
\|\varphi\|_{\mathbb{R}, \rho}^{M} \geq \frac{1}{h_{m}(\rho r \epsilon)}
$$

7.2. Associated functions. Suppose that $m=\left(m_{k}\right)$ is log-convex, $1=m_{0} \leq m_{1}$ and $m_{k}^{1 / k} \rightarrow \infty$ (i.e., $m$ is a weight sequence or, equivalently, $M_{k}=k ! m_{k}$ is a strongly log-convex weight sequence). We associate the function $h_{m}:[0, \infty) \rightarrow$ $[0, \infty)$, where $h_{m}(0):=0$ and

$$
h_{m}(t):=\inf _{k \in \mathbb{N}} m_{k} t^{k}, \quad t>0 .
$$

Then $h_{m}$ is increasing, continuous, and positive for $t>0$. For $t \geq 1 / m_{1}$ we have $h_{m}(t)=1$. From $h_{m}$ we may recover the sequence $m$ by $m_{k}=\sup _{t>0} h_{m}(t) / t^{k}$. We also associate the counting function $\Gamma_{m}:(0, \infty) \rightarrow \mathbb{N}_{\geq 1}$ by setting

$$
\Gamma_{m}(t):=\min \left\{k \geq 1: h_{m}(t)=m_{k} t^{k}\right\}=\min \left\{k \geq 1: \frac{m_{k+1}}{m_{k}} \geq \frac{1}{t}\right\} ;
$$

for this identity we need that $\frac{m_{k+1}}{m_{k}}$ is increasing, i.e., $m$ is log-convex. Then:

$$
\begin{aligned}
& k \mapsto m_{k} t^{k} \text { is decreasing for } k \leq \Gamma_{m}(t), \\
& h_{m}(t)=m_{\Gamma_{m}(t)} t^{\Gamma_{m}(t)} \leq m_{k} t^{k} \text { for all } k .
\end{aligned}
$$

It follows that

$$
\Gamma_{m}(t)=k \quad \text { and } \quad h_{m}(t)=m_{k} t^{k}, \quad \text { for } t \in\left[\frac{m_{k}}{m_{k+1}}, \frac{m_{k-1}}{m_{k}}\right) .
$$

Example 7.1. For $m_{k}=k !^{s}$, where $s>0$, the function $h_{m}$ behaves like $t \mapsto$ $\exp \left(-1 / t^{1 / s}\right)$ :

$$
\sup _{k} \frac{t^{k}}{k !^{s}}=\left(\sup _{k} \frac{t^{k / s}}{k !}\right)^{s} \leq\left(\sum_{k} \frac{t^{k / s}}{k !}\right)^{s}=e^{s t^{1 / s}}
$$

and, conversely,

$$
e^{s t^{1 / s}}=\left(\sum_{k} \frac{1}{2^{k}} \frac{\left(2 t^{1 / s}\right)^{k}}{k !}\right)^{s} \leq 2^{s} \sup _{k} \frac{\left(2^{s} t\right)^{k}}{k !^{s}}
$$

Similarly, for $m_{k}=\log (k+e)^{s k}, h_{m}$ behaves like $t \mapsto \exp \left(-\exp \left(1 / t^{1 / s}\right)\right)$.

We shall need some properties of $h_{m}$ and $\Gamma_{m}$ which are guaranteed if $m$ has moderate growth:

Lemma 7.2. Suppose that $m$ is a weight sequence of moderate growth, i.e., there is a constant $C>1$ such that

$$
\frac{m_{2 k}}{m_{2 k-1}} \leq C \frac{m_{k}}{m_{k-1}}, \quad k \geq 1
$$


Then $^{8}$

$$
h_{m}(t) \leq h_{m}(C t)^{2}, \quad t>0,
$$

and, for a possibly other constant $C>1$,

$$
2 \Gamma_{m}(C t) \leq \Gamma_{m}(t), \quad t>0 \text { small enough. }
$$

Proof. Consider the function $\Sigma_{m}(t):=\left|\left\{k \geq 1: \frac{m_{k}}{m_{k-1}} \leq t\right\}\right|$. Now (7.8) implies

$$
2 \Sigma_{m}(t) \leq \Sigma_{m}(C t), \quad t>0
$$

It is well-known (cf. [58] and [47]) that $\omega_{m}$ defined by $\omega_{m}(t)=\log \left(1 / h_{m}(1 / t)\right)$ satisfies $^{9}$

$$
\omega_{m}(t)=\int_{0}^{t} \frac{\Sigma_{m}(u)}{u} d u .
$$

So (7.11) implies $2 \omega_{m}(t) \leq \omega_{m}(C t)$ for all $t>0$, which is clearly equivalent to (7.9).

Let us check (7.10). Since $m$ has moderate growth and is log-convex, there are constants $C_{1}, C_{2}>0$ such that

$$
\frac{m_{2 k+1}}{m_{2 k}} \leq C_{1} \frac{m_{2 k}}{m_{2 k-1}} \leq C_{2} \frac{m_{k}}{m_{k-1}} \leq C_{2} \frac{m_{k+1}}{m_{k}}, \quad k \geq 1 .
$$

Fix $t>0$ and let $\ell:=\Gamma_{m}(t)$. If $\ell=2 k$, then

$$
\frac{1}{t} \leq \frac{m_{\ell+1}}{m_{\ell}} \Longrightarrow \frac{1}{C_{2} t} \leq \frac{m_{k+1}}{m_{k}}
$$

that is, $\Gamma_{m}\left(C_{2} t\right) \leq k=\frac{1}{2} \Gamma_{m}(t)$. If $\ell=2 k+1$, then we similarly see that $\Gamma_{m}\left(\frac{C_{2}}{C_{1}} t\right) \leq$ $k=\frac{1}{2}\left(\Gamma_{m}(t)-1\right) \leq \frac{1}{2} \Gamma_{m}(t)$. Since $\Gamma_{m}$ is decreasing, (7.10) follows.

Moderate growth of the weight sequence is an essential technical tool in this section. So we make the following definition.

Definition 7.3. A weight sequence $M=\left(M_{k}\right)$ is called strongly regular if $M$ is strongly non-quasianalytic, has moderate growth, and $m$ is log-convex. Note that the last condition is actually for free: a strongly non-quasianalytic weight sequence $M$ is equivalent to a strongly log-convex weight sequence (that is still strongly non-quasianalytic), by Corollary 4.8.

7.3. Optimal cutoff functions of Roumieu type. It turns out that there exist optimal $\mathcal{E}^{\{M\}}$ cutoff functions, i.e., $\varphi \in \mathcal{E}^{\{M\}}(\mathbb{R})$ satisfying (7.1) and realizing (7.2) so that

$$
\|\varphi\|_{\mathbb{R}, \rho}^{M} \sim \frac{1}{h_{m}(\rho r \epsilon)}
$$

if and only if $M$ is strongly non-quasianalytic. The existence of $\varphi$ will follow from Proposition 3.11. The following lemma provides the necessary assumptions. Recall that $\mu_{k}^{*}=\frac{\mu_{k}}{k}=\frac{m_{k}}{m_{k-1}}$.

Lemma 7.4. Let $M$ be a strongly regular weight sequence. There is a constant $A \geq 1$ such that for each integer $p \geq 1$ there is a sequence $\left(\alpha_{k}^{p}\right)_{k \in \mathbb{N}}$ satisfying

$$
\sum_{k \geq 0} \frac{\alpha_{k}^{p}}{\alpha_{k+1}^{p}} \leq 1, \quad \alpha_{0}^{p}=1, \quad 0<\alpha_{k}^{p} \leq \frac{\left(\frac{A}{\mu_{p+1}^{*}}\right)^{k} M_{k}}{h_{m}\left(\frac{1}{e \mu_{p}^{*}}\right)} .
$$

\footnotetext{
${ }^{8}$ Actually (7.8) and (7.9) are equivalent, see [76, Remark 2.5].

${ }^{9}$ Note that the integral $\int_{1}^{\infty} \frac{\omega_{M}(t)}{t^{2}} d t$ converges if and only if $\int_{1}^{\infty} \frac{\Sigma_{M}(t)}{t^{2}} d t$ converges, and that is the case if and only if $M$ is non-quasianalytic; [47, Section 4].
} 
Proof. We will show that, for a suitable constant $A \geq 1$, the family of sequences

$$
\alpha_{k}^{p}:= \begin{cases}\left(\frac{A}{\mu_{p+1}^{*}}\right)^{k} M_{k} & \text { if } k>p, \\ (2 p)^{k} & \text { if } k \leq p,\end{cases}
$$

has the desired properties. Since $M$ has moderate growth, $\mu_{k+1} \lesssim M_{k}^{1 / k}$ and thus, for some constant $C \geq 1$,

$$
\alpha_{p}^{p}=2^{p} p^{p} \leq\left(\frac{2 C}{\mu_{p+1}^{*}}\right)^{p} M_{p} .
$$

So if $A$ is chosen large enough, then

$$
\sum_{k \geq 0} \frac{\alpha_{k}^{p}}{\alpha_{k+1}^{p}} \leq \sum_{k<p} \frac{1}{2 p}+\frac{\mu_{p+1}^{*}}{A} \sum_{k \geq p} \frac{1}{\mu_{k+1}} \leq 1
$$

since $M$ is strongly non-quasianalytic. The bound for $\alpha_{k}^{p}$ (in (7.13)) is obvious for $k>p$, since $h_{m} \leq 1$. If $k \leq p$, then, for large enough $A$,

$$
\frac{\alpha_{k}^{p}}{\left(\frac{A}{\mu_{p+1}^{*}}\right)^{k} M_{k}}=\frac{2^{k} p^{k}}{\left(\frac{A}{\mu_{p+1}^{*}}\right)^{k} M_{k}} \leq \frac{\mu_{p+1}^{k}}{\left(\frac{A}{2}\right)^{k} M_{k}} \leq \frac{\mu_{p}^{k}}{M_{k}} \leq \frac{\mu_{p}^{p}}{M_{p}}=\frac{\mu_{p}^{p}}{p ! m_{p}} \leq \frac{\left(e \mu_{p}^{*}\right)^{p}}{m_{p}} .
$$

This implies the statement.

Theorem 7.5 ([20, Theorem 2.2]). Let $M=\left(M_{k}\right)$ be a non-quasianalytic strongly log-convex weight sequence of moderate growth. Then the following conditions are equivalent:

(1) $M$ is strongly non-quasianalytic.

(2) There is a constant $C>0$ such that for all $\rho, r, \epsilon>0$ there exists $\varphi \in$ $\mathcal{E}^{\{M\}}(\mathbb{R})$ satisfying $(7.1)$ and

$$
\|\varphi\|_{\mathbb{R}, \rho}^{M} \leq \frac{1}{h_{m}(C \rho r \epsilon)} .
$$

Proof. (1) $\Rightarrow$ (2) We first consider the case $r=\epsilon=1$. Let $A$ be the constant from Lemma 7.4. Fix $0<\eta \leq 2 A$. Since $\mu_{k}^{*} \nearrow \infty$, there is an integer $p \geq 1$ such that

$$
\frac{2 A}{\mu_{p+1}^{*}}<\eta \leq \frac{2 A}{\mu_{p}^{*}}
$$

By Lemma 7.4, we may apply Proposition 3.11 ( to $d_{k+1}=\frac{\alpha_{k}^{p}}{\alpha_{k+1}^{p}}$ and $K=[-1,1]$ ) and get $\varphi=\varphi_{\eta} \in C^{\infty}(\mathbb{R})$ with $0 \leq \varphi \leq 1,\left.\varphi\right|_{[-1,1]}=1, \operatorname{supp} \varphi \subseteq[-2,2]$, and

$$
\left|\varphi^{(k)}(t)\right| \leq 2^{k} \alpha_{k}^{p} \leq \frac{\eta^{k} M_{k}}{h_{m}\left(\frac{\eta}{6 A}\right)}, \quad k \geq 1,
$$

by (7.13) and (7.15). For $\eta>2 A$ we put $\varphi_{\eta}:=\varphi_{2 A}$; then since $h_{m} \leq 1$,

$$
\left|\varphi^{(k)}(t)\right| \leq \frac{(2 A)^{k} M_{k}}{h_{m}\left(\frac{2 A}{6 A}\right)} \leq \frac{1}{h_{m}\left(\frac{1}{3}\right)} \frac{\eta^{k} M_{k}}{h_{m}\left(\frac{\eta}{6 A}\right)} .
$$

If $\delta:=1 / h_{m}\left(\frac{1}{3}\right)$ then for every $\eta>0$,

$$
\left|\varphi^{(k)}(t)\right| \leq \frac{(\delta \eta)^{k} M_{k}}{h_{m}\left(\frac{\eta}{6 A}\right)} .
$$


This implies (2), for $r=\epsilon=1$. For the general case it suffices to compose $\varphi_{\rho r \epsilon}$ with an odd smooth function $\theta: \mathbb{R} \rightarrow \mathbb{R}$ satisfying $\theta([-r, r])=[-1,1]$ and $\theta(x)=\frac{x+r \epsilon-r}{r \epsilon}$ for $x \geq r$.

$(2) \Rightarrow(1)$ Let $\varphi$ be as in (2) for given $r>0$ and $\rho=\epsilon=1$. Then the function $\psi(x):=\varphi(x-2 r) \cdot h_{m}(C r)$ satisfies $\left\|\psi^{(k)}\right\|_{\mathbb{R}} \leq M_{k}$ for all $k$ and vanishes on $(-\infty, 0]$. Lemma 3.2 implies

$$
h_{m}(C r)=|\psi(r)| \leq\left(\frac{2 r}{\sum_{k \geq \ell} \frac{1}{\mu_{k}}}\right)^{\ell}, \quad \ell \geq 1,
$$

provided that $r<\frac{1}{4} \sum_{k \geq \ell} \frac{1}{\mu_{k}}$. For those $\ell$ that satisfy $\frac{1}{C \mu_{\ell}^{*}}<\frac{1}{4} \sum_{k \geq \ell} \frac{1}{\mu_{k}}$, we obtain

$$
h_{m}\left(\frac{1}{\mu_{\ell}^{*}}\right) \leq\left(\frac{\frac{2}{C} \frac{1}{\mu_{\ell}^{*}}}{\sum_{k \geq \ell} \frac{1}{\mu_{k}}}\right)^{\ell} .
$$

On the other hand,

$$
h_{m}\left(\frac{1}{\mu_{\ell}^{*}}\right) \stackrel{(7.7)}{=} m_{\ell-1}\left(\frac{1}{\mu_{\ell}^{*}}\right)^{\ell-1} \geq H^{\ell-1}
$$

for some constant $H>0$, since $m$ has moderate growth. So we may conclude that $\sum_{k \geq \ell} \frac{1}{\mu_{k}} \lesssim \frac{1}{\mu_{\ell}^{*}}$ for all $\ell$.

7.4. Optimal cutoff functions of Beurling type. There exist also optimal cutoff functions of Beurling type. They will play a crucial role in Section 9.

Theorem 7.6. Let $M$ be a strongly regular weight sequence. Then there exist functions $\left(\varphi_{r}\right)_{r>0}$ in $\mathcal{E}^{(M)}(\mathbb{R})$ with the following properties:

(1) $0 \leq \varphi_{r} \leq 1$ for all $r>0$.

(2) $\left.\varphi_{r}\right|_{[-r, r]}=1$ and $\operatorname{supp} \varphi_{r} \subseteq\left[-\frac{9}{8} r, \frac{9}{8} r\right]$ for all $r>0$.

(3) For each $\rho>0$ there exist constants $A_{\rho}>0$ and $b_{\rho}>0$, such that

$$
\left\|\varphi_{r}\right\|_{\mathbb{R}, \rho}^{M} \leq \frac{A_{\rho}}{h_{m}\left(b_{\rho} r\right)}, \quad r>0 .
$$

The essential difference to Theorem 7.5 is that the cutoff functions may be taken independent of $\rho$. The dependence of the argument of $h_{m}$ on $\rho$ in (7.14) is however simpler than in (7.16); the linear dependence in (7.14) will be used in the proof of Theorem 8.4.

Proof. We may assume the setup of Corollary 4.10, in particular, there is a constant $A>0$ such that (4.10) holds. Let $0<r \leq 8 A$ be fixed and take an integer $k=k(r) \geq 1$ such that

$$
\frac{8 A}{\mu_{k}^{*}}<r \leq \frac{8 A}{\mu_{k-1}^{*}} .
$$

By Proposition 3.11 (applied to $K=[-r, r]$ and $\sum d_{j}$ the left-hand side of (4.10)), there exists $\varphi=\varphi_{r} \in C^{\infty}(\mathbb{R})$ with $0 \leq \varphi \leq 1,\left.\varphi\right|_{[-r, r]}=1, \operatorname{supp} \varphi \subseteq\left[-\frac{9}{8} r, \frac{9}{8} r\right]$, and

$$
\left|\varphi_{r}^{(j)}\right| \leq \begin{cases}2^{j} \mu_{k}^{j} & \text { if } 0 \leq j \leq k, \\ 2^{j} \mu_{k}^{k} \frac{M_{j}}{M_{k}}\left(\frac{k^{j-k} k !}{j !}\right)^{\epsilon} & \text { if } j>k .\end{cases}
$$

Since $m$ has moderate growth, we have $\mu_{k}^{*} \leq C \mu_{k-1}^{*}$ for some $C \geq 1$. Thus

$$
\frac{\mu_{k}^{k}}{M_{k}}=\frac{\mu_{k}^{k}}{k ! m_{k}} \leq \frac{\left(e \mu_{k}^{*}\right)^{k}}{m_{k}} \leq \frac{\left(C e \mu_{k-1}^{*}\right)^{k}}{m_{k}} \leq\left(\frac{8 A C e}{r}\right)^{k} \frac{1}{m_{k}}
$$


Let $\rho \in(0,1)$ be given. If $j \leq k$, then $\mu_{k}^{j} \leq \mu_{k}^{k} \frac{M_{j}}{M_{k}}$, and hence

$$
\left|\varphi_{r}^{(j)}\right| \leq 2^{j} M_{j}\left(\frac{8 A C e}{r}\right)^{k} \frac{1}{m_{k}} \leq \rho^{j} M_{j}\left(\frac{16 A C e}{r \rho}\right)^{k} \frac{1}{m_{k}} .
$$

If $j>k$, then $\frac{k^{j-k} k !}{j !} \leq \frac{k^{j-k}}{(j-k) !}$, and so

$$
\begin{aligned}
\left|\varphi_{r}^{(j)}\right| & \leq 2^{j} M_{j}\left(\frac{8 A C e}{r}\right)^{k} \frac{1}{m_{k}}\left(\frac{k^{j-k}}{(j-k) !}\right)^{\epsilon} \\
& =\rho^{j} M_{j}\left(\frac{16 A C e}{r \rho}\right)^{k} \frac{1}{m_{k}}\left(\frac{\left(k\left(\frac{2}{\rho}\right)^{1 / \epsilon}\right)^{j-k}}{(j-k) !}\right)^{\epsilon} \\
& \leq \rho^{j} M_{j}\left(\frac{16 A C e}{r \rho}\right)^{k} \frac{1}{m_{k}} e^{\epsilon k\left(\frac{2}{\rho}\right)^{1 / \epsilon}} \\
& =\rho^{j} M_{j} \frac{1}{\left(b_{\rho} r\right)^{k} m_{k}}, \quad \text { where } \quad b_{\rho}:=\frac{\rho}{16 A C e^{1+\epsilon\left(\frac{2}{\rho}\right)^{1 / \epsilon}}} .
\end{aligned}
$$

It follows that, for all $0<r \leq 8 \mathrm{~A}$ and all $\rho>0$,

$$
\left\|\varphi_{r}\right\|_{\mathbb{R}, \rho}^{M} \leq \frac{1}{h_{m}\left(b_{\rho} r\right)}
$$

for $\rho \geq 1$ the estimates are much simpler. For $r>8 A$ we apply Proposition 3.11 to $K=[-r, r]$ and (4.10) with $k=1$. This gives $\varphi_{r}$ having the same bounds as $\varphi_{8 A}$. Then

$$
\left\|\varphi_{r}\right\|_{\mathbb{R}, \rho}^{M} \leq \frac{1}{h_{m}\left(b_{\rho} 8 A\right)} \leq \frac{1}{h_{m}\left(b_{\rho} 8 A\right)} \frac{1}{h_{m}\left(b_{\rho} r\right)}=: \frac{A_{\rho}}{h_{m}\left(b_{\rho} r\right)}
$$

since $h_{m} \leq 1$.

7.5. Whitney cubes. We denote by $d_{A}(z)=d(z, A)=\inf \{|z-y|: y \in A\}$ the Euclidean distance of $z \in \mathbb{R}^{n}$ to some set $A \subseteq \mathbb{R}^{n}$.

Proposition 7.7 ([87, pp.167-170], [20]). For each closed non-empty set $A \subseteq \mathbb{R}^{n}$ there exists a countable collection of closed cubes $\left(Q_{j}\right)_{j \geq 1}$ with sides parallel to the coordinate axes and the following properties.

(1) $\mathbb{R}^{n} \backslash A=\bigcup_{j \geq 1} Q_{j}$.

(2) The interiors of the cubes $Q_{j}$ are pairwise disjoint.

(3) For each $j \geq 1$ we have

$$
\operatorname{diam} Q_{j} \leq \operatorname{dist}\left(Q_{j}, A\right) \leq 4 \operatorname{diam} Q_{j}
$$

(4) For each $j \geq 1$ let $Q_{j}^{*}$ be image of $Q_{j}$ under the dilation by the factor $\frac{9}{8}$ with center the center of $Q_{j}$. There exist constants $0<b_{0} \leq 1 \leq B_{0}$ such that for all $j \geq 1$ and all $z \in Q_{j}^{*}$,

$$
b_{0} \operatorname{diam} Q_{j} \leq d_{A}(z) \leq B_{0} \operatorname{diam} Q_{j} .
$$

(5) For each $j \geq 1$ the number of $i \geq 1$ with $Q_{i}^{*} \cap Q_{j}^{*} \neq \emptyset$ is bounded by $12^{2 n}$.

(6) There exist constants $0<b_{1} \leq 1 \leq B_{1}$ such that for all $i, j \geq 0$ with $Q_{i}^{*} \cap Q_{j}^{*} \neq \emptyset$ we have

$$
b_{1} \operatorname{diam} Q_{j} \leq \operatorname{diam} Q_{i} \leq B_{1} \operatorname{diam} Q_{j} .
$$

The constants $b_{0}, B_{0}, b_{1}, B_{1}$ are independent of $A$.

A collection $\left(Q_{j}\right)_{j \geq 1}$ with these properties is called a family of Whitney cubes for $A$. 
7.6. Optimal partitions of unity. Having optimal cutoff functions, it is a standard procedure to construct optimal partitions of unity subordinate to a given family of Whitney cubes. We begin with the Roumieu case.

Proposition 7.8. Let $M$ be a strongly regular weight sequence. Let $A \subseteq \mathbb{R}^{n}$ be a non-empty closed set and $\left(Q_{j}\right)_{j \geq 1}$ a family of Whitney cubes for $A$. Then there exists $C_{1} \geq 1$ such that for all $\epsilon>0$ there is a family of $C^{\infty}$-functions $\left(\varphi_{j, \epsilon}\right)_{j \geq 1}$ satisfying

(1) $0 \leq \varphi_{j, \epsilon} \leq 1$ for all $j \geq 1$,

(2) $\operatorname{supp} \varphi_{j, \epsilon} \subseteq Q_{j}^{*}$ for all $j \geq 1$,

(3) $\sum_{j \geq 1} \varphi_{j, \epsilon}(x)=1$ for all $x \in \mathbb{R}^{n} \backslash A$,

(4) for all $j \geq 1, \beta \in \mathbb{N}^{n}$, and $x \in \mathbb{R}^{n} \backslash A$,

$$
\left|\varphi_{j, \epsilon}^{(\beta)}(x)\right| \leq \frac{\epsilon^{|\beta|} M_{|\beta|}}{h_{m}\left(C_{1} \epsilon d_{A}(x)\right)} .
$$

Proof. By Theorem 7.5, there is a constant $C>1$ such that for each $\rho>0$ and each $r>0$ there exist functions $\chi_{\rho, r}$ such that

- $0 \leq \chi_{\rho, r}(x) \leq 1$ for all $x \in \mathbb{R}^{n}$,

- $\chi_{\rho, r}(x)=1$ for all $x \in[-r, r]^{n}$,

- $\chi_{\rho, r}(x)=0$ for all $x \notin\left[-\frac{9}{8} r, \frac{9}{8} r\right]^{n}$,

- for all $\beta \in \mathbb{N}^{n}$ and all $x \in \mathbb{R}^{n}$,

$$
\left|\chi_{\rho, r}^{(\beta)}(x)\right| \leq \frac{\rho^{|\beta|} M_{|\beta|}}{h_{m}(C \rho r)} .
$$

Let $x_{j}$ be the center and $2 s_{j}$ the sidelength of $Q_{j}$. Set

$$
\psi_{j, \epsilon}(x):=\chi_{\frac{\epsilon}{12^{2 n}}, s_{j}}\left(x-x_{j}\right) .
$$

Then $\psi_{j, \epsilon}$ is 1 on $Q_{j}$ and 0 outside $Q_{j}^{*}$. Moreover,

$$
\left|\psi_{j, \epsilon}^{(\beta)}(x)\right| \leq \frac{\left(\frac{\epsilon}{12^{2 n}}\right)^{|\beta|} M_{|\beta|}}{h_{m}\left(\frac{C \epsilon S_{j}}{12^{2 n}}\right)} \leq \frac{\left(\frac{\epsilon}{12^{2 n}}\right)^{|\beta|} M_{|\beta|}}{h_{m}\left(C_{0} \epsilon d_{A}(x)\right)}, \quad \text { for } C_{0}:=\frac{C}{2 \sqrt{n} 12^{2 n} B_{0}},
$$

since $d_{A}(x) \leq B_{0} \operatorname{diam} Q_{j}=2 \sqrt{n} B_{0} s_{j}$ if $x \in Q_{j}^{*}$, by Proposition 7.7(4). Now define $\left(\varphi_{j, \epsilon}\right)_{j \geq 1}$ by

$$
\varphi_{1, \epsilon}:=\psi_{1, \epsilon}, \quad \varphi_{j, \epsilon}:=\psi_{j, \epsilon} \prod_{k=1}^{j-1}\left(1-\psi_{k, \epsilon}\right), j \geq 2 .
$$

It is clear that $0 \leq \varphi_{j, \epsilon} \leq 1$ and $\operatorname{supp} \varphi_{j, \epsilon} \subseteq Q_{j}^{*}$ for all $j \geq 1$. To see (3) observe that, for all $j \geq 1$,

$$
\varphi_{1, \epsilon}+\cdots+\varphi_{j, \epsilon}=1-\left(1-\psi_{1, \epsilon}\right) \cdots\left(1-\psi_{j, \epsilon}\right) .
$$

If $x \in \mathbb{R}^{n} \backslash A$, then $x \in Q_{i_{x}}$ for some $i_{x} \geq 1$ and thus $\psi_{i_{x}, \epsilon}(x)=1$. Since the family $\left(\operatorname{supp} \varphi_{j, \epsilon}\right)_{j \geq 1}$ is locally finite, it follows that $\sum_{j \geq 1} \varphi_{j, \epsilon}(x)=1$ for all $x \in \mathbb{R}^{n} \backslash A$. Finally, let us check (4). In the product defining $\varphi_{j, \epsilon}$ at most $12^{2 n}$ factors are different from 1, by Proposition 7.7(5). Thus

$$
\left|\varphi_{j, \epsilon}^{(\beta)}(x)\right| \leq \frac{\epsilon^{|\beta|} M_{|\beta|}}{h_{m}\left(C_{0} \epsilon d_{A}(x)\right)^{12^{2 n}}} .
$$

By Lemma 7.2, there is a constant $D>1$ such that $h_{m}(t) \leq h_{m}(D t)^{12^{2 n}}$ for all $t>0$. Thus, we obtain (4) with $C_{1}=C_{0} / D$. 
Let us come to the Beurling case.

Proposition 7.9. Let $M$ be a strongly regular weight sequence. Let $A \subseteq \mathbb{R}^{n}$ be a non-empty closed set and $\left(Q_{j}\right)_{j \geq 1}$ a family of Whitney cubes for $A$. Then there is a family of $C^{\infty}$-functions $\left(\varphi_{j}\right)_{j \geq 1}$ satisfying

(1) $0 \leq \varphi_{j} \leq 1$ for all $j \geq 1$,

(2) $\operatorname{supp} \varphi_{j} \subseteq Q_{j}^{*}$ for all $j \geq 1$,

(3) $\sum_{j \geq 1} \varphi_{j}(x)=1$ for all $x \in \mathbb{R}^{n} \backslash A$,

(4) for each $\rho>0$ there exist $C_{1}, \tau_{1}>0$ such that for all $j \geq 1, \beta \in \mathbb{N}^{n}$, and $x \in \mathbb{R}^{n} \backslash A$,

$$
\left|\varphi_{j}^{(\beta)}(x)\right| \leq C_{1} \frac{\rho^{|\beta|} M_{|\beta|}}{h_{m}\left(\tau_{1} d_{A}(x)\right)} .
$$

Proof. By Theorem 7.6, there exist smooth functions $\left(\chi_{r}\right)_{r>0}$ such that

- $0 \leq \chi_{r}(x) \leq 1$ for all $x \in \mathbb{R}^{n}$,

- $\chi_{r}(x)=1$ for all $x \in[-r, r]^{n}$,

- $\chi_{r}(x)=0$ for all $x \notin\left[-\frac{9}{8} r, \frac{9}{8} r\right]^{n}$,

- for each $\rho>0$ there exist $A_{\rho} \geq 1$ and $b_{\rho}>0$ such that for all $\beta \in \mathbb{N}^{n}$ and $x \in \mathbb{R}^{n}$,

$$
\left|\chi_{r}^{(\beta)}(x)\right| \leq A_{\rho} \frac{\rho^{|\beta|} M_{|\beta|}}{h_{m}\left(b_{\rho} r\right)} .
$$

If $x_{j}$ denotes the center of the cube $Q_{j}$ and $2 s_{j}$ its sidelength, we set

$$
\psi_{j}(x):=\chi_{s_{j}}\left(x-x_{j}\right) .
$$

Then $\left.\psi_{j}\right|_{Q_{j}}=1$, supp $\psi_{j} \subseteq Q_{j}^{*}$, and

$$
\left|\psi_{j}^{(\beta)}(x)\right| \leq A_{\rho} \frac{\rho^{|\beta|} M_{|\beta|}}{h_{m}\left(b_{\rho} s_{j}\right)} \leq A_{\rho} \frac{\rho^{|\beta|} M_{|\beta|}}{h_{m}\left(b_{\rho}^{\prime} d_{A}(x)\right)} .
$$

In analogy to (7.17) we define the family $\left(\varphi_{j}\right)_{j \geq 1}$ which clearly has the properties (1)-(3). The fact that at most $12^{2 n}$ factors in the product defining $\varphi_{j}$ are different from 1 together with the estimates for $\psi_{j}$ and (7.9) easily imply (4).

\section{Extension of Whitney ultrajets}

If we restrict an $\mathcal{E}^{[M]}$-function defined on $\mathbb{R}^{n}$ to a closed subset of $\mathbb{R}^{n}$, then Taylor's theorem implies necessary conditions which lead to the notion of Whitney ultrajets.

8.1. Whitney ultrajets. Let $M=\left(M_{k}\right)$ be a weight sequence. Let $K \subseteq \mathbb{R}^{n}$ be a compact set. A Whitney ultrajet of class $\mathcal{E}^{\{M\}}$ on $K$ is a Whitney jet $F=\left(F^{\alpha}\right)_{\alpha} \in$ $\mathcal{E}(K)$ such that there exist $C>0$ and $\rho \geq 1$ with

$$
\begin{gathered}
\left|F^{\alpha}(x)\right| \leq C \rho^{|\alpha|} M_{|\alpha|}, \quad \alpha \in \mathbb{N}^{n}, x \in K, \\
\left|\left(R_{x}^{p} F\right)^{\alpha}(y)\right| \leq C \rho^{p+1}|\alpha| ! m_{p+1}|x-y|^{p+1-|\alpha|}, \quad p \in \mathbb{N},|\alpha| \leq p, x, y \in K .
\end{gathered}
$$

A Whitney ultrajet of class $\mathcal{E}^{(M)}$ on $K$ is a Whitney jet $F=\left(F^{\alpha}\right)_{\alpha} \in \mathcal{E}(K)$ with the property that for each $\rho>0$ there exists $C>0$ such that (8.1) and (8.2) hold.

The set of Whitney ultrajets of class $\mathcal{E}^{[M]}$ on $K$ is denoted by $\mathcal{E}^{[M]}(K)$. For a closed set $A \subseteq \mathbb{R}^{n}$ let

$$
\mathcal{E}^{[M]}(A):=\left\{F \in \mathcal{E}(A):\left.F\right|_{K} \in \mathcal{E}^{[M]}(K) \text { for all compact } K \subseteq A\right\}
$$


be the set of Whitney ultrajets of class $\mathcal{E}^{[M]}$ on $A$.

We endow $\mathcal{E}^{(M)}(A)$ with the projective limit topology with respect to the system of seminorms $p_{K, 1 / \ell}(F):=\|F\|_{K, 1 / \ell}^{M}+|F|_{K, 1 / \ell}^{M}, \ell \in \mathbb{N}_{\geq 1}$ and $K \subseteq A$ compact, where

$$
\|F\|_{K, \rho}^{M}:=\sup _{\alpha \in \mathbb{N}^{n}} \frac{\left\|F^{\alpha}\right\|_{K}}{\rho^{|\alpha|} M_{|\alpha|}}
$$

and $^{10}$

$$
|F|_{K, \rho}^{M}:=\sup _{\substack{x, y \in K \\ x \neq y}} \sup _{p \in \mathbb{N}} \sup _{|\alpha| \leq p}\left|\left(R_{x}^{p} F\right)^{\alpha}(y)\right| \frac{(p+1-|\alpha|) !}{|x-y|^{p+1-|\alpha|} \rho^{p+1} M_{p+1}} .
$$

Similarly, the Roumieu space

$$
\mathcal{E}^{\{M\}}(A)=\left\{F \in \mathcal{E}(A): \forall K \subseteq_{c p} A \exists m \in \mathbb{N}_{\geq 1}: p_{K, m}(F)<\infty\right\}
$$

is endowed with its natural locally convex topology. Sometimes we shall need the Banach space $\mathcal{E}_{\rho}^{M}(K):=\left\{F \in \mathcal{E}(K): p_{K, \rho}(F)<\infty\right\}$.

Remark 8.1. Let $k$ be a positive integer. A compact subset $K \subseteq \mathbb{R}^{n}$ is called Whitney $k$-regular if there is a constant $C>0$ such that for all $x, y \in K$ there exists a rectifiable path $\gamma$ in $K$ joining $x$ and $y$ such that

$$
\operatorname{length}(\gamma) \leq C|x-y|^{1 / k} \text {. }
$$

It is not difficult to see that (8.1) implies (8.2) provided that $K$ is Whitney 1regular, e.g., if $K$ is convex. This is no longer true if $K$ is only Whitney $k$-regular for some $k>1$; cf. [71, 1.9].

The goal of this section is to prove that Whitney ultrajets admit extensions preserving the class if the weight sequence $M$ is strongly regular. The proof of the Roumieu case is based on the existence of optimal cutoff functions and the extension problem for the singleton (the Borel map). The Beurling case can be extracted from the Roumieu case by a reduction argument based on Lemma 3.4.

In Section 9 we will prove that extension operators always exist in the Beurling case. As a by-product it gives an alternative direct proof that extensions exist.

8.2. Extension of Whitney ultrajets of Roumieu type. Let $A \subseteq \mathbb{R}^{n}$ be a non-empty closed set and $F \in \mathcal{E}(A)$ a Whitney jet on $A$. A function $f \in C^{\infty}\left(\mathbb{R}^{n}\right)$ is said to be a local extension of $F$ in $x$ if

$$
j_{\{x\}}^{\infty} f=F(x) .
$$

The next lemma provides local extensions in a uniform way.

Lemma 8.2 ([20], [15, Lemma 3.8]). Let $M$ be a strongly regular weight sequence. Let $K \subseteq \mathbb{R}^{n}$ be a compact non-empty set and $F \in \mathcal{E}^{\{M\}}(K)$. Then there exists $\rho>0$ such that for all $x \in K$ there exists $f_{x} \in \mathcal{B}^{\{M\}}\left(\mathbb{R}^{n}\right)$ with $j_{\{x\}}^{\infty} f_{x}=F(x)$ and

$$
\sup _{x \in K}\left\|f_{x}\right\|_{\mathbb{R}^{n}, \rho}^{M}<\infty .
$$

Proof. By assumption, $\{F(x): x \in K\}$ is a bounded subset of $\Lambda^{\{M\}}$. We know that $j_{\{0\}}^{\infty}: \mathcal{B}^{\{M\}}\left(\mathbb{R}^{n}\right) \rightarrow \Lambda^{\{M\}}$ is surjective, by Theorem 4.4. Since both spaces are Silva spaces, each bounded set in $\Lambda^{\{M\}}$ is the image of a bounded set in $\mathcal{B}^{\{M\}}\left(\mathbb{R}^{n}\right)$.

\footnotetext{
${ }^{10}$ Note that $|\alpha| ! m_{p+1} \leq \frac{M_{p+1}}{(p+1-|\alpha|) !} \leq 2^{p+1}|\alpha| ! m_{p+1}$.
} 
Thus, there is a bounded set $B$ in $\mathcal{B}^{\{M\}}\left(\mathbb{R}^{n}\right)$ such that for all $x \in K$ there exists $f_{x} \in B$ with $j_{\{x\}}^{\infty} f_{x}=F(x)$. This also implies (8.5).

Lemma 8.3. In the setting of the previous lemma, there exist $D, \sigma>0$ such that for all $x, y \in K, z \in \mathbb{R}^{n}$ and $\alpha \in \mathbb{N}^{n}$ we have

$$
\left|\left(f_{x}-f_{y}\right)^{(\alpha)}(z)\right| \leq D \sigma^{|\alpha|} M_{|\alpha|} h_{m}(\sigma(|z-x|+|z-y|)) .
$$

Proof. Let $x, y \in K$ be fixed. Take $p \in \mathbb{N}$ and $|\alpha| \leq p$. We have

$$
\begin{aligned}
T_{x}^{p} F(z)-T_{y}^{p} F(z) & =\sum_{\beta \leq p} \frac{(z-x)^{\beta}}{\beta !}\left(T_{x}^{p} F-T_{y}^{p} F\right)^{(\beta)}(x) \\
& =\sum_{\beta \leq p} \frac{(z-x)^{\beta}}{\beta !}\left(R_{y}^{p} F-R_{x}^{p} F\right)^{\beta}(x)=\sum_{\beta \leq p} \frac{(z-x)^{\beta}}{\beta !}\left(R_{y}^{p} F\right)^{\beta}(x) .
\end{aligned}
$$

Using (8.2), we find that there exist $A, \tau>0$ such that

$$
\begin{aligned}
\left|\left(T_{x}^{p} F-T_{y}^{p} F\right)^{(\alpha)}(z)\right| & \leq A \tau^{p+1} M_{p+1} \frac{(|z-x|+|x-y|)^{p+1-|\alpha|}}{(p+1-|\alpha|) !} \\
& \leq A(2 \tau)^{p+1} M_{p+1} \frac{(|z-x|+|z-y|)^{p+1-|\alpha|}}{(p+1-|\alpha|) !}
\end{aligned}
$$

By Taylor's formula and $j_{\{x\}}^{\infty} f_{x}=F(x)$,

$$
f_{x}(z)-T_{x}^{p} F(z)=\sum_{|\beta|=p+1} \frac{(z-x)^{\beta}}{\beta !} f_{x}^{(\beta)}(x+t(z-x)), \quad t \in[0,1],
$$

and thus

$$
\left|\left(f_{x}-T_{x}^{p} F\right)^{(\alpha)}(z)\right| \leq C \rho^{p+1} M_{p+1} \frac{|z-x|^{p+1-|\alpha|}}{(p+1-|\alpha|) !}, \quad|\alpha| \leq p .
$$

It follows that, for suitable constants $D, \sigma_{1}>0$,

$$
\left|\left(f_{x}-f_{y}\right)^{(\alpha)}(z)\right| \leq D \sigma_{1}^{p+1} M_{p+1} \frac{(|z-x|+|z-y|)^{p+1-|\alpha|}}{(p+1-|\alpha|) !},
$$

for all $x, y \in K, z \in \mathbb{R}^{n}, p \in \mathbb{N}$, and $|\alpha| \leq p$. Using that $M$ has moderate growth, we infer

$$
\left|\left(f_{x}-f_{y}\right)^{(\alpha)}(z)\right| \leq D \sigma^{|\alpha|} M_{|\alpha|} \cdot(\sigma(|z-x|+|z-y|))^{p+1-|\alpha|} m_{p+1-|\alpha|}
$$

for all $p \geq|\alpha|$. This implies (8.6).

Theorem 8.4. Let $M$ be a strongly regular weight sequence. For each non-empty compact set $K \subseteq \mathbb{R}^{n}$ the mapping $j_{K}^{\infty}: \mathcal{E}^{\{M\}}\left(\mathbb{R}^{n}\right) \rightarrow \mathcal{E}^{\{M\}}(K)$ is surjective.

Proof. Let $F \in \mathcal{E}^{\{M\}}(K)$ satisfy (8.1) and (8.2). By Lemma 8.2, there are $f_{x} \in \mathcal{B}^{\{M\}}\left(\mathbb{R}^{n}\right), x \in K$, satisfying $j_{\{x\}}^{\infty} f_{x}=F(x)$ for all $x \in K$ and (8.5). Let $\left(Q_{j}\right)_{j \geq 1}$ be a family of Whitney cubes for $K$. Let $\epsilon>0$ (to be specified later). By Proposition 7.8, there is a family of $C^{\infty}$-functions $\left(\varphi_{j, \epsilon}\right)_{j \geq 1}$ satisfying $0 \leq \varphi_{j, \epsilon} \leq 1$, $\operatorname{supp} \varphi_{j, \epsilon} \subseteq Q_{j}^{*}, \sum_{j} \varphi_{j, \epsilon}=1$ on $\mathbb{R}^{n} \backslash K$, and

$$
\left|\varphi_{j, \epsilon}^{(\beta)}(x)\right| \leq \frac{\epsilon^{|\beta|} M_{|\beta|}}{h_{m}\left(C_{1} \epsilon d_{K}(x)\right)}, \quad \beta \in \mathbb{N}^{n}, x \in \mathbb{R}^{n} \backslash K,
$$


where $C_{1}>0$ is independent of $\epsilon$. Let $x_{j}$ denote the center of $Q_{j}$ and let $\hat{x}_{j} \in K$ be such that $\left|x_{j}-\hat{x}_{j}\right|=d_{K}\left(x_{j}\right)$. We define

$$
f(z):= \begin{cases}\sum_{j \geq 1} \varphi_{j, \epsilon}(z) f_{\hat{x}_{j}}(z), & \text { if } z \in \mathbb{R}^{n} \backslash K, \\ F^{0}(z), & \text { if } z \in K .\end{cases}
$$

Clearly, $f$ is $C^{\infty}$ in $\mathbb{R}^{n} \backslash K$.

Claim. There exist constants $D_{1}, \sigma_{1}>0$ such that, for $x \in K$,

$$
\left|\left(f-f_{x}\right)^{(\alpha)}(z)\right| \leq D_{1} \sigma_{1}^{|\alpha|} M_{|\alpha|} h_{m}\left(\sigma_{1}|z-x|\right), \quad z \in \mathbb{R}^{n} \backslash K .
$$

We must estimate

$$
\left(f-f_{x}\right)^{(\alpha)}(z)=\sum_{\beta \leq \alpha}\left(\begin{array}{c}
\alpha \\
\beta
\end{array}\right) \sum_{j \geq 1} \varphi_{j, \epsilon}^{(\beta)}(z)\left(f_{\hat{x}_{j}}-f_{x}\right)^{(\alpha-\beta)}(z) .
$$

Let us first treat the term with $\beta=0$. If $z \in \operatorname{supp} \varphi_{j, \epsilon} \subseteq Q_{j}^{*}$, then

$$
\left|z-\hat{x}_{j}\right| \leq\left|z-x_{j}\right|+\left|x_{j}-\hat{x}_{j}\right| \leq \frac{9}{8} \operatorname{diam} Q_{j}+d_{K}\left(x_{j}\right) \lesssim d_{K}(z) \leq|z-x|,
$$

by Proposition 7.7(3)-(4). Thus, by Lemma 8.3, there exist $D, \sigma>0$ such that

$$
\sum_{j \geq 1} \varphi_{j, \epsilon}(z)\left|\left(f_{\hat{x}_{j}}-f_{x}\right)^{(\alpha)}(z)\right| \leq D \sigma^{|\alpha|} M_{|\alpha|} h_{m}(\sigma|z-x|) .
$$

Now we consider the terms with $\beta \neq 0$ in (8.11). To this end let $\hat{z}$ be a point in $K$ with $|\hat{z}-z|=d_{K}(z)$. Since $\beta \neq 0, \sum_{j \geq 1} \varphi_{j, \epsilon}^{(\beta)}(z)=0$ and thus

$$
\sum_{j \geq 1} \varphi_{j, \epsilon}^{(\beta)}(z)\left(f_{\hat{x}_{j}}-f_{x}\right)^{(\alpha-\beta)}(z)=\sum_{j \geq 1} \varphi_{j, \epsilon}^{(\beta)}(z)\left(f_{\hat{x}_{j}}-f_{\hat{z}}\right)^{(\alpha-\beta)}(z) .
$$

We infer from Lemma 8.3, (8.8), (8.12), and Proposition 7.7(5) that there exist constants $D, \sigma>0$ such that

$$
\begin{aligned}
\sum_{j \geq 1}\left|\varphi_{j, \epsilon}^{(\beta)}(z)\right|\left|\left(f_{\hat{x}_{j}}-f_{\hat{z}}\right)^{(\alpha-\beta)}(z)\right| & \leq 12^{2 n} D \epsilon^{|\beta|} \sigma^{|\alpha-\beta|} M_{|\beta|} M_{|\alpha-\beta|} \frac{h_{m}\left(\sigma d_{K}(z)\right)}{h_{m}\left(C_{1} \epsilon d_{K}(z)\right)} \\
& \stackrel{(7.9)}{\leq} 12^{2 n} D \epsilon^{|\beta|} \sigma^{|\alpha-\beta|} M_{|\alpha|} h_{m}\left(C \sigma d_{K}(z)\right)
\end{aligned}
$$

if we choose $\epsilon:=C \sigma / C_{1}$, where $C$ is the constant from (7.9). The claim follows.

In order to show that $f$ is a $C^{\infty}$-function on $\mathbb{R}^{n}$ with $j_{K}^{\infty} f=F$, let, for $\alpha \in \mathbb{N}^{n}$,

$$
f^{\alpha}(z):= \begin{cases}f^{(\alpha)}(z) & \text { if } z \in \mathbb{R}^{n} \backslash K, \\ F^{\alpha}(z) & \text { if } z \in K .\end{cases}
$$

We prove that $f=f^{0}$ is a $C^{\infty}$-function with $f^{(\alpha)}=f^{\alpha}$ for all $\alpha \in \mathbb{N}^{n}$. First we show that all $f^{\alpha}$ are continuous. This is clear near points $x \notin K$. So let $x \in K$. Now if $z \in K$, then

$$
\left|f^{\alpha}(z)-f^{\alpha}(x)\right|=\left|F^{\alpha}(z)-F^{\alpha}(x)\right|=\left|\left(R_{x}^{|\alpha|} F\right)^{\alpha}(z)\right| \rightarrow 0 \quad \text { as } z \rightarrow x,
$$

by (8.2). If $z \notin K$, then

$$
\begin{aligned}
\left|f^{\alpha}(z)-f^{\alpha}(x)\right| & =\left|f^{(\alpha)}(z)-f_{x}^{(\alpha)}(x)\right| \\
& \leq\left|f^{(\alpha)}(z)-f_{x}^{(\alpha)}(z)\right|+\left|f_{x}^{(\alpha)}(z)-f_{x}^{(\alpha)}(x)\right| \rightarrow 0 \quad \text { as } z \rightarrow x,
\end{aligned}
$$


by (8.10) and since $f_{x}^{(\alpha)}$ is continuous. At this point we may refer to the lemma of Hestenes [43, Lemma 1] or argue as follows: If $\delta_{i} \in \mathbb{N}^{n}$ denotes the multiindex with $\left(\delta_{i}\right)_{j}=\delta_{i j}$ (the Kronecker symbol), then for $x \in K$ and $z \in \mathbb{R}^{n}$,

$$
\left|f^{\alpha}(z)-f^{\alpha}(x)-\sum_{i=1}^{n}\left(z_{i}-x_{i}\right) f^{\alpha+\delta_{i}}(x)\right|=\left|f^{\alpha}(z)-\left(T_{x}^{|\alpha|+1} F\right)^{(\alpha)}(z)\right|=o(|z-x|),
$$

by (8.2), (8.7), and (8.10). It means that $f^{\alpha}$ is $C^{1}$ and $\partial_{x_{i}} f^{\alpha}=f^{\alpha+\delta_{i}}$. The assertion follows.

Finally, we claim that $f \in \mathcal{E}^{\{M\}}\left(\mathbb{R}^{n}\right)$. By (8.1), it suffices to consider $z \notin K$. Then the claim follows from

$$
\left|f^{(\alpha)}(z)\right| \leq\left|f_{\hat{z}}^{(\alpha)}(z)\right|+\left|\left(f-f_{\hat{z}}\right)^{(\alpha)}(z)\right|
$$

(8.5), and (8.10).

8.3. Extension of Whitney ultrajets of Beurling type. The Beurling case may be reduced to the Roumieu case by means of the next lemma.

Lemma 8.5 ([27, Proposition 17]). Let $M=\left(M_{k}\right)$ be a strongly regular weight sequence. For any non-negative sequence $L=\left(L_{k}\right)$ with $L \triangleleft M$ there exists a strongly regular weight sequence $N=\left(N_{k}\right)$ with $L \preccurlyeq N \triangleleft M$.

Proof. There is a decreasing sequence $\left(\epsilon_{k}\right)_{k \geq 1}$ tending to 0 with $L_{k} \leq \epsilon_{1} \cdots \epsilon_{k} M_{k}$ for all $k \geq 1$; it suffices to take $\epsilon_{k}:=\sup _{j \geq k}\left(\frac{L_{k}}{M_{k}}\right)^{1 / k}$. Since $M$ is strongly nonquasianalytic, there is a constant $C>0$ such that

$$
\sum_{j \geq k} \frac{1}{\mu_{k}} \leq C \frac{k}{\mu_{k}}, \quad k \in \mathbb{N}
$$

Applying Lemma 3.4 to $\alpha_{k}=\frac{1}{\mu_{k}}, \beta_{k}=\max \left\{\epsilon_{k}, \frac{k}{\mu_{k}}\right\}$, and $\gamma_{k}=\frac{k}{\mu_{k}}$, we find a sequence $\left(\theta_{k}\right)_{k \geq 1}$ with $\theta_{k} \nearrow \infty$ such that

$$
\sum_{j \geq k} \frac{\theta_{j}}{\mu_{j}} \leq 8 \theta_{k} \sum_{j \geq k} \frac{1}{\mu_{j}}, \quad \text { for all } k \geq 1,
$$

$\theta_{k} \epsilon_{k} \rightarrow 0$, and $\frac{k \theta_{k}}{\mu_{k}} \searrow 0$. Define $\nu_{k}:=\frac{\mu_{k}}{\theta_{k}}, k \geq 1$, and $\nu_{0}:=0$. Then $N_{k}:=\nu_{0} \nu_{1} \cdots \nu_{k}$ is a strongly log-convex weight sequence that is strongly nonquasianalytic, by (8.13) and (8.14), and has moderate growth,

$$
\frac{N_{j+k}}{N_{j} N_{k}}=\frac{M_{j+k}}{M_{j} M_{k}} \frac{\theta_{1} \cdots \theta_{j} \theta_{1} \cdots \theta_{k}}{\theta_{1} \cdots \theta_{j+k}} \leq \frac{M_{j+k}}{M_{j} M_{k}} .
$$

To see $L \preccurlyeq N \triangleleft M$ observe that $\left(\frac{L_{k}}{N_{k}}\right)^{1 / k}=\left(\frac{L_{k}}{M_{k}}\right)^{1 / k}\left(\theta_{1} \cdots \theta_{k}\right)^{1 / k} \leq\left(\theta_{1} \epsilon_{1} \cdots \theta_{k} \epsilon_{k}\right)^{1 / k}$ is bounded and $\left(\frac{M_{k}}{N_{k}}\right)^{1 / k}=\left(\theta_{1} \cdots \theta_{k}\right)^{1 / k} \rightarrow \infty$.

Theorem 8.6. Let $M$ be a strongly regular weight sequence. For each non-empty compact set $K \subseteq \mathbb{R}^{n}$ the mapping $j_{K}^{\infty}: \mathcal{E}^{(M)}\left(\mathbb{R}^{n}\right) \rightarrow \mathcal{E}^{(M)}(K)$ is surjective.

Proof. Let $F=\left(F^{\alpha}\right)_{\alpha} \in \mathcal{E}^{(M)}(K)$. Set $L_{0}:=\left\|F^{0}\right\|_{K}$ and

$$
L_{k}:=\max \left\{\sup _{|\alpha|=k}\left\|F^{\alpha}\right\|_{K}, \sup _{x \neq y \in K,|\alpha| \leq k-1} \frac{k !\left|\left(R_{x}^{k-1} F\right)^{\alpha}(y)\right|}{|\alpha| !|y-x|^{k-|\alpha|}}\right\}, \quad k \geq 1 .
$$

Then $L \triangleleft M$ and, by Lemma 8.5, there is a strongly regular weight sequence $N$ with $L \preccurlyeq N \triangleleft M$. So $F \in \mathcal{E}^{\{N\}}(K)$ and it has an extension $f \in \mathcal{E}^{\{N\}}\left(\mathbb{R}^{n}\right)$, by Theorem 8.4, which is also an element of $\mathcal{E}^{(M)}\left(\mathbb{R}^{n}\right)$. 
8.4. Extension from closed sets. It is now easy to get extension theorems from arbitrary closed subsets of $\mathbb{R}^{n}$.

Theorem 8.7. Let $M$ be a strongly regular weight sequence. For each non-empty closed set $A \subseteq \mathbb{R}^{n}$ the mapping $j_{A}^{\infty}: \mathcal{E}^{[M]}\left(\mathbb{R}^{n}\right) \rightarrow \mathcal{E}^{[M]}(A)$ is surjective.

Proof. This follows easily from Theorems 8.4 and 8.6, since $\mathcal{E}^{[M]}$ admits partitions of unity. Indeed, fix $F \in \mathcal{E}^{[M]}(A)$. For $k \in \mathbb{N}_{\geq 1}$ set $U_{k}:=\left\{x \in \mathbb{R}^{n}: k-2<|x|<k\right\}$; note that $U_{k}, k \geq 2$, are open shells and $U_{1}=\left\{x \in \mathbb{R}^{n}:|x|<1\right\}$. There exist functions $\varphi_{k} \in \mathcal{E}^{[M]}\left(\mathbb{R}^{n}\right)$ such that $0 \leq \varphi_{k} \leq 1$, supp $\varphi_{k} \subseteq U_{k}$, and $\sum_{k=1}^{\infty} \varphi_{k}=1$. For each $k \in \mathbb{N}_{\geq 1}$ the jet $F_{k}:=\left.F\right|_{\bar{U}_{k}}$ belongs to $\mathcal{E}^{[M]}\left(A \cap \bar{U}_{k}\right)$ and thus has an extension $f_{k} \in \mathcal{E}^{[M]}\left(\mathbb{R}^{n}\right)$, i.e., $j_{A \cap \bar{U}_{k}}^{\infty}\left(f_{k}\right)=F_{k}$. Then $f:=\sum_{k=1}^{\infty} \varphi_{k} f_{k}$ is a function in $\mathcal{E}^{[M]}\left(\mathbb{R}^{n}\right)$ since on any compact set the sum is finite. Each $x \in A$ belongs to at most two consecutive sets $U_{\ell}, U_{\ell+1}$. Thus, for each $\alpha \in \mathbb{N}^{n}$,

$$
\begin{aligned}
f^{(\alpha)}(x) & =\sum_{\beta \leq \alpha}\left(\begin{array}{l}
\alpha \\
\beta
\end{array}\right) \varphi_{\ell}^{(\beta)}(x) f_{\ell}^{(\alpha-\beta)}(x)+\sum_{\beta \leq \alpha}\left(\begin{array}{l}
\alpha \\
\beta
\end{array}\right) \varphi_{\ell+1}^{(\beta)}(x) f_{\ell+1}^{(\alpha-\beta)}(x) \\
& =\sum_{\beta \leq \alpha}\left(\begin{array}{l}
\alpha \\
\beta
\end{array}\right) \partial^{\beta}\left(\varphi_{\ell}(x)+\varphi_{\ell+1}(x)\right) F^{\alpha-\beta}(x)=F^{\alpha}(x),
\end{aligned}
$$

since all summands with $|\beta|>0$ vanish.

8.5. Analytic extensions. The ultradifferentiable extension in Theorem 8.7 can be made real analytic on the complement of $A$. This follows from a result of Schmets and Validivia [83]. A different proof due to Langenbruch [56, Theorem 13] is based on a general approximation theorem of Whitney type (cf. [92, Lemma 6]) which is interesting in its own right. In fact it is a special case of a quite general approximation theorem based on a surjectivity criterion for continuous linear maps between Fréchet spaces.

Theorem 8.8 ([56, Theorem 7]). Let $M=\left(M_{k}\right)$ be a non-quasianalytic weight sequence. Let $\Omega \subseteq \mathbb{R}^{n}$ be open and $\eta: \Omega \rightarrow(0, \infty)$ continuous. For any $f \in \mathcal{E}^{(M)}(\Omega)$ there exists $g \in \mathcal{H}\left(\Omega^{*}\right)$ such that

$$
\left|f^{(\alpha)}(x)-g^{(\alpha)}(x)\right| \leq \eta(x)^{|\alpha|+1} M_{|\alpha|} \quad \text { for all } x \in \Omega, \alpha \in \mathbb{N}^{n} .
$$

Here $\Omega^{*}:=\left\{z \in \mathbb{C}^{n}: \operatorname{Re}(z) \in \Omega,|\operatorname{Im}(z)|<\operatorname{dist}(\operatorname{Re}(z), \partial \Omega)\right\}$. We may assume that $\left.g\right|_{\Omega}$ is real-valued.

Theorem 8.9 ([56, Theorem 13]). Let $M=\left(M_{k}\right)$ be a non-quasianalytic weight sequence and $\Omega \subseteq \mathbb{R}^{n}$ open. There exists a continuous linear mapping $T: \mathcal{B}^{[M]}\left(\mathbb{R}^{n}\right) \rightarrow$ $\mathcal{B}^{[M]}\left(\mathbb{R}^{n}\right)$ such that $T(f)$ is an analytic $\Omega$-modification of $f$, i.e., $\left.T(f)\right|_{\Omega}$ is real analytic and $j_{A}^{\infty} T(f)=j_{A}^{\infty} f$, where $A:=\mathbb{R}^{n} \backslash \Omega$.

\section{Continuous linear extension operators}

In this section we address the question whether there exist continuous linear extension operators. We concentrate on the Beurling case for which the question has an affirmative answer. In Section 9.4 we comment briefly on the Roumieu case which has a negative answer most of the time. 
Let $M=\left(M_{k}\right)$ be a strongly regular weight sequence. Let $A \subseteq \mathbb{R}^{n}$ be a nonempty closed set. By Theorem 8.7, the following sequence is exact:

$$
0 \longrightarrow \operatorname{ker} j_{A}^{\infty} \longrightarrow \mathcal{E}^{(M)}\left(\mathbb{R}^{n}\right) \stackrel{j_{A}^{\infty}}{\longrightarrow} \mathcal{E}^{(M)}(A) \longrightarrow 0
$$

We will show that the sequence (9.1) splits: there exists a continuous linear rightinverse $E_{A}: \mathcal{E}^{(M)}(A) \rightarrow \mathcal{E}^{(M)}\left(\mathbb{R}^{n}\right)$ of $j_{A}^{\infty}$, that is $j_{A}^{\infty} \circ E_{A}=\operatorname{id}_{\mathcal{E}^{(M)}(A)}$. We say that $E_{A}$ is an extension operator.

We give an elementary constructive proof based on the fact that the Borel map is split surjective and on the existence of optimal cutoff functions. In Section 9.3 we shall briefly comment on the alternative approach based on the splitting theorem of Vogt and Wagner [91].

9.1. The existence of extension operators is a local property. We need a variant of Lemma 8.3.

Lemma 9.1. Let $M$ be a strongly regular weight sequence. For each $\rho>0$ there exist $C, \sigma>0$ such that the following holds. If $K \subseteq \mathbb{R}^{n}$ is compact and $H \subseteq \mathbb{R}^{n}$ is compact convex with $K \subseteq \operatorname{int} H, x_{1}, x_{2} \in K$, and $f_{1}, f_{2} \in C^{\infty}\left(\mathbb{R}^{n}\right)$ and $F \in \mathcal{E}(K)$ satisfy $j_{\left\{x_{1}\right\}}^{\infty} f_{1}=F\left(x_{1}\right), j_{\left\{x_{2}\right\}}^{\infty} f_{2}=F\left(x_{2}\right)$, and

$$
\left\|f_{1}\right\|_{H, \sigma}^{M}<\infty, \quad\left\|f_{2}\right\|_{H, \sigma}^{M}<\infty, \quad|F|_{K, \sigma}^{M}<\infty,
$$

then for all $z \in H \backslash K$ and $\alpha \in \mathbb{N}^{n}$,

$\left|\left(f_{1}-f_{2}\right)^{(\alpha)}(z)\right| \leq\left(\left\|f_{1}\right\|_{H, \sigma}^{M}+\left\|f_{2}\right\|_{H, \sigma}^{M}+|F|_{K, \sigma}^{M}\right) \cdot C \rho^{|\alpha|} M_{|\alpha|} h_{m}\left(\rho\left(\left|z-x_{1}\right|+\left|z-x_{2}\right|\right)\right)$.

Proof. As in the proof of Lemma 8.3 we find, for $|\alpha| \leq p$,

$$
\left|\left(T_{x_{1}}^{p} F-T_{x_{2}}^{p} F\right)^{(\alpha)}(z)\right| \leq|F|_{K, \sigma}^{M}(2 \sigma)^{p+1} M_{p+1} \frac{\left(\left|z-x_{1}\right|+\left|z-x_{2}\right|\right)^{p+1-|\alpha|}}{(p+1-|\alpha|) !} .
$$

Since $j_{\left\{x_{i}\right\}}^{\infty} f_{i}=F\left(x_{i}\right)$,

$$
\left|\left(f_{i}-T_{x_{i}}^{p} F\right)^{(\alpha)}(z)\right| \leq\left\|f_{i}\right\|_{H, \sigma}^{M} \sigma^{p+1} M_{p+1} \frac{\left(\left|z-x_{i}\right|\right)^{p+1-|\alpha|}}{(p+1-|\alpha|) !}, \quad i=1,2,
$$

as in (8.7). Since $M$ has moderate growth, $M_{p+1} \leq D^{p+1} M_{|\alpha|} M_{p+1-|\alpha|}$ for some $D>0$. Then the triangle inequality gives for all $p \geq|\alpha|$,

$$
\begin{gathered}
\left|\left(f_{1}-f_{2}\right)^{(\alpha)}(z)\right| \leq(2 \sigma D)^{|\alpha|} M_{|\alpha|} m_{p+1-|\alpha|}\left(2 \sigma D\left(\left|z-x_{1}\right|+\left|z-x_{2}\right|\right)\right)^{p+1-|\alpha|} \\
\times\left(\left\|f_{1}\right\|_{H, \sigma}^{M}+\left\|f_{2}\right\|_{H, \sigma}^{M}+|F|_{K, \sigma}^{M}\right)
\end{gathered}
$$

and the assertion follows easily.

Let $M$ be a strongly regular weight sequence. Let $A \subseteq \mathbb{R}^{n}$ be a closed non-empty set. A continuous linear mapping $E: \mathcal{E}^{(M)}(A) \rightarrow \mathcal{E}^{(M)}\left(\mathbb{R}^{n}\right)$ is said to be a local extension operator for $A$ in $x$ if

$$
j_{\{x\}}^{\infty} E(F)=F(x), \quad \text { for all } F \in \mathcal{E}^{(M)}(A) .
$$

Clearly, $E$ is an extension operator for $A$ if it is a local extension operator for $A$ in every $x \in A$. 
Proposition 9.2 ([37, Lemma 3.6]). Let $M$ be a strongly regular weight sequence. Let $A \subseteq \mathbb{R}^{n}$ be a closed non-empty set. Suppose that for each $x \in \partial A$ there exists a local extension operator $E_{x}$ for $A$ in $x$ and that $\left\{E_{x}: x \in \partial A\right\}$ is locally equicontinuous in $L\left(\mathcal{E}^{(M)}(A), \mathcal{E}^{(M)}\left(\mathbb{R}^{n}\right)\right)$. Then there exists an extension operator $E: \mathcal{E}^{(M)}(A) \rightarrow \mathcal{E}^{(M)}\left(\mathbb{R}^{n}\right)$.

Proof. Let $\left(Q_{j}\right)_{j \in \mathbb{N}}$ be a family of Whitney cubes for $A$ (cf. Proposition 7.7) and let $\left(\varphi_{j}\right)_{j \in \mathbb{N}}$ be the partition of unity provided by Proposition 7.9. For each $j$ choose a point $x_{j} \in \partial A$ such that $d_{Q_{j}}\left(x_{j}\right)=\operatorname{dist}\left(A, Q_{j}\right)$. For $F \in \mathcal{E}^{(M)}(A)$ define

$$
E(F)(z):= \begin{cases}\sum_{j \geq 1} \varphi_{j}(z) E_{x_{j}}(F)(z) & \text { if } z \in \mathbb{R}^{n} \backslash A, \\ F^{0}(z) & \text { if } z \in A .\end{cases}
$$

Clearly, $E(F)$ is of class $C^{\infty}$ on $\mathbb{R}^{n} \backslash A$. Recall that $p_{K, \sigma}(F):=\|F\|_{K, \sigma}^{M}+|F|_{K, \sigma}^{M}<\infty$ for all compact $K \subseteq A$ and $\sigma>0$.

Claim. For each compact convex $H \subseteq \mathbb{R}^{n}$ and for each $\rho>0$ there exist $C, \sigma, \tau>0$ and a compact subset $K \subseteq A$ such that for all $x \in \partial A \cap H, z \in H \backslash A$ with $|x-z| \leq \frac{b_{0}}{7}$ (with $b_{0}$ from Proposition 7.7), $F \in \mathcal{E}^{(M)}(A)$, and $\alpha \in \mathbb{N}^{n}$,

$$
\left|\left(E(F)-E_{x}(F)\right)^{(\alpha)}(z)\right| \leq C p_{K, \sigma}(F) \rho^{|\alpha|} M_{|\alpha|} h_{m}(\tau|x-z|) .
$$

Let us prove the claim. By Lemma 7.2, there is $C>1$ such that

$$
h_{m}(t) \leq h_{m}(C t)^{2}, \quad t>0 .
$$

Let a compact convex set $H \subseteq \mathbb{R}^{n}$ and $\rho>0$ be fixed. By Proposition 7.9, there exist positive $C_{1}=C_{1}(\rho)$ and $\tau_{1}=\tau_{1}(\rho)$ such that, for all $j$,

$$
\left|\varphi_{j}^{(\alpha)}(z)\right| \leq C_{1} \frac{\rho^{|\alpha|} M_{|\alpha|}}{h_{m}\left(\tau_{1} d_{A}(z)\right)}, \quad \alpha \in \mathbb{N}^{n}, z \in \mathbb{R}^{n} .
$$

Set

$$
\rho_{2}:=\min \left\{\rho, \frac{b_{0} \tau_{1}}{7 C}\right\}
$$

We invoke Lemma 9.1 for $\rho_{2}$ : there exist $C_{2}, \sigma_{2}>0$ such that the assertion of the lemma holds for $\rho_{2}, C_{2}, \sigma_{2}$ (instead of $\rho, C, \sigma$ in the lemma).

For $\epsilon>0$ let $H_{\epsilon}:=\left\{z \in \mathbb{R}^{n}: d_{H}(z) \leq \epsilon\right\}$ be the closed $\epsilon$-neighborhood of $H$. By assumption, the set $\left\{E_{x}: x \in \partial A \cap H_{2}\right\}$ is equicontinuous in $L\left(\mathcal{E}^{(M)}(A), \mathcal{E}^{(M)}\left(\mathbb{R}^{n}\right)\right)$. It follows that there exist constants $\sigma_{3} \geq \sigma_{2}, C_{3} \geq 1$ and a compact set $K \subseteq A$ with $A \cap H_{2} \subseteq K$ such that

$$
\left\|E_{x}(F)\right\|_{H_{2}, \sigma_{2}}^{M} \leq C_{3} p_{K, \sigma_{3}}(F), \quad F \in \mathcal{E}^{(M)}(A), x \in \partial A \cap H_{2} .
$$

Then, by the assertion of Lemma 9.1, for all $x, y \in \partial A \cap H_{1}, z \in H_{1} \backslash A$ and $\alpha \in \mathbb{N}^{n}$,

$$
\left|\left(E_{x}(F)-E_{y}(F)\right)^{(\alpha)}(z)\right| \leq C_{4} p_{K, \sigma_{3}}(F) \rho_{2}^{|\alpha|} M_{|\alpha|} h_{m}\left(\rho_{2}(|z-x|+|z-y|)\right),
$$

for some $C_{4} \geq 1$.

We are ready to estimate

$$
\left(E(F)-E_{x}(F)\right)^{(\alpha)}(z)=\sum_{\beta \leq \alpha}\left(\begin{array}{l}
\alpha \\
\beta
\end{array}\right) \sum_{j \geq 1} \varphi_{j}^{(\beta)}(z)\left(E_{x_{j}}(F)-E_{x}(F)\right)^{(\alpha-\beta)}(z),
$$

where $x \in \partial A \cap H$ and $z \in H \backslash A$ with $|x-z| \leq \frac{b_{0}}{7}$. By Proposition 7.7, for each $j \geq 1$ and $z \in Q_{j}^{*}$,

$$
\left|x_{j}-z\right| \leq d_{Q_{j}}\left(x_{j}\right)+\operatorname{diam} Q_{j}^{*}=\operatorname{dist}\left(A, Q_{j}\right)+\frac{9}{8} \operatorname{diam} Q_{j} \leq 6 \operatorname{diam} Q_{j} \leq \frac{6}{b_{0}} d_{A}(z) .
$$


Consequently (since $b_{0} \leq 1$ ),

$$
\left|x-x_{j}\right| \leq|x-z|+\left|z-x_{j}\right| \leq|x-z|+\frac{6}{b_{0}} d_{A}(z) \leq \frac{7}{b_{0}}|x-z| \leq 1
$$

and thus $x_{j} \in H_{1}$. So, by (9.6), for the term with $\beta=0$ we have

$$
\begin{aligned}
\sum_{j \geq 1} & \left|\varphi_{j}(z)\right|\left|\left(E_{x_{j}}(F)-E_{x}(F)\right)^{(\alpha)}(z)\right| \\
& \leq C_{4} p_{K, \sigma_{3}}(F) \rho_{2}^{|\alpha|} M_{|\alpha|} h_{m}\left(\rho_{2}\left(|z-x|+\left|z-x_{j}\right|\right)\right) \\
& \leq C_{4} p_{K, \sigma_{3}}(F) \rho_{2}^{|\alpha|} M_{|\alpha|} h_{m}\left(\frac{7 \rho_{2}}{b_{0}}|z-x|\right) \\
& \stackrel{(9.5)}{\leq} C_{4} p_{K, \sigma_{3}}(F) \rho^{|\alpha|} M_{|\alpha|} h_{m}\left(\tau_{1}|z-x|\right) .
\end{aligned}
$$

To estimate the terms with $\beta \neq 0$, let $y \in \partial A$ be such that $|y-z|=d_{A}(z)$. Then $\sum_{j \geq 1} \varphi_{j}^{(\beta)}(z)=0$ implies

$$
\sum_{j \geq 1} \varphi_{j}^{(\beta)}(z)\left(E_{x_{j}}(F)-E_{x}(F)\right)^{(\alpha-\beta)}(z)=\sum_{j \geq 1} \varphi_{j}^{(\beta)}(z)\left(E_{x_{j}}(F)-E_{y}(F)\right)^{(\alpha-\beta)}(z) .
$$

We have $|y-z|=d_{A}(z) \leq|z-x| \leq \frac{b_{0}}{7}$ and thus $y \in H_{1}$. Moreover, if $z \in Q_{j}^{*}$, then

$$
\left|z-x_{j}\right|+|z-y| \leq \frac{6}{b_{0}} d_{A}(z)+d_{A}(z) \leq \frac{7}{b_{0}} d_{A}(z) \leq \frac{7}{b_{0}}|x-z| \leq 1 .
$$

Thus, by (9.4) and (9.6),

$$
\begin{aligned}
\sum_{j \geq 1} \mid & \varphi_{j}^{(\beta)}(z)||\left(E_{x_{j}}(F)-E_{y}(F)\right)^{(\alpha-\beta)}(z) \mid \\
& \leq 12^{2 n} C_{1} C_{4} p_{K, \sigma_{3}}(F) \rho^{|\beta|} M_{|\beta|} \rho_{2}^{|\alpha-\beta|} M_{|\alpha-\beta|} \frac{h_{m}\left(\rho_{2}\left(\left|z-x_{j}\right|+|z-y|\right)\right)}{h_{m}\left(\tau_{1} d_{A}(z)\right)} \\
& \stackrel{(9.5)}{\leq} 12^{2 n} C_{1} C_{4} p_{K, \sigma_{3}}(F) \rho^{|\alpha|} M_{|\alpha|} \frac{h_{m}\left(\frac{\tau_{1}}{C} d_{A}(z)\right)}{h_{m}\left(\tau_{1} d_{A}(z)\right)} \\
& \stackrel{(9.3)}{\leq} 12^{2 n} C_{1} C_{4} p_{K, \sigma_{3}}(F) \rho^{|\alpha|} M_{|\alpha|} h_{m}\left(\tau_{1} d_{A}(z)\right) .
\end{aligned}
$$

Then the claim follows easily.

We are ready to complete the proof of the proposition. That $E(F)$ is a $C^{\infty}$ function on $\mathbb{R}^{n}$ with $j_{A}^{\infty} E(F)=F$ follows in analogy to the proof of Theorem 8.4.

It remains to show that $E(F) \in \mathcal{E}^{(M)}\left(\mathbb{R}^{n}\right)$ and that the linear map $E$ : $\mathcal{E}^{(M)}(A) \rightarrow \mathcal{E}^{(M)}\left(\mathbb{R}^{n}\right)$ is continuous. Let $H \subseteq \mathbb{R}^{n}$ be a compact convex set and $\rho>0$. Let $H_{b_{0} / 7}$ be the closed $\frac{b_{0}}{7}$-neighborhood of $H$. By the claim, there exist $C, \sigma, \tau>0$ and a compact set $K \subseteq A$ such that for all $x \in \partial A \cap H_{b_{0} / 7}, z \in H_{b_{0} / 7} \backslash A$ with $|x-z| \leq \frac{b_{0}}{7}, F \in \mathcal{E}^{(M)}(A)$ and $\alpha \in \mathbb{N}^{n}$ we have (9.2), in particular,

$$
\left|\left(E(F)-E_{x}(F)\right)^{(\alpha)}(z)\right| \leq C p_{K, \sigma}(F) \rho^{|\alpha|} M_{|\alpha|} .
$$

If $z \in H \backslash A$ satisfies $d_{A}(z) \leq \frac{b_{0}}{7}$, then there is $x \in \partial A \cap H_{b_{0} / 7}$ with $d_{A}(z)=|x-z|$. For such $z$ and $\alpha \in \mathbb{N}^{n}$,

$$
\begin{aligned}
\left|E(F)^{(\alpha)}(z)\right| & \leq\left|E_{x}(F)^{(\alpha)}(z)\right|+\left|E(F)^{(\alpha)}(z)-E_{x}(F)^{(\alpha)}(z)\right| \\
& \leq C p_{K, \sigma}(F) \rho^{|\alpha|} M_{|\alpha|},
\end{aligned}
$$

by (9.7) and by the assumption on local equicontinuity of $E_{x}$ (increasing $K, C$, and $\sigma$ if necessary). Together with (8.1) this shows the assertion. The proof is complete. 


\subsection{Extension operators always exist in the Beurling case.}

Theorem 9.3. Let $M$ be a strongly regular weight sequence. Let $A \subseteq \mathbb{R}^{n}$ be a nonempty closed set. There exists an extension operator $E: \mathcal{E}^{(M)}(A) \rightarrow \mathcal{E}^{(M)}\left(\mathbb{R}^{n}\right)$.

Proof. This follows from Theorem 4.4 and Proposition 9.2.

9.3. A different approach based on a splitting theorem for Fréchet spaces. Let $M=\left(M_{k}\right)$ be a strongly regular sequence. Let $K$ and $L$ be a non-empty compact subsets of $\mathbb{R}^{n}$ such that $K \subseteq \operatorname{int} L$. By Theorem 8.6, the sequence

$$
0 \longrightarrow \mathcal{D}^{(M)}(L, K) \longleftrightarrow \mathcal{D}^{(M)}(L) \stackrel{j_{K}^{\infty}}{\longrightarrow} \mathcal{E}^{(M)}(K) \longrightarrow 0
$$

is an exact sequence of Fréchet spaces, where we endow

$$
\mathcal{D}^{(M)}(L, K):=\left\{f \in \mathcal{E}^{(M)}\left(\mathbb{R}^{n}\right): \operatorname{supp} f \subseteq L, j_{K}^{\infty} f=0\right\}
$$

and $\mathcal{D}^{(M)}(L)$ with the subspace topology of $\mathcal{E}^{(M)}\left(\mathbb{R}^{n}\right)$. By the splitting theorem of Vogt and Wagner [91] (see also [64, 30.1]), the sequence splits if $\mathcal{E}^{(M)}(K)$ has property $(\mathrm{DN})$ and $\mathcal{D}^{(M)}(L, K)$ has property $(\Omega)$.

A Fréchet space $E$ with fundamental system of seminorms $\mathscr{P}=\left(p_{j}\right)_{j \in \mathbb{N}}$ has property $(\mathrm{DN})$ if

$$
\exists m \in \mathbb{N} \forall k \in \mathbb{N} \exists \ell \in \mathbb{N} \exists C>0: p_{k}^{2} \leq C p_{m} p_{\ell} .
$$

Then $p_{m}$ is a norm and every norm with this property is called a dominating norm. It has property $(\Omega)$ if

$$
\forall m \in \mathbb{N} \exists k \in \mathbb{N} \forall \ell \in \mathbb{N} \exists C>0 \exists \theta \in(0,1): p_{k}^{*} \leq C\left(p_{m}^{*}\right)^{1-\theta}\left(p_{\ell}^{*}\right)^{\theta},
$$

where $p_{k}^{*}(y):=\sup \left\{|y(x)|: p_{k}(x) \leq 1\right\}$ is the dual norm of $p_{k}$. The properties $(\mathrm{DN})$ and $(\Omega)$ are linear topological invariants. The property (DN) is inherited by all closed subspaces. ${ }^{11}$ A nuclear Fréchet space $E$ has property (DN) if and only if $E$ is isomorphic to a closed subspace of $s$ (the space of rapidly decreasing sequences). The property $(\Omega)$ is inherited by all quotient spaces. A nuclear Fréchet space $E$ has property $(\Omega)$ if and only if $E$ is isomorphic to a quotient space of $s$. Cf. $[64,29]$.

That $\mathcal{E}^{(M)}(K)$ has property $(\mathrm{DN})$ is a triviality. The fundamental system of seminorms

$$
p_{m}(F):=\|F\|_{K, 1 / m}^{M}+|F|_{K, 1 / m}^{M}, \quad m \in \mathbb{N}_{\geq 1},
$$

cf. (8.3) and (8.4), satisfies $p_{k}^{2} \leq p_{1} p_{k^{2}}$ for all $k \geq 1$, since

$$
\left(\frac{k^{|\alpha|}}{M_{|\alpha|}}\right)^{2}=\frac{k^{2|\alpha|}}{M_{|\alpha|}} \frac{1^{|\alpha|}}{M_{|\alpha|}} .
$$

That $\mathcal{D}^{(M)}(L, K)$ has property $(\Omega)$ follows from a result by Franken [37] (building on work of Meise and Taylor [63]). Both papers are situated in the setting of Braun-Meise-Taylor classes of which the Denjoy-Carleman classes form a subclass if $M=\left(M_{k}\right)$ is a strongly regular weight sequence; see Example 12.3(2).

\footnotetext{
${ }^{11}$ Hence, the splitting of $(9.8)$ implies that $\mathcal{E}^{(M)}(K)$ has the property (DN).
} 
9.4. Continuous linear extension operators in the Roumieu case. As already pointed out in Remark 4.5 in the case of the singleton, extension operators rarely exist in the Roumieu case. For instance, the condition (4.5) due to [68] shows that $j_{\{0\}}^{\infty}: \mathcal{E}^{\{M\}}\left(\mathbb{R}^{n}\right) \rightarrow \mathcal{E}^{\{M\}}(\{0\})=\Lambda^{\{M\}}$ has no continuous linear right-inverse if $M$ is any Gevrey sequence $M_{k}=G_{k}^{s}=k !^{s}, s>1$.

Actually, $j_{\{0\}}^{\infty}: \mathcal{E}^{\{M\}}\left([-1,1]^{n}\right) \rightarrow \Lambda^{\{M\}}$ is not split surjective for any strongly regular weight sequence $M$ as seen by the following topological argument (cf. [51, p.23]): $\mathcal{E}^{\{M\}}\left([-1,1]^{n}\right)$ is isomorphic to the dual of a power series space of finite type (cf. [52]), which cannot have $\Lambda^{\{M\}}$ as a complemented subspace, since $\Lambda^{\{M\}}$ is isomorphic to the dual of a power series space of infinite type (cf. [90, p.269]).

For the Whitney problem, Langenbruch [51] proved that for compact convex subsets $K, L$ of $\mathbb{R}^{n}$ with $\emptyset \neq \operatorname{int} K \subseteq K \subseteq \operatorname{int} L$ there is no extension operator $E: \mathcal{E}^{\{M\}}(K) \rightarrow \mathcal{E}^{\{M\}}(L)$ if $M$ is a weight sequence satisfying

$$
\begin{gathered}
\exists a>0 \forall b \in \mathbb{N}_{\geq a}: \bar{\lambda}(b):=\limsup _{k \rightarrow \infty} \frac{\mu_{b k}}{\mu_{k}} \leq a \liminf _{k \rightarrow \infty} \frac{\mu_{b k}}{\mu_{k}}=: a \underline{\lambda}(b), \\
\lim _{b \rightarrow \infty} \frac{\underline{\lambda}(b)}{b}=\infty .
\end{gathered}
$$

A weight sequence with these properties is strongly regular (cf. [51, pp.355-356] and [52, Lemma 1.1]). It is easy to check that the Gevrey sequences $G^{s}, s>1$, have the properties (9.9) and (9.10) (indeed, in that case $\bar{\lambda}(b)=\underline{\lambda}(b)=b^{s}$ ).

9.5. A different approach based on polynomial approximation. Denote by $\mathscr{P}_{d}$ the space of all polynomials on $\mathbb{R}^{n}$ of degree at most $d$, and let $\mathscr{P}_{-1}:=\{0\}$. For compact $K \subseteq \mathbb{R}^{n}$ and continuous $f: K \rightarrow \mathbb{R}$ we set

$$
\operatorname{dist}_{K}\left(f, \mathscr{P}_{d}\right):=\inf \left\{\|f-p\|_{K}: p \in \mathscr{P}_{d}\right\} .
$$

Let $M=\left(M_{k}\right)$ be a weight sequence. Let $\mathcal{A}^{\{M\}}(K)$ be the set of all $f \in C^{0}(K)$ such that there exists $\rho>0$ such that

$$
\|f\|_{K, \rho}^{\mathcal{A}^{M}}:=\sup _{k \in \mathbb{N}} \sup _{d \geq-1} \frac{(d+2)^{k} \operatorname{dist}_{K}\left(f, \mathscr{P}_{d}\right)}{\rho^{k} M_{k}}<\infty
$$

and $\mathcal{A}^{(M)}(K)$ the set of all $f \in C^{0}(K)$ such that (9.11) holds for all $\rho>0$. The spaces $\mathcal{A}^{[M]}(K)$ are endowed with their natural locally convex topologies.

The following theorem is an ultradifferentiable version of Jackson's theorem.

Theorem 9.4 ([71, Theorem 2.7]). Let $M$ be a derivation-closed weight sequence with $m_{k}^{1 / k} \rightarrow \infty$. For any compact $K \subseteq \mathbb{R}^{n}$ we have $j_{K}^{0} \mathcal{E}^{[M]}\left(\mathbb{R}^{n}\right) \subseteq \mathcal{A}^{[M]}(K)$, where $j_{K}^{0}(f)=\left.f\right|_{K}$. The inclusion is continuous.

Let $r \geq 1$. A compact set $K \subseteq \mathbb{R}^{n}$ is said to have the Markov property $\left(P_{r}\right)$ if there exist $C_{1}, C_{2}>0$ such that for every polynomial $p^{12}$

$$
|p(z)| \leq C_{1}\|p\|_{K} \quad \text { for } z \in \mathbb{C}^{n} \text { with } \operatorname{dist}(z, K) \leq \frac{C_{2}}{\operatorname{deg}(p)^{r}} .
$$

If $K \subseteq \mathbb{R}^{n}$ satisfies $\left(P_{r}\right)$, then necessarily $r \geq 2$, by [5], and $K$ is $C^{\infty}$-determining, see [70, Remark 3.5], that means $j_{K}^{0} f=0$ implies $j_{K}^{\infty} f=0$ for any $C^{\infty}$-function $f$.

\footnotetext{
${ }^{12}$ It is not hard to see that $\left(P_{r}\right)$ holds if and only if there is $C>0$ such that $\left\|p^{(\alpha)}\right\|_{K} \leq$ $C \operatorname{deg}(p)^{r|\alpha|}\|p\|_{K}$ for all $|\alpha| \geq 1$ and all polynomials $p$; cf. [70, Theorem 3.3]. Any compact subset of $\mathbb{R}^{n}$ that is uniformly polynomially cuspidal has the Markov property, in particular, any fat subanalytic compact subset of $\mathbb{R}^{n}$.
} 
The following extension theorem involves an unavoidable loss of regularity: for instance [39] shows that $j_{[-1,1]}^{0}: \mathcal{E}^{\left(G^{s}\right)}(\mathbb{R}) \rightarrow \mathcal{A}^{\left(G^{s}\right)}([-1,1])$ is not surjective. On the other hand the weight sequence is not required to be strongly non-quasianalytic.

Theorem 9.5 ([71]). Let $M$ be a non-quasianalytic weight sequence of moderate growth. Let $K \subseteq \mathbb{R}^{n}$ be a compact set satisfying $\left(P_{r}\right)$ with some $r \geq 2$. Then there exists a continuous linear operator $L: \mathcal{A}^{[M]}(K) \rightarrow \mathcal{E}^{\left[M^{r+1}\right]}\left(\mathbb{R}^{n}\right)$ such that $j_{K}^{0} \circ L=\mathrm{id}$. Here $M^{r+1}:=\left(M_{k}^{r+1}\right)$ is the $(r+1)$-st component-wise power of $M$.

Proof. By Proposition 3.11, there is a sequence $\left(\varphi_{k}\right)_{k \geq 1}$ of functions $\varphi_{k} \in C^{\infty}\left(\mathbb{R}^{n}\right)$ such that $0 \leq \varphi_{k} \leq 1, \operatorname{supp} \varphi_{k} \subseteq U_{k}:=\left\{x \in \mathbb{R}^{n}: d_{K}(x)<C_{2} k^{-r}\right\}$ (where $C_{2}$ is the constant from (9.12)), $\varphi_{k}=1$ in a neighborhood of $K$, and

$$
\left|\varphi_{k}^{(\alpha)}\right| \leq C(n)^{|\alpha|} k^{r|\alpha|} M_{|\alpha|}, \quad \alpha \in \mathbb{N}^{n}
$$

For $f \in \mathcal{A}^{[M]}(K)$ let $L_{d} f$ be the Lagrange interpolation polynomial of $f$ with nodes in Fekete-Leja extremal points of $K$ of order $d$; cf. [70, Section 2]. Then

$$
\left\|f-L_{d} f\right\|_{K} \leq 2(d+1)^{n} \operatorname{dist}_{K}\left(f, \mathscr{P}_{d}\right)
$$

Set

$$
L f(x):=\varphi_{1}(x) L_{1} f(x)+\sum_{d=1}^{\infty} \varphi_{d}(x)\left(L_{d+1} f(x)-L_{d} f(x)\right)
$$

and let us check that $L$ is well-defined and has the desired properties. By (9.12),

$$
\begin{aligned}
& \left|\partial^{\alpha}\left(\varphi_{d}(x)\left(L_{d+1} f(x)-L_{d} f(x)\right)\right)\right| \\
& \quad \leq \sum_{\beta \leq \alpha}\left(\begin{array}{c}
\alpha \\
\beta
\end{array}\right)\left|\varphi_{d}^{(\beta)}(x) \|\left(L_{d+1} f-L_{d} f\right)^{(\alpha-\beta)}(x)\right| \\
& \quad \leq C_{1} \sum_{\beta \leq \alpha}\left(\begin{array}{c}
\alpha \\
\beta
\end{array}\right)\left\|\varphi_{d}^{(\beta)}\right\|_{U_{d}}\left\|\left(L_{d+1} f-L_{d} f\right)^{(\alpha-\beta)}\right\|_{K} \\
& \quad \leq C C_{1} \sum_{\beta \leq \alpha}\left(\begin{array}{c}
\alpha \\
\beta
\end{array}\right) C(n)^{|\beta|} d^{r|\beta|} M_{|\beta|} \cdot(d+2)^{n+r|\alpha-\beta|} \operatorname{dist}_{K}\left(f, \mathscr{P}_{d}\right) \\
& \quad \leq D \sigma^{|\alpha|}(d+2)^{n+r|\alpha|} M_{|\alpha|} \operatorname{dist}_{K}\left(f, \mathscr{P}_{d}\right),
\end{aligned}
$$

for suitable constants $D, \sigma>0$. Thus, by (9.11) and moderate growth of $M$,

$$
\begin{aligned}
\left\|\partial^{\alpha}\left(\varphi_{d}\left(L_{d+1} f-L_{d} f\right)\right)\right\|_{\mathbb{R}^{n}} & \leq D \sigma^{|\alpha|}(d+2)^{n+r|\alpha|} M_{|\alpha|} \cdot\|f\|_{K, \rho}^{\mathcal{A}^{M}} \frac{\rho^{n+2+r|\alpha|} M_{n+2+r|\alpha|}}{(d+2)^{n+2+r|\alpha|}} \\
& \leq D_{1} \sigma_{1}^{|\alpha|} M_{|\alpha|}^{r+1}\|f\|_{K, \rho}^{\mathcal{A}^{M}} \cdot \frac{1}{(d+2)^{2}}
\end{aligned}
$$

It follows that $L f$ is a well-defined $C^{\infty}$-function satisfying

$$
\left\|(L f)^{(\alpha)}\right\|_{\mathbb{R}^{n}, \sigma_{1}}^{M^{r+1}} \leq D_{2}\|f\|_{K, \rho}^{\mathcal{A}^{M}},
$$

which implies the assertion; notice for the Beurling case that $\sigma_{1} \rightarrow 0$ as $\rho \rightarrow 0$. 


\section{Extension With CONTROLled LOSS OF REGUlARity}

If the weight sequence $M=\left(M_{k}\right)$ is not strongly non-quasianalytic, and thus extension preserving the class is not possible, it is natural to ask if the loss of regularity occurring in the extension can at least be controlled. This is indeed the case. It requires a new extension technique which is inspired by Dyn'kin's theory of almost analytic functions; cf. [33, 34] and also [40]. For the singleton see Theorem 6.2.

10.1. The setup: admissible pairs of weight sequences. Let $M, N$ be strongly log-convex weight sequences, $M$ of moderate growth with $m_{k}^{1 / k} \rightarrow \infty, N$ nonquasianalytic with $M \preccurlyeq N$, and

$$
\sup _{k} \frac{\mu_{k}}{k} \sum_{j \geq k} \frac{1}{\nu_{j}}<\infty,
$$

where $\mu_{k}=\frac{M_{k}}{M_{k-1}}$ and $\nu_{k}=\frac{N_{k}}{N_{k-1}}$. In that case we call $(M, N)$ an admissible pair. Note that $(M, M)$ is an admissible pair if and only if $M$ is strongly regular. Recall that the conditions (6.1) and (10.1) are equivalent if $M$ has moderate growth; so our definition is compatible with the case treated in Theorem 6.2.

Remark 10.1. Let $N$ be a strongly log-convex non-quasianalytic weight sequence of moderate growth and let $S$ be the descendant of $N$. Then $(S, N)$ is an admissible pair and, if $(M, N)$ is another admissible pair, then $M \preccurlyeq S$; see Lemma 4.7. Note that, if $M$ has moderate growth, then $M \preccurlyeq N$ if and only if $\mu \lesssim \nu$, indeed, $\mu_{k} \lesssim M_{k}^{1 / k} \lesssim N_{k}^{1 / k} \leq \nu_{k}$

10.2. Suitable cutoff functions. Let $(M, N)$ be an admissible pair of weight sequences. In analogy to the construction of optimal cutoff functions in Section 7.3 we obtain cutoff functions with bounds reflecting the weaker assumption (10.1).

Proposition 10.2 ([27, Proposition 4]). Let $(M, N)$ be an admissible pair of weight sequences. There is a constant $C>0$ such that for all $\rho, r, \epsilon>0$ there exists $\varphi \in \mathcal{E}^{\{N\}}(\mathbb{R})$ with $\left.\varphi\right|_{[-r, r]}=1, \operatorname{supp} \varphi \subseteq[-(1+\epsilon) r,(1+\epsilon) r]$, and

$$
\|\varphi\|_{\mathbb{R}, \rho}^{N} \leq \frac{1}{h_{m}(C \rho r \epsilon)} .
$$

Then it is straightforward to construct a partition of unity subordinate to a given family of Whitney cubes.

Proposition 10.3 ([27, Proposition 6]). Let $(M, N)$ be an admissible pair of weight sequences. Let $K \subseteq \mathbb{R}^{n}$ be a non-empty compact set and $\left(Q_{j}\right)_{j \geq 1}$ a family of Whitney cubes for $K$. Then there exists $C_{1} \geq 1$ such that for all $\epsilon>0$ there is a family of $C^{\infty}$-functions $\left(\varphi_{j, \epsilon}\right)_{j \geq 1}$ satisfying

(1) $0 \leq \varphi_{j, \epsilon} \leq 1$ for all $j \geq 1$,

(2) $\operatorname{supp} \varphi_{j, \epsilon} \subseteq Q_{j}^{*}$ for all $j \geq 1$,

(3) $\sum_{j \geq 1} \varphi_{j, \epsilon}(x)=1$ for all $x \in \mathbb{R}^{n} \backslash K$,

(4) for all $j \geq 1, \beta \in \mathbb{N}^{n}$, and $x \in \mathbb{R}^{n} \backslash K$,

$$
\left|\varphi_{j, \epsilon}^{(\beta)}(x)\right| \leq \frac{\epsilon^{|\beta|} N_{|\beta|}}{h_{m}\left(C_{1} \epsilon d_{K}(x)\right)} .
$$




\subsection{Extension of Whitney ultrajets.}

Theorem 10.4 ([27]). Let $M, N$ be strongly log-convex weight sequences, $M$ of moderate growth with $m_{k}^{1 / k} \rightarrow \infty$, and $N$ non-quasianalytic with $M \preccurlyeq N$. Then $(M, N)$ is admissible if and only if $\mathcal{E}^{\{M\}}(A) \subseteq j_{A}^{\infty} \mathcal{E}^{\{N\}}\left(\mathbb{R}^{n}\right)$ for all closed $A \subseteq \mathbb{R}^{n}$.

We will sketch the proof of the "only if" part. Assume that $(M, N)$ is admissible. The new feature is the shape of the extension formula: Let $K \subseteq \mathbb{R}^{n}$ be compact and $F=\left(F^{\alpha}\right)_{\alpha} \in \mathcal{E}^{\{M\}}(K)$ satisfy (8.1) and (8.2). Let $\left(Q_{j}\right)_{j \geq 1}$ be a family of Whitney cubes for $K$. Let $\epsilon, L>0$ be fixed. There is a family of $C^{\infty}$-functions $\left(\varphi_{j, \epsilon}\right)_{j \geq 1}$ satisfying the conclusion of Proposition 10.3. Let $x_{j}$ be the center of $Q_{j}$ and let $\hat{x}_{j} \in K$ be such that $\left|x_{j}-\hat{x}_{j}\right|=d_{K}\left(x_{j}\right)$. We define

$$
f(z):= \begin{cases}\sum_{j \geq 1} \varphi_{j, \epsilon}(z) T_{\hat{x}_{j}}^{2 \Gamma_{m}\left(L d_{K}\left(x_{j}\right)\right)} F(z), & \text { if } z \in \mathbb{R}^{n} \backslash K, \\ F^{0}(z), & \text { if } z \in K .\end{cases}
$$

(So the local extensions $f_{\hat{x}_{j}}$ in (8.9) are replaced by the Taylor polynomials $T_{\hat{x}_{j}}^{2 \Gamma_{m}\left(L d_{K}\left(x_{j}\right)\right)} F$.) The degree $p(x):=2 \Gamma_{m}\left(L d_{K}(x)\right)$ of the Taylor approximation tends to infinity as $x$ approaches the set $K$; the function $\Gamma_{m}$ was introduce in Section 7.2.

The proof that (10.2) is indeed the required extension is similar to the proof of Theorem 8.4. First we need estimates for the partial derivatives of the Taylor polynomials $T_{\hat{x}}^{p(x)} F$.

Lemma 10.5. There is a constant $A_{0}=A_{0}(M, N)>1$ such that for all $F \in$ $\mathcal{E}^{\{M\}}(K)$ satisfying (8.1) and (8.2) (with the constants $C, \rho$ ), all $L \geq A_{0} \rho, x \in \mathbb{R}^{n}$, and $\alpha \in \mathbb{N}^{n}$,

$$
\left|\left(T_{\hat{x}}^{p(x)} F\right)^{(\alpha)}(x)\right| \leq C(2 L)^{|\alpha|+1} M_{|\alpha|},
$$

and, if $|\alpha|<p(x)$,

$$
\left|\left(T_{\hat{x}}^{p(x)} F\right)^{(\alpha)}(x)-F^{\alpha}(\hat{x})\right| \leq C(2 L)^{|\alpha|+1}|\alpha| ! m_{|\alpha|+1} d_{K}(x) .
$$

Proof. For (10.3) we may restrict to the case $|\alpha| \leq p(x)$. By (8.1),

$$
\begin{aligned}
\left|\left(T_{\hat{x}}^{p(x)} F\right)^{(\alpha)}(x)\right| & \leq \sum_{\substack{\alpha \leq \beta \\
|\beta| \leq p(x)}} \frac{|x-\hat{x}|^{|\beta|-|\alpha|}}{(\beta-\alpha) !} C \rho^{|\beta|} M_{|\beta|} \\
& \leq C|\alpha| ! \sum_{\substack{\alpha \leq \beta \\
|\beta| \leq p(x)}} \frac{|\beta| !\left(n d_{K}(x)\right)^{|\beta|-|\alpha|}}{|\alpha| !(|\beta|-|\alpha|) !} \rho^{|\beta|} m_{|\beta|} \\
& \leq \frac{C|\alpha| !}{\left(n d_{K}(x)\right)^{|\alpha|}} \sum_{\substack{\alpha \leq \beta \\
|\beta| \leq p(x)}}\left(2 n \rho d_{K}(x)\right)^{|\beta|} m_{|\beta|} \\
& \leq \frac{C|\alpha| !}{\left(n d_{K}(x)\right)^{|\alpha|}} \sum_{j=|\alpha|}^{p(x)}\left(2 n^{2} \rho d_{K}(x)\right)^{j} m_{j},
\end{aligned}
$$

since the number of $\beta \in \mathbb{N}^{n}$ with $|\beta|=j$ is bounded by $n^{j}$. By Lemma 7.2,

there exists $\lambda<1$ such that $2 \Gamma_{m}(t) \leq \Gamma_{m}(\lambda t)$ for all $t>0$, 
and thus

$$
\left|\left(T_{\hat{x}}^{p(x)} F\right)^{(\alpha)}(x)\right| \leq \frac{C|\alpha| !}{\left(n d_{K}(x)\right)^{|\alpha|}} \sum_{j=|\alpha|}^{\Gamma_{m}\left(L \lambda d_{K}(x)\right)}\left(2 n^{2} \rho d_{K}(x)\right)^{j} m_{j} .
$$

Since $\left(L \lambda d_{K}(x)\right)^{j} m_{j} \leq\left(L \lambda d_{K}(x)\right)^{|\alpha|} m_{|\alpha|}$ for $|\alpha| \leq j \leq \Gamma_{m}\left(L \lambda d_{K}(x)\right)$, by (7.5),

$$
\left|\left(T_{\hat{x}}^{p(x)} F\right)^{(\alpha)}(x)\right| \leq C M_{|\alpha|}\left(\frac{L \lambda}{n}\right)^{|\alpha|} \sum_{j=|\alpha|}^{\Gamma_{m}\left(L \lambda d_{K}(x)\right)}\left(\frac{2 n^{2} \rho}{L \lambda}\right)^{j} .
$$

We obtain (10.3) if $L$ is chosen such that $\frac{2 n^{2} \rho}{L \lambda} \leq \frac{1}{2}$; then $A_{0}=\frac{4 n^{2}}{\lambda}$.

For (10.4) it suffices to note that, if $|\alpha|<p(x)$,

$$
\left(T_{\hat{x}}^{p(x)} F\right)^{(\alpha)}(x)-F^{\alpha}(\hat{x})=\sum_{\substack{\alpha \leq \beta \\|\alpha|<|\beta| \leq p(x)}} \frac{(x-\hat{x})^{\beta-\alpha}}{(\beta-\alpha) !} F^{\beta}(\hat{x}),
$$

and to follow the same arguments.

The core of the proof is the following lemma.

Lemma 10.6. There exist constants $A_{i}=A_{i}(M, N), i=1, \ldots, 4$, such that the following holds. If $\epsilon=A_{1} L$ and $L>A_{2} \rho$, then for all $x \in \mathbb{R}^{n} \backslash K$ with $d_{K}(x)<1$ and all $\alpha \in \mathbb{N}^{n}$ we have

$$
\left|\left(f-T_{\hat{x}}^{p(x)} F\right)^{(\alpha)}(x)\right| \leq C\left(L A_{3}\right)^{|\alpha|+1} N_{|\alpha|} h_{m}\left(L A_{4} d_{K}(x)\right) .
$$

Here $C, \rho$ are the constants from (8.1) and (8.2).

Proof. By the Leibniz rule we have

$$
\left(f-T_{\hat{x}}^{p(x)} F\right)^{(\alpha)}(x)=\sum_{\beta \leq \alpha}\left(\begin{array}{c}
\alpha \\
\beta
\end{array}\right) \sum_{j \geq 1} \varphi_{j, \epsilon}^{(\alpha-\beta)}(x)\left(T_{\hat{x}_{j}}^{p\left(x_{j}\right)} F-T_{\hat{x}}^{p(x)} F\right)^{(\beta)}(x) .
$$

Let us estimate

$$
\begin{aligned}
& \left|\left(T_{\hat{x}_{j}}^{p\left(x_{j}\right)} F-T_{\hat{x}}^{p(x)} F\right)^{(\beta)}(x)\right| \\
& \quad \leq\left|\left(T_{\hat{x}_{j}}^{p\left(x_{j}\right)} F-T_{\hat{x}}^{p\left(x_{j}\right)} F\right)^{(\beta)}(x)\right|+\left|\left(T_{\hat{x}}^{p\left(x_{j}\right)} F-T_{\hat{x}}^{p(x)} F\right)^{(\beta)}(x)\right|=: H_{1}+H_{2}
\end{aligned}
$$

for $x \in Q_{j}^{*}$ (since $\operatorname{supp} \varphi_{j, \epsilon} \subseteq Q_{j}^{*}$ ). One checks easily (as in the proof of Lemma 8.3) that, with $2 q:=p\left(x_{j}\right)$,

$$
H_{1} \leq C\left(2 n^{2} \rho\right)^{2 q+1}|\beta| ! m_{2 q+1}\left(\left|\hat{x}_{j}-x\right|+\left|\hat{x}_{j}-\hat{x}\right|\right)^{2 q+1-|\beta|} .
$$

By Proposition 7.7, there exist universal constants $0<b \leq 1 \leq B$ such that

$$
b d_{K}(x) \leq d_{K}\left(x_{j}\right) \leq B d_{K}(x), \quad \text { for } x \in Q_{j}^{*} .
$$

Moreover, $\left|\hat{x}_{j}-x\right|+\left|\hat{x}_{j}-\hat{x}\right| \lesssim d_{K}\left(x_{j}\right)$. So, using that $m$ has moderate growth,

$$
H_{1} \leq C\left(D_{1} \rho\right)^{2 q+1}|\beta| ! m_{q}^{2}\left(D_{2} d_{K}\left(x_{j}\right)\right)^{2 q+1-|\beta|},
$$

for constants $D_{i} \geq 1$ which depend only on $M, N$, and the dimension $n$. Since $h_{m}\left(L d_{K}\left(x_{j}\right)\right)=m_{q}\left(L d_{K}\left(x_{j}\right)\right)^{q} \leq m_{|\beta|}\left(L d_{K}\left(x_{j}\right)\right)^{|\beta|}$, by (7.6), we get

$$
H_{1} \leq C D_{1} D_{2} \rho\left(\frac{D_{1} D_{2} \rho}{L}\right)^{2 q} d_{K}\left(x_{j}\right)|\beta| ! m_{|\beta|} L^{|\beta|} h_{m}\left(L d_{K}\left(x_{j}\right)\right) .
$$


Since $d_{K}\left(x_{j}\right) \lesssim d_{K}(x)<1$ (by assumption), there is $D_{3}$ such that for $L>D_{3} \rho$,

$$
H_{1} \leq C L^{|\beta|+1} M_{|\beta|} h_{m}\left(L d_{K}\left(x_{j}\right)\right) \text {. }
$$

Now consider $H_{2}$. By (10.6) and (10.8), we have

$$
p\left(x_{j}\right)=2 \Gamma_{m}\left(L d_{K}\left(x_{j}\right)\right) \leq 2 \Gamma_{m}\left(L b d_{K}(x)\right) \leq \Gamma_{m}\left(L b \lambda d_{K}(x)\right)
$$

and similarly $p(x) \leq \Gamma_{m}\left(L b \lambda d_{K}(x)\right)$. So the polynomial $T_{\hat{x}}^{p\left(x_{j}\right)} F-T_{\hat{x}}^{p(x)} F$ has degree at most $\Gamma_{m}\left(L b \lambda d_{K}(x)\right)$. On the other hand, again by (10.8), the valuation of the polynomial $T_{\hat{x}}^{p\left(x_{j}\right)} F-T_{\hat{x}}^{p(x)} F$ is at least $2 \Gamma_{m}\left(L B d_{K}(x)\right)=: 2 r$. Thus, the calculation (10.5) gives

$$
H_{2} \leq \frac{C|\beta| !}{\left(n d_{K}(x)\right)^{|\beta|}} \sum_{j=2 r}^{\Gamma_{m}\left(L b \lambda d_{K}(x)\right)}\left(2 n^{2} \rho d_{K}(x)\right)^{j} m_{j} .
$$

For such $j$ we have $m_{j}\left(L b \lambda d_{K}(x)\right)^{j} \leq m_{2 r}\left(L b \lambda d_{K}(x)\right)^{2 r}$, by (7.5), and $h_{m}\left(L B d_{K}(x)\right)=m_{r}\left(L B d_{K}(x)\right)^{r} \leq m_{|\beta|}\left(L B d_{K}(x)\right)^{|\beta|}$, by (7.6). Using moderate growth of $m$, we may thus conclude that there is $D_{4} \geq 1$ such that, for $L>D_{4} \rho$,

$$
H_{2} \leq C L^{|\beta|+1} M_{|\beta|} h_{m}\left(L B d_{K}(x)\right) .
$$

In summary, for $x \in Q_{j}^{*}$ and $d_{K}(x)<1$,

$$
\left|\left(T_{\hat{x}_{j}}^{p\left(x_{j}\right)} F-T_{\hat{x}}^{p(x)} F\right)^{(\beta)}(x)\right| \leq C(2 L)^{|\beta|+1} M_{|\beta|} h_{m}\left(L B d_{K}(x)\right)
$$

and, consequently, in view of Proposition 10.3,

$$
\begin{aligned}
& \left|\left(f-T_{\hat{x}}^{p(x)} F\right)^{(\alpha)}(x)\right| \\
& \quad \leq \sum_{\beta \leq \alpha} \frac{\alpha !}{\beta !(\alpha-\beta) !} \cdot 12^{2 n} \cdot \frac{\epsilon^{|\alpha|-|\beta|} N_{|\alpha|-|\beta|}}{h_{m}\left(C_{1} \epsilon d_{K}(x)\right)} \cdot C(2 L)^{|\beta|+1} M_{|\beta|} h_{m}\left(L B d_{K}(x)\right) \\
& \quad \leq C 12^{2 n} \sum_{j=0}^{|\alpha|} \frac{|\alpha| ! n^{|\alpha|+j}}{j !(|\alpha|-j) !} \epsilon^{|\alpha|-j}(2 L)^{j+1} N_{|\alpha|-j} M_{j} \frac{h_{m}\left(L B d_{K}(x)\right)}{h_{m}\left(C_{1} \epsilon d_{K}(x)\right)} .
\end{aligned}
$$

Now, by (7.9),

$$
\frac{h_{m}\left(L B d_{K}(x)\right)}{h_{m}\left(C_{1} \epsilon d_{K}(x)\right)} \leq h_{m}\left(L B C d_{K}(x)\right)
$$

if we choose $\epsilon:=\frac{L B C}{C_{1}}$, where $C$ is the constant from (7.9). Noting that $M \preccurlyeq N$, the lemma follows.

Now we are ready to check that (10.2) is the desired extension of $F$. That $f$ defines a $C^{\infty}$-function on $\mathbb{R}^{n}$ with $j_{K}^{\infty} f=F$ can be seen as follows. Let us fix a point $a \in K$ and $\alpha \in \mathbb{N}^{n}$. Since $\Gamma_{m}(t) \rightarrow \infty$ as $t \rightarrow 0$, we have $|\alpha|<p(x)=2 \Gamma_{m}\left(L d_{K}(x)\right)$ if $x \in \mathbb{R}^{n} \backslash K$ is sufficiently close to $a$. Thus, as $x \rightarrow a$,

$$
\begin{aligned}
& \left|f^{(\alpha)}(x)-F^{\alpha}(a)\right| \\
& \leq\left|\left(f-T_{\hat{x}}^{p(x)} F\right)^{(\alpha)}(x)\right|+\left|\left(T_{\hat{x}}^{p(x)} F\right)^{(\alpha)}(x)-F^{\alpha}(\hat{x})\right|+\left|F^{\alpha}(\hat{x})-F^{\alpha}(a)\right| \\
& =O\left(h_{m}\left(L A_{4} d_{K}(x)\right)\right)+O\left(d_{K}(x)\right)+O(|\hat{x}-a|),
\end{aligned}
$$

by (8.2), (10.4), and (10.7). Hence $f^{(\alpha)}(x) \rightarrow F^{\alpha}(a)$ as $x \rightarrow a$. 
To get an extension in $\mathcal{E}^{\{N\}}\left(\mathbb{R}^{n}\right)$ we first observe that, for $x \in \mathbb{R}^{n} \backslash K$ with $d_{K}(x)<1, \alpha \in \mathbb{N}^{n}$, and a suitable constant $A=A(n, M, N)$,

$$
\left|f^{(\alpha)}(x)\right| \leq\left|\left(T_{\hat{x}}^{p(x)} F\right)^{(\alpha)}(x)\right|+\left|f^{(\alpha)}(x)-\left(T_{\hat{x}}^{p(x)} F\right)^{(\alpha)}(x)\right| \leq C(L A)^{|\alpha|+1} N_{|\alpha|}
$$

by (10.3) and (10.7), because $h_{m} \leq 1$. In view of (8.1), it suffices to multiply $f$ with a suitable cutoff function of class $\mathcal{E}^{\{N\}}$ with support in $\left\{x: d_{K}(x)<1\right\}$ in order to obtain an extension in $\mathcal{E}^{\{N\}}\left(\mathbb{R}^{n}\right)$.

Remark 10.7. The proof shows that for each $\rho>0$ there is an extension operator $\mathcal{E}_{\rho}^{M}(K) \rightarrow \mathcal{E}_{A \rho}^{N}\left(\mathbb{R}^{n}\right)$, for a suitable constant $A$, but the operator depends on $\rho$ (through $L$ and $\epsilon$ ).

There is a corresponding result in the Beurling case.

Theorem 10.8 ([27]). Let $M, N$ be strongly log-convex weight sequences, $M$ of moderate growth with $m_{k}^{1 / k} \rightarrow \infty$, and $N$ non-quasianalytic with $M \preccurlyeq N$. Then $(M, N)$ is admissible if and only if $\mathcal{E}^{(M)}(A) \subseteq j_{A}^{\infty} \mathcal{E}^{(N)}\left(\mathbb{R}^{n}\right)$ for all closed $A \subseteq \mathbb{R}^{n}$.

Let $(M, N)$ be a pair of admissible weight sequences and let $L$ be a non-negative sequence such that $L \triangleleft M$. Then there exists an admissible pair of weight sequences $\left(M^{\prime}, N^{\prime}\right)$ such that $L \preccurlyeq M^{\prime}$ and $N^{\prime} \triangleleft N$. Indeed, as in the proof of Lemma 8.5 there is a decreasing sequence $\left(\epsilon_{k}\right)_{k \geq 1}$ tending to 0 with $L_{k} \leq \epsilon_{1} \cdots \epsilon_{k} M_{k}$ for all $k$. Applying Lemma 3.4 to $\alpha_{k}=0, \beta_{k}=\max \left\{\epsilon_{k}, \frac{k}{\mu_{k}}\right\}$, and $\gamma_{k}=\frac{k}{\mu_{k}}$, gives a sequence $\left(\theta_{k}\right)_{k \geq 1}$ such that $\theta_{k} \nearrow \infty, \frac{k \theta_{k}}{\mu_{k}} \searrow 0$ and $\theta_{k} \epsilon_{k} \rightarrow 0$. Applying Lemma 3.4 once more, now to $\alpha_{k}=\frac{1}{\nu_{k}}, \beta_{k}=\max \left\{\frac{1}{\theta_{k}}, \frac{k}{\nu_{k}}\right\}$, and $\gamma_{k}=\frac{k}{\nu_{k}}$, yields a sequence $\left(\vartheta_{k}\right)_{k \geq 1}$ with $\vartheta_{k} \nearrow \infty$ such that

$$
\sum_{j \geq k} \frac{\vartheta_{j}}{\nu_{j}} \leq 8 \vartheta_{k} \sum_{j \geq k} \frac{1}{\nu_{j}}, \quad \text { for all } k \geq 1
$$

$\frac{\vartheta_{k}}{\theta_{k}} \rightarrow 0$, and $\frac{k \vartheta_{k}}{\nu_{k}} \searrow 0$. Define $\mu_{k}^{\prime}:=\frac{\mu_{k}}{\theta_{k}}$ and $\nu_{k}^{\prime}:=\frac{\nu_{k}}{\vartheta_{k}}$ for $k \geq 1$ and $\mu_{0}^{\prime}=\nu_{0}^{\prime}:=1$. Then $\frac{\mu_{k}^{\prime}}{k} \nearrow \infty$ and $\frac{\nu_{k}^{\prime}}{k} \nearrow \infty$ and hence $M_{k}^{\prime}:=\mu_{0}^{\prime} \mu_{1}^{\prime} \cdots \mu_{k}^{\prime}$ and $N_{k}^{\prime}:=\nu_{0}^{\prime} \nu_{1}^{\prime} \cdots \nu_{k}^{\prime}$ are strongly log-convex weight sequences such that $\left(m_{k}^{\prime}\right)^{1 / k} \rightarrow \infty$ and $\left(n_{k}^{\prime}\right)^{1 / k} \rightarrow \infty$. That $M^{\prime}$ has moderate growth follows from

$$
\frac{M_{j+k}^{\prime}}{M_{j}^{\prime} M_{k}^{\prime}}=\frac{M_{j+k}}{M_{j} M_{k}} \frac{\theta_{1} \cdots \theta_{j} \theta_{1} \cdots \theta_{k}}{\theta_{1} \cdots \theta_{j+k}} \leq \frac{M_{j+k}}{M_{j} M_{k}} .
$$

Furthermore,

$$
\sum_{j \geq k} \frac{1}{\nu^{\prime}}=\sum_{j \geq k} \frac{\vartheta_{j}}{\nu_{j}} \leq 8 \vartheta_{k} \sum_{j \geq k} \frac{1}{\nu_{j}} \lesssim \frac{k \vartheta_{k}}{\mu_{k}} \lesssim \frac{k \theta_{k}}{\mu_{k}}=\frac{k}{\mu_{k}^{\prime}}
$$

and hence $\left(M^{\prime}, N^{\prime}\right)$ is an admissible pair. We have $L \preccurlyeq M^{\prime}$ and $N^{\prime} \triangleleft N$, since $\left(\frac{L_{k}}{M_{k}^{\prime}}\right)^{1 / k}=\left(\frac{L_{k}}{M_{k}}\right)^{1 / k}\left(\theta_{1} \cdots \theta_{k}\right)^{1 / k} \leq\left(\theta_{1} \epsilon_{1} \cdots \theta_{k} \epsilon_{k}\right)^{1 / k}$ is bounded and $\left(\frac{N_{k}}{N_{k}^{\prime}}\right)^{1 / k}=$ $\left(\vartheta_{1} \cdots \vartheta_{k}\right)^{1 / k} \rightarrow \infty$.

Then it is easy to conclude $\mathcal{E}^{(M)}(A) \subseteq j_{A}^{\infty} \mathcal{E}^{(N)}\left(\mathbb{R}^{n}\right)$ for all closed $A \subseteq \mathbb{R}^{n}$, by a reduction argument to the Roumieu case (similar to the proof of Theorem 8.6). 
10.4. Extension operator. Concerning the existence of extension operators in the mixed setting we have

Theorem 10.9 ([27]). Let $M, N$ be strongly log-convex weight sequences, $M$ of moderate growth with $m_{k}^{1 / k} \rightarrow \infty$, and $N$ non-quasianalytic with $M \triangleleft N$. Then the following conditions are equivalent:

(1) $\lim _{k \rightarrow \infty} \frac{\mu_{k}}{k} \sum_{j \geq k} \frac{1}{\nu_{j}}=0$.

(2) $\Lambda^{\{M\}} \subseteq j_{\{0\}}^{\infty} \mathcal{E}^{(N)}\left(\mathbb{R}^{n}\right)$.

(3) There exists an extension operator $\mathcal{E}^{\{M\}}(A) \rightarrow \mathcal{E}^{(N)}\left(\mathbb{R}^{n}\right)$ for each closed non-empty subset $A \subseteq \mathbb{R}^{n}$.

We briefly comment on the implication $(1) \Rightarrow(3)$. The idea is to show that (1) implies the existence of an admissible pair $\left(M^{\prime}, N^{\prime}\right)$ of weight sequences such that $M \triangleleft M^{\prime} \triangleleft N^{\prime} \triangleleft N$. This can be achieved again with the help of Lemma 3.4; for the details we refer to [27, Proposition 20]. Then the extension operator is given by the composite

$$
\mathcal{E}^{\{M\}}(K) \hookrightarrow \mathcal{E}_{1}^{M^{\prime}}(K) \longrightarrow \mathcal{E}_{A}^{N^{\prime}}\left(\mathbb{R}^{n}\right) \hookrightarrow \mathcal{E}^{(N)}\left(\mathbb{R}^{n}\right)
$$

where the middle arrow is the extension operator from Remark 10.7.

Remark 10.10. All extensions in this section can be chosen to be analytic in the complement of $A$ in view of Theorem 8.9.

\section{Part 2. More general ultradifferentiable classes}

\section{Further ULtradifFERENTIABLE FUnCTIONS}

We introduce weight functions and Braun-Meise-Taylor classes. This quickly leads to the notion of the associated weight matrix which allows us to build the theory of Braun-Meise-Taylor classes (and of more general ultradifferentiable classes) to a great extent upon the theory of Denjoy-Carleman classes.

\subsection{Weight functions.}

Definition 11.1. A weight function is a continuous increasing function $\omega$ : $[0, \infty) \rightarrow[0, \infty)$ with $\omega(0)=0$ satisfying the following three properties:

(1) $\omega(2 t)=O(\omega(t))$ as $t \rightarrow \infty$.

(2) $\log (t)=o(\omega(t))$ as $t \rightarrow \infty$.

(3) $\varphi: t \mapsto \omega\left(e^{t}\right)$ is convex on $[0, \infty)$.

Note that (2) is equivalent to $\lim _{t \rightarrow \infty} \frac{t}{\varphi(t)}=0$ and implies $\lim _{t \rightarrow \infty} \omega(t)=\infty$. For each weight function $\omega$ there is a weight function $\tilde{\omega}$ which coincides with $\omega$ for all sufficiently large $t>0$ and such that $\left.\tilde{\omega}\right|_{[0,1]}=0$. It is hence no loss of generality to assume that $\left.\omega\right|_{[0,1]}=0$ which we shall do tacitly if convenient.

Since $\varphi$ is convex, increasing, and $\varphi(0)=0$, we may consider the Young conjugate $\varphi^{*}:[0, \infty) \rightarrow[0, \infty)$ of $\varphi$, given by

$$
\varphi^{*}(t):=\sup _{s \geq 0}(s t-\varphi(s)), \quad t \geq 0 .
$$

The function $\varphi^{*}$ is convex, increasing, and it satisfies $\varphi^{*}(0)=0, \varphi^{* *}=\varphi$ (here we use $\left.\left.\omega\right|_{[0,1]}=0\right)$, and $\lim _{t \rightarrow \infty} \frac{t}{\varphi^{*}(t)}=0$. Moreover, the functions $t \mapsto \frac{\varphi(t)}{t}$ and $t \mapsto \frac{\varphi^{*}(t)}{t}$ are increasing on $(0, \infty)$. 
11.2. Braun-Meise-Taylor classes. Let $\omega$ be a weight function. Let $U$ be an open subset of $\mathbb{R}^{n}$ and $\rho>0$. For $f \in C^{\infty}(U)$ and compact $K \subseteq U$ we consider the seminorm

$$
\|f\|_{K, \rho}^{\omega}:=\sup _{x \in K} \sup _{\alpha \in \mathbb{N}^{n}}\left|f^{(\alpha)}(x)\right| \exp \left(-\frac{1}{\rho} \varphi^{*}(\rho|\alpha|)\right) .
$$

(Occasionally, we will also use $\|f\|_{U, \rho}^{\omega}$ for open sets $U$.) We define the Braun-MeiseTaylor classes

$$
\begin{aligned}
& \mathcal{E}^{(\omega)}(U):=\left\{f \in C^{\infty}(U): \forall K \subseteq_{c p} U \forall \rho>0:\|f\|_{K, \rho}^{\omega}<\infty\right\}, \\
& \mathcal{E}^{\{\omega\}}(U):=\left\{f \in C^{\infty}(U): \forall K \subseteq_{c p} U \exists \rho>0:\|f\|_{K, \rho}^{\omega}<\infty\right\} .
\end{aligned}
$$

The class $\mathcal{E}^{(\omega)}(U)$ is said to be of Beurling type and the class $\mathcal{E}^{\{\omega\}}(U)$ of Roumieu type. In analogy to Denjoy-Carleman classes we use $\mathcal{E}^{[\omega]}(U)$ as placeholder for either $\mathcal{E}^{(\omega)}(U)$ or $\mathcal{E}^{\{\omega\}}(U)$; cf. Convention 2.1.

We endow the vector spaces $\mathcal{E}^{[\omega]}(U)$ with their natural locally convex topologies: $\mathcal{E}^{(\omega)}(U)$ is supplied with its natural Fréchet space topology and $\mathcal{E}^{\{\omega\}}(U)$ with the projective limit topology over $K$ of the inductive limit topology over $\rho$; note that it suffices to take countable limits. Then $\mathcal{E}^{(\omega)}(U)$ is a nuclear Fréchet space and $\mathcal{E}^{\{\omega\}}(U)$ is nuclear, complete, and reflexive; see [19, Proposition 4.9].

Remark 11.2. The classes $\mathcal{E}^{[\omega]}$ originate from work of Beurling [7] (see also Björck [10]). Beurling described his non-quasianalytic classes by decay properties of the Fourier transform of a compactly supported function:

$$
\begin{aligned}
& \mathcal{D}^{(\omega)}(U)=\left\{f \in C_{c}(U): \forall \rho>0: \int_{\mathbb{R}^{n}}|\widehat{f}(\xi)| e^{\rho \omega(\xi)} d t<\infty\right\}, \\
& \mathcal{D}^{\{\omega\}}(U)=\left\{f \in C_{c}(U): \exists \rho>0: \int_{\mathbb{R}^{n}}|\widehat{f}(\xi)| e^{\rho \omega(\xi)} d t<\infty\right\} .
\end{aligned}
$$

Here, and below, $\omega(\xi):=\omega(|\xi|)$. Braun, Meise, and Taylor [19] showed that these classes are non-trivial (for each non-empty open set $U \subseteq \mathbb{R}^{n}$ ) provided that $\omega$ is a weight function (not necessarily satisfying $11.1(3))^{13}$ such that

$$
\int_{1}^{\infty} \frac{\omega(t)}{t^{2}} d t<\infty
$$

We will give an independent proof of this fact in Theorem 11.17. Furthermore, if also the condition 11.1(3) is fulfilled, then the classes $\mathcal{D}^{[\omega]}$ also admit the description

$$
\begin{gathered}
\mathcal{D}^{(\omega)}(U)=\left\{f \in \mathcal{D}(U): \forall \rho>0:\|f\|_{U, \rho}^{\omega}<\infty\right\}, \\
\mathcal{D}^{\{\omega\}}(U)=\left\{f \in \mathcal{D}(U): \exists \rho>0:\|f\|_{U, \rho}^{\omega}<\infty\right\} .
\end{gathered}
$$

This was the starting point for the introduction of the local classes $\mathcal{E}^{[\omega]}(U)$ and the development of a corresponding theory of ultradistributions in [19].

11.3. Associated weight matrix. We associate with a weight function $\omega$ a oneparameter family $\mathfrak{W}=\left\{W^{x}\right\}_{x>0}$ of weight sequences $W^{x}=\left(W_{k}^{x}\right)$ defined by

$$
W_{k}^{x}:=\exp \left(\frac{1}{x} \varphi^{*}(x k)\right), \quad k \in \mathbb{N} .
$$

The family $\mathfrak{W}$ is called the associated weight matrix of $\omega$. As usual for weight sequences, we write

$$
W_{k}^{x}=\vartheta_{0}^{x} \vartheta_{1}^{x} \vartheta_{2}^{x} \cdots \vartheta_{k}^{x}=k ! w_{k}^{x} .
$$

\footnotetext{
${ }^{13}$ Beurling and Björck had additionally assumed that $\omega$ is subadditive.
} 
Notice that, for any $\rho>0$,

$$
\|f\|_{K, \rho}^{\omega}=\sup _{\alpha \in \mathbb{N}^{n}} \frac{\left\|f^{(\alpha)}\right\|_{K}}{\exp \left(\frac{1}{\rho} \varphi^{*}(\rho|\alpha|)\right)}=\sup _{\alpha \in \mathbb{N}^{n}} \frac{\left\|f^{(\alpha)}\right\|_{K}}{W_{|\alpha|}^{\rho}}=\|f\|_{K, 1}^{W^{\rho}} .
$$

Lemma 11.3. We have:

(1) Each $W^{x}$ is a weight sequence.

(2) $\vartheta^{x} \leq \vartheta^{y}$ if $x \leq y$, which entails $W^{x} \leq W^{y}$.

(3) For all $x>0$ and all $j, k \in \mathbb{N}, W_{j+k}^{x} \leq W_{j}^{2 x} W_{k}^{2 x}$ and $w_{j+k}^{x} \leq w_{j}^{2 x} w_{k}^{2 x}$.

(4) For all $x>0$ and all $k \in \mathbb{N}_{\geq 2}, \vartheta_{2 k}^{x} \leq \vartheta_{k}^{4 x}$.

(5) $\forall \rho>0 \exists H \geq 1 \forall x>0 \exists C \geq 1 \forall k \in \mathbb{N}: \rho^{k} W_{k}^{x} \leq C W_{k}^{H x}$.

Proof. (1) The convexity of $\varphi^{*}$ implies that each $\vartheta^{x}$ is increasing. And $\left(W_{k}^{x}\right)^{1 / k} \nearrow$ $\infty$ follows from $t \mapsto \frac{\varphi^{*}(t)}{t} \nearrow \infty$

(2) Let $0<x \leq y$. Then

$$
\vartheta_{k}^{x}=\exp \left(\frac{\varphi^{*}(x k)-\varphi^{*}(x(k-1))}{x}\right) \leq \exp \left(\frac{\varphi^{*}(y k)-\varphi^{*}(y(k-1))}{y}\right)=\vartheta_{k}^{y}
$$

again by the convexity of $\varphi^{*}$.

(3) The convexity of $\varphi^{*}$ and $\varphi^{*}(0)=0$ implies

$$
\varphi^{*}(t)+\varphi^{*}(s) \leq \varphi^{*}(t+s) \leq \frac{1}{2} \varphi^{*}(2 t)+\frac{1}{2} \varphi^{*}(2 s), \quad t, s \geq 0 .
$$

As a consequence

$$
W_{j}^{x} W_{k}^{x} \leq W_{j+k}^{x} \leq W_{j}^{2 x} W_{k}^{2 x}, \quad j, k \in \mathbb{N},
$$

and equivalently

$$
w_{j}^{x} w_{k}^{x} \leq\left(\begin{array}{c}
j+k \\
j
\end{array}\right) w_{j+k}^{x} \leq w_{j}^{2 x} w_{k}^{2 x}, \quad j, k \in \mathbb{N}
$$

(4) The inequality $\vartheta_{2 k}^{x} \leq \vartheta_{k}^{4 x}$ is equivalent to

$$
\frac{\varphi^{*}(2 k x)-\varphi^{*}((2 k-1) x)}{x} \leq \frac{\varphi^{*}(4 k x)-\varphi^{*}(4(k-1) x)}{4 x},
$$

which follows from the convexity of $\varphi^{*}$ if $k \geq 2$.

(5) The statement follows from the following inequality

$$
\exists L \geq 1 \forall t \geq 0 \forall s \in \mathbb{N}: L^{s} \varphi^{*}(t)+s L^{s} t \leq \varphi^{*}\left(L^{s} t\right)+\sum_{i=1}^{s} L^{i}
$$

if we choose $s$ such that $e^{s} \geq \sigma$ and set $t:=\rho k, H:=L^{s}$ and $C:=\exp \left(\frac{1}{H \rho} \sum_{i=1}^{s} L^{i}\right)$. By 11.1(1), there exists $L_{1} \geq 1$ such that $\omega(2 t) \leq L_{1} \omega(t)+L_{1}$ for all $t \geq 0$ and hence there is $L \geq 1$ such that $\varphi(t+1) \leq L \varphi(t)+L$ for all $t \geq 0$. Thus, for $t \geq 0$,

$$
\varphi^{*}(L t)+L=\sup _{s \geq 0}(L t s-(\varphi(s)-L)) \geq \sup _{s \geq 1}(L t s-L \varphi(s-1))=L \varphi^{*}(t)+L t,
$$

and the desired inequality follows by iteration. 


\subsection{Weight matrix description of Braun-Meise-Taylor classes.}

Theorem 11.4 ([74]). Let $\omega$ be a weight function with associated weight matrix $\mathfrak{W}=\left\{W^{x}\right\}_{x>0}$. Let $U \subseteq \mathbb{R}^{n}$ be open and $K \subseteq U$ compact.

(1) For each $x>0$ we have the continuous inclusions $\mathcal{E}^{\left\{W^{x}\right\}}(U) \subseteq \mathcal{E}^{\{\omega\}}(U)$ and $\mathcal{E}^{(\omega)}(U) \subseteq \mathcal{E}^{\left(W^{x}\right)}(U)$.

(2) As locally convex spaces

$$
\begin{aligned}
& \mathcal{E}^{(\omega)}(K)=\operatorname{proj}_{x>0} \mathcal{E}^{\left(W^{x}\right)}(K)=\operatorname{proj}_{x>0} \operatorname{proj}_{\rho>0} \mathcal{E}_{\rho}^{W^{x}}(K), \\
& \mathcal{E}^{\{\omega\}}(K)=\operatorname{ind}_{x>0} \mathcal{E}^{\left\{W^{x}\right\}}(K)=\operatorname{ind}_{x>0} \operatorname{ind}_{\rho>0} \mathcal{E}_{\rho}^{W^{x}}(K), \\
& \mathcal{E}^{(\omega)}(U)=\operatorname{proj}_{K \subseteq_{c p} U} \operatorname{proj}_{x>0} \mathcal{E}^{\left(W^{x}\right)}(K)=\operatorname{proj}_{x>0} \mathcal{E}^{\left(W^{x}\right)}(U), \\
& \mathcal{E}^{\{\omega\}}(U)=\operatorname{proj}_{K \subseteq_{c p} U} \operatorname{ind}_{x>0} \mathcal{E}^{\left\{W^{x}\right\}}(K) .
\end{aligned}
$$

Proof. (1) Let $x>0$ be fixed. If $f \in \mathcal{E}^{\left\{W^{x}\right\}}(U)$, then for each compact subset $K \subseteq U$ there exists $\rho>0$ such that $\|f\|_{K, \rho}^{W^{x}}<\infty$. By Lemma 11.3(5) and (11.2), there exist constants $H, C \geq 1$ such that

$$
\infty>C\|f\|_{K, \rho}^{W^{x}} \geq\|f\|_{K, 1}^{W^{H x}}=\|f\|_{K, H x}^{\omega},
$$

whence $f \in \mathcal{E}^{\{\omega\}}(U)$.

Assume that $f \in \mathcal{E}^{(\omega)}(U)$. Let $x>0$ and $\rho>0$ be fixed. By Lemma 11.3(5), there exist constants $H, C \geq 1$ such that $W_{k}^{x} \leq C \rho^{k} W_{k}^{H x}$ for all $k$. For each compact subset $K \subseteq U$ we have $\|f\|_{K, \frac{x}{H}}^{\omega}<\infty$, and, thus, using (11.2),

$$
\infty>C\|f\|_{K, \frac{x}{H}}^{\omega}=C\|f\|_{K, 1}^{W \frac{x}{H}} \geq\|f\|_{K, \rho}^{W^{x}} .
$$

Since $\rho>0$ was arbitrary, we may conclude that $f \in \mathcal{E}^{\left(W^{x}\right)}(U)$.

(2) now follows from (1), since we have the continuous inclusions $\mathcal{E}^{(\omega)}(K) \supseteq$ $\operatorname{proj}_{x>0} \mathcal{E}^{\left(W^{x}\right)}(K)$ and $\mathcal{E}^{\{\omega\}}(K) \subseteq \operatorname{ind}_{x>0} \mathcal{E}^{\left\{W^{x}\right\}}(K)$ by definition.

Corollary 11.5. Let $\omega$ be a weight function. Then:

(1) $\mathcal{E}^{[\omega]}(U)$ is a ring.

(2) If $C^{\omega}(U) \subseteq \mathcal{E}^{[\omega]}(U)$ and $\psi: \mathbb{R}^{m} \supseteq V \rightarrow U$ is real analytic, then the pullback $\psi^{*}: \mathcal{E}^{[\omega]}(U) \rightarrow \mathcal{E}^{[\omega]}(V)$ is well-defined.

(3) $\mathcal{E}^{[\omega]}(U)$ is stable under differentiation.

Proof. (1) and (2) follow from the fact that each $W^{x}$ is a weight sequence; cf. Section 2.5. (3) is a consequence of Lemma 11.3(3).

11.5. Braun-Meise-Taylor vs. Denjoy-Carleman classes. Theorem 11.4 shows that every Braun-Meise-Taylor class can be represented as a union or an intersection of Denjoy-Carleman classes. The question arises as to when BraunMeise-Taylor and Denjoy-Carleman classes coincide.

Theorem 11.6. Let $\omega$ be a weight function with associated weight matrix $\mathfrak{W}=$ $\left\{W^{x}\right\}_{x>0}$. Let $U \subseteq \mathbb{R}^{n}$ be open and non-empty. The following conditions are equivalent:

(1) $\exists H \geq 1 \forall t \geq 0: 2 \omega(t) \leq \omega(H t)+H$.

(2) The weight sequences $W^{x}$ and $W^{y}$ are equivalent for all $x, y>0$.

(3) $\mathcal{E}^{\{\omega\}}(U)=\mathcal{E}^{\left\{W^{x}\right\}}(U)$ for all $x>0$.

(4) $\mathcal{E}^{(\omega)}(U)=\mathcal{E}^{\left(W^{x}\right)}(U)$ for all $x>0$. 
(5) $W^{x}$ has moderate growth for some (equivalently, for all) $x>0$.

The equivalence of (1) and (2) can be seen by straightforward computations. Then $(2) \Leftrightarrow(3) \Leftrightarrow(4)$ follow from Theorem 11.4. For the equivalence with (5) see [74]. Conversely, one may ask when for a given weight sequence $M$ there exists a weight function $\omega$ such that $\mathcal{E}^{[M]}(U)=\mathcal{E}^{[\omega]}(U)$.

Theorem 11.7 ([16, Theorem 14]). Let $M=\left(M_{k}\right)$ be a derivation-closed weight sequence with $m_{k}^{1 / k} \rightarrow \infty$. The following conditions are equivalent:

(1) There exists a weight function $\omega$ such that for all open $U \subseteq \mathbb{R}^{n}$ we have $\mathcal{E}^{[\omega]}(U)=\mathcal{E}^{[M]}(U)$ as vector spaces and/or as locally convex spaces.

(2) The weight sequence $M$ has moderate growth and satisfies

$$
\exists Q \in \mathbb{N}_{\geq 1}: \liminf _{k \rightarrow \infty} \frac{\mu_{Q k}}{\mu_{k}}>1 .
$$

(3) The weight sequence $M$ has moderate growth, the associated function $\omega_{M}(t)=\sup _{k \in \mathbb{N}} \log \frac{t^{k}}{M_{k}}$ is a weight function, and (1) holds with $\omega=\omega_{M}$.

Example 11.8. (1) Let $s \geq 1$. Then $\gamma_{s}(t):=\max \left\{0, t^{\frac{1}{s}}-1\right\}$ is a weight function and we have the identity

$$
\mathcal{E}^{\left[\gamma_{s}\right]}(U)=\mathcal{E}^{\left[G^{s}\right]}(U)
$$

where $G^{s}$ denotes the Gevrey sequence $G_{k}^{s}=k !^{s}$. See also Example 12.3.

(2) For $s>1, \omega_{s}(t):=\max \left\{0,(\log t)^{s}\right\}$ is a weight function with associated weight matrix $\mathfrak{W}^{s}=\left\{W^{s, x}\right\}_{x>0}$ given by $W_{k}^{s, x}=\exp \left(C_{s} x^{1 /(s-1)} k^{s /(s-1)}\right)$. The classes $\mathcal{E}^{\left[\omega_{s}\right]}$ are not Denjoy-Carleman classes.

11.6. Weight matrices and associated ultradifferentiable classes. The weight matrix description of Braun-Meise-Taylor classes motivates the definition of ultradifferentiable classes by general weight matrices, providing a general framework for ultradifferentiability.

Definition 11.9. A weight matrix is by definition a family $\mathfrak{M}$ of weight sequences $M \in \mathfrak{M}$ which is totally ordered with respect to the natural order relation on sequences (i.e., for all $M, N \in \mathfrak{M}$ we have $M \leq N$ or $N \leq M$ ).

Let $\mathfrak{M}$ be a weight matrix. Let $U \subseteq \mathbb{R}^{n}$ open and $K \subseteq U$ compact. We define

$$
\begin{aligned}
\mathcal{E}^{\{\mathfrak{M}\}}(K) & :=\bigcup_{M \in \mathfrak{M}} \mathcal{E}^{\{M\}}(K), \\
\mathcal{E}^{(\mathfrak{M})}(K) & :=\bigcap_{M \in \mathfrak{M}} \mathcal{E}^{(M)}(K), \\
\mathcal{E}^{\{\mathfrak{M}\}}(U) & :=\bigcap_{K \subseteq_{c p} U} \bigcup_{M \in \mathfrak{M}} \mathcal{E}^{\{M\}}(K)=\bigcap_{K \subseteq_{c p} U} \mathcal{E}^{\{\mathfrak{M}\}}(K), \\
\mathcal{E}^{(\mathfrak{M})}(U) & :=\bigcap_{K \subseteq c p} \bigcap_{M \in \mathfrak{M}} \mathcal{E}^{(M)}(K)=\bigcap_{K \subseteq_{c p} U} \mathcal{E}^{(\mathfrak{M})}(K)=\bigcap_{M \in \mathfrak{M}} \mathcal{E}^{(M)}(U)
\end{aligned}
$$

and endow these spaces with their natural locally convex topologies. The class $\mathcal{E}^{(\mathfrak{M})}(U)$ is said to be of Beurling type and the class $\mathcal{E}^{\{\mathfrak{M}\}}(U)$ of Roumieu type. Again we use $\mathcal{E}^{[\mathfrak{M}]}(U)$ as placeholder for either $\mathcal{E}^{(\mathfrak{M})}(U)$ or $\mathcal{E}^{\{\mathfrak{M}\}}(U)$.

For each weight matrix $\mathfrak{M}$ there exists a countable weight matrix $\mathfrak{M}^{\prime}$ such that $\mathcal{E}^{[\mathfrak{M}]}(U)=\mathcal{E}^{\left[\mathfrak{M}^{\prime}\right]}(U)$ as locally convex spaces; see [40, Lemma 2.5]. 
Note that all $\mathcal{E}^{[\mathfrak{M}]}(U)$ are rings and if $C^{\omega}(U) \subseteq \mathcal{E}^{[\mathfrak{M}]}(U)$ then the class $\mathcal{E}^{[\mathfrak{M}]}$ is stable by analytic change of coordinates.

11.7. Inclusion relations. The inclusion relations for the classes $\mathcal{E}^{[\mathfrak{M}]}$ follow easily from those for Denjoy-Carleman classes. For weight matrices $\mathfrak{M}$ and $\mathfrak{N}$ we define

$$
\begin{array}{cll}
\mathfrak{M}(\preccurlyeq) \mathfrak{N} & : \Leftrightarrow & \forall N \in \mathfrak{N} \exists M \in \mathfrak{M}: M \preccurlyeq N, \\
\mathfrak{M}\{\preccurlyeq\} \mathfrak{N} & : \Leftrightarrow & \forall M \in \mathfrak{M} \exists N \in \mathfrak{N}: M \preccurlyeq N, \\
\mathfrak{M}(\preccurlyeq\} \mathfrak{N} & : \Leftrightarrow \quad \exists M \in \mathfrak{M} \exists N \in \mathfrak{N}: M \preccurlyeq N, \\
\mathfrak{M}\{\triangleleft) \mathfrak{N} & : \Leftrightarrow & \forall M \in \mathfrak{M} \forall N \in \mathfrak{N}: M \triangleleft N .
\end{array}
$$

Moreover, we set

$$
\mathfrak{M}[\approx] \mathfrak{N} \quad: \Leftrightarrow \quad \mathfrak{M}[\preccurlyeq] \mathfrak{N}[\preccurlyeq] \mathfrak{M} .
$$

Lemma $11.10([74])$. Let $\mathfrak{M}$ and $\mathfrak{N}$ be two weight matrices. Let $U \subseteq \mathbb{R}^{n}$ be open and non-empty. Then:

(1) $\mathfrak{M}[\preccurlyeq] \mathfrak{N}$ if and only if $\mathcal{E}^{[\mathfrak{M}]}(U) \subseteq \mathcal{E}^{[\mathfrak{N}]}(U)$.

(2) $\mathfrak{M}(\preccurlyeq\} \mathfrak{N}$ if and only if $\mathcal{E}^{(\mathfrak{M})}(U) \subseteq \mathcal{E}^{\{\mathfrak{N}\}}(U)$.

(3) $\mathfrak{M}\{\triangleleft) \mathfrak{N}$ if and only if $\mathcal{E}^{\{\mathfrak{M}\}}(U) \subseteq \mathcal{E}^{(\mathfrak{N})}(U)$.

All inclusions are continuous. For the "if" part it suffices to have the inclusions on $U \subseteq \mathbb{R}$

Corollary 11.11. A class of Roumieu type and a class of Beurling type can never coincide.

Proof. Observe that $\mathfrak{M}(\preccurlyeq\} \mathfrak{N}\{\triangleleft) \mathfrak{M}$ is impossible for any two weight matrices $\mathfrak{M}$ and $\mathfrak{N}$ and apply Lemma 11.10.

In the special case of Braun-Meise-Taylor classes we obtain

Proposition 11.12. Let $\omega$ and $\sigma$ be weight functions. Let $U \subseteq \mathbb{R}^{n}$ be open and non-empty. Then:

(1) $\sigma(t)=O(\omega(t))$ as $t \rightarrow \infty$ if and only if $\mathcal{E}^{[\omega]}(U) \subseteq \mathcal{E}^{[\sigma]}(U)$.

(2) $\sigma(t)=o(\omega(t))$ as $t \rightarrow \infty$ if and only if $\mathcal{E}^{\{\omega\}}(U) \subseteq \mathcal{E}^{(\sigma)}(U)$.

(3) $\sigma(t)=O(\omega(t))$ as $t \rightarrow \infty$ if and only if $\mathcal{E}^{(\omega)}(U) \subseteq \mathcal{E}^{\{\sigma\}}(U)$.

All inclusions are continuous. For the "if" part it suffices to have the inclusions on $U \subseteq \mathbb{R}$

Note that $\omega$ and $\sigma$ are said to be equivalent if $\sigma(t)=O(\omega(t))$ and $\omega(t)=O(\sigma(t))$ as $t \rightarrow \infty$.

Proof. Let $\mathfrak{W}=\left\{W^{x}\right\}_{x>0}$ and $\mathfrak{S}=\left\{S^{x}\right\}_{x>0}$ be the associated weight matrices of $\omega$ and $\sigma$, respectively. Let us prove (1); (2) and (3) can be shown similarly. In view of Lemma 11.10 it suffices to prove

$$
\sigma(t)=O(\omega(t)) \text { as } t \rightarrow \infty \text { if and only if } \mathfrak{W}[\preccurlyeq] \mathfrak{S} \text {. }
$$

Assume that $\sigma(t)=O(\omega(t))$ as $t \rightarrow \infty$. Then there exists $H \geq 1$ such that $\sigma(t) \leq H \omega(t)+H$ for all $t \geq 0$. This implies $H \varphi_{\omega}^{*}(t) \leq \varphi_{\sigma}^{*}(H t)+H$ for the Young conjugates of $t \mapsto \sigma\left(e^{t}\right)$ and $t \mapsto \omega\left(e^{t}\right)$. In particular, for $t=x k$ we find

$$
W_{k}^{x}=\exp \left(\frac{1}{x} \varphi_{\omega}^{*}(x k)\right) \leq \exp \left(\frac{1}{H x} \varphi_{\sigma}^{*}(H x k)+\frac{1}{x}\right)=e^{1 / x} S_{k}^{H x} .
$$


That means there is $H \geq 1$ such that for all $x>0$ we have $W^{x} \leq e^{1 / x} S^{H x}$ which implies $\mathfrak{W}(\preccurlyeq) \mathfrak{S}$ as well as $\mathfrak{W}\{\preccurlyeq\} \mathfrak{S}$.

For the converse direction we assume $\mathfrak{W}\{\preccurlyeq\} \mathfrak{S}$; for the assumption $\mathfrak{W}(\preccurlyeq) \mathfrak{S}$ the arguments are analogous. This means, using Lemma 11.3(5),

$$
\forall x>0 \exists y>0 \exists C>0 \forall k \in \mathbb{N}: \frac{1}{x} \varphi_{\omega}^{*}(x k) \leq \frac{1}{y} \varphi_{\sigma}^{*}(y k)+C .
$$

By the convexity of $\varphi_{\omega}^{*}$ and $\varphi_{\sigma}^{*}$ we may conclude that

$$
\forall x>0 \exists y>0 \exists D>0 \forall t \geq 0: \frac{1}{x} \varphi_{\omega}^{*}(x t) \leq \frac{1}{y} \varphi_{\sigma}^{*}(y t)+C
$$

(for different $y$ and $C$ ). Then

$$
\frac{1}{y} \varphi_{\sigma}(t)=\sup _{s \geq 0}\left(t s-\frac{1}{y} \varphi_{\sigma}^{*}(y s)\right) \leq \sup _{s \geq 0}\left(t s-\frac{1}{x} \varphi_{\omega}^{*}(x s)\right)+C=\frac{1}{x} \varphi_{\omega}(t)+C,
$$

that is

$$
\frac{1}{y} \sigma(t) \leq \frac{1}{x} \omega(t)+C
$$

which implies $\sigma(t)=O(\omega(t))$ as $t \rightarrow \infty$.

Corollary 11.13. Let $\omega$ be a weight function. Let $U \subseteq \mathbb{R}^{n}$ be open and non-empty.

(1) $\mathcal{H}\left(\mathbb{C}^{n}\right) \subseteq \mathcal{E}^{(\omega)}(U)$ if and only if $\omega(t)=O(t)$ as $t \rightarrow \infty$.

(2) $C^{\omega}(U) \subseteq \mathcal{E}^{(\omega)}(U)$ if and only if $\omega(t)=o(t)$ as $t \rightarrow \infty$.

(3) $C^{\omega}(U) \subseteq \mathcal{E}^{\{\omega\}}(U)$ if and only if $\omega(t)=O(t)$ as $t \rightarrow \infty$.

Proof. This follows from Example 11.8(1) and Proposition 11.12.

11.8. Intersection and union of all non-quasianalytic Gevrey classes. Let $\mathfrak{G}:=\left\{G^{s}\right\}_{s>1}$ be the weight matrix consisting of all non-quasianalytic Gevrey sequences $G_{k}^{s}=k !^{s}$. It turns out that $\mathcal{E}^{(\mathfrak{G})}$ and $\mathcal{E}^{\{\mathfrak{G}\}}$ are neither Denjoy-Carleman nor Braun-Meise-Taylor classes.

Theorem 11.14 ([74]). The weight matrix $\mathfrak{G}=\left\{G^{s}\right\}_{s>1}$ has the following properties:

(1) We have $L \triangleleft G^{s} \triangleleft G^{t}$ for all $1<s<t$, where $L=\left(L_{k}\right)$ is the weight sequence $L_{k}:=k^{k}(\log (k+e))^{2 k}$.

(2) Let $U \subseteq \mathbb{R}^{n}$ be open and $K \subseteq U$ compact. As locally convex spaces

$$
\begin{aligned}
\mathcal{E}^{(\mathfrak{G})}(K) & =\operatorname{proj}_{s>1} \mathcal{E}^{\left(G^{s}\right)}(K)=\operatorname{proj}_{s>1} \mathcal{E}^{\left\{G^{s}\right\}}(K), \\
\mathcal{E}^{\{\mathfrak{G}\}}(K) & =\operatorname{ind}_{s>1} \mathcal{E}^{\left\{G^{s}\right\}}(K)=\operatorname{ind}_{s>1} \mathcal{E}^{\left(G^{s}\right)}(K), \\
\mathcal{E}^{(\mathfrak{G})}(U) & =\operatorname{proj}_{s>1} \mathcal{E}^{\left(G^{s}\right)}(U)=\operatorname{proj}_{s>1} \mathcal{E}^{\left\{G^{s}\right\}}(U), \\
\mathcal{E}^{\{\mathfrak{G}\}}(U) & =\operatorname{proj}_{K \subseteq_{c p} U} \operatorname{ind}_{s>1} \mathcal{E}^{\left\{G^{s}\right\}}(K)=\operatorname{proj}_{K \subseteq_{c p} U} \operatorname{ind}_{s>1} \mathcal{E}^{\left(G^{s}\right)}(K) .
\end{aligned}
$$

$(3) \mathcal{E}^{(\mathfrak{G})}$ and $\mathcal{E}^{\{\mathfrak{G}\}}$ are non-quasianalytic.

(4) Neither $\mathcal{E}^{(\mathfrak{G})}$ nor $\mathcal{E}^{\{\mathfrak{G}\}}$ coincides with a Denjoy-Carleman or a BraunMeise-Taylor class (as sets).

Proof. (1) If $1<s<t$, then

$$
\left(\frac{G_{k}^{s}}{G_{k}^{t}}\right)^{1 / k}=k !^{\frac{s-t}{k}} \leq k^{s-t} \rightarrow 0
$$

and

$$
\left(\frac{L_{k}}{G_{k}^{s}}\right)^{1 / k}=\frac{k}{k ! !^{s / k}}(\log (k+e))^{2} \leq e k^{1-s}(\log (k+e))^{2} \rightarrow 0 .
$$


(2) This follows from the definition and from the fact that $G^{s} \triangleleft G^{t}$ if $s<t$.

(3) is a consequence of (1), since the weight sequence $L$ is non-quasianalytic. It also follows from Theorem 11.16 .

(4) Suppose that there is a weight sequence $M$ such that $\mathcal{E}^{(M)}(\mathbb{R})=\mathcal{E}^{(\mathfrak{G})}(\mathbb{R})$. Then Lemma 11.10 implies $M(\preccurlyeq) \mathfrak{G}(\preccurlyeq) M$, i.e., $M \preccurlyeq G^{s}$ for all $s>1$ and there exists $t>1$ such that $G^{t} \preccurlyeq M$. Thus $M$ is equivalent to $G^{t}$ and hence

$$
\mathcal{E}^{(M)}(\mathbb{R})=\mathcal{E}^{\left(G^{t}\right)}(\mathbb{R}) \supsetneq \mathcal{E}^{(\mathfrak{G})}(\mathbb{R})=\mathcal{E}^{(M)}(\mathbb{R}),
$$

a contradiction. Similarly, if $\mathcal{E}^{\{M\}}(\mathbb{R})=\mathcal{E}^{\{\mathfrak{G}\}}(\mathbb{R})$ for some weight sequence $M$, then we conclude $M\{\preccurlyeq\} \mathfrak{G}\{\preccurlyeq\} M$, i.e., $M \preccurlyeq G^{s}$ for some $s>1$ and $G^{t} \preccurlyeq M$ for all $t>1$. So $M$ is equivalent to $G^{s}$ which leads to the contradiction

$$
\mathcal{E}^{\{M\}}(\mathbb{R})=\mathcal{E}^{\left\{G^{s}\right\}}(\mathbb{R}) \subsetneq \mathcal{E}^{\{\mathfrak{G}\}}(\mathbb{R})=\mathcal{E}^{\{M\}}(\mathbb{R}) .
$$

Now assume that there exists a weight function $\omega$ such that $\mathcal{E}^{(\omega)}(\mathbb{R})=\mathcal{E}^{(\mathfrak{G})}(\mathbb{R})$. Let $\mathfrak{W}=\left\{W^{x}\right\}_{x>0}$ be the weight matrix associated with $\omega$. Then $\mathfrak{W}(\preccurlyeq) \mathfrak{G}(\preccurlyeq) \mathfrak{W}$, i.e., for all $t>1$ there exists $x>0$ such that $W^{x} \preccurlyeq G^{t}$ and for all $y>0$ there exists $s>1$ such that $G^{s} \preccurlyeq W^{y}$. It follows that

$$
\forall y>0 \exists s>1 \exists x>0: W^{x} \preccurlyeq G^{s} \preccurlyeq W^{y} .
$$

As a consequence $\mathcal{E}^{\left[W^{x}\right]}(\mathbb{R}) \subseteq \mathcal{E}^{\left[G^{s}\right]}(\mathbb{R}) \subseteq \mathcal{E}^{\left[W^{y}\right]}(\mathbb{R})$. But, by Example 11.8(1), we have $\mathcal{E}^{\left[G^{s}\right]}(\mathbb{R})=\mathcal{E}^{\left[\gamma_{s}\right]}(\mathbb{R})$, where $\gamma_{s}(t)=t^{1 / s}$. Consequently,

$$
\mathcal{E}^{(\omega)}(\mathbb{R}) \subseteq \mathcal{E}^{\left(W^{x}\right)}(\mathbb{R}) \subseteq \mathcal{E}^{\left(G^{s}\right)}(\mathbb{R})=\mathcal{E}^{\left(\gamma_{s}\right)}(\mathbb{R})
$$

and thus $\gamma_{s}(t)=O(\omega(t))$ as $t \rightarrow \infty$, by Proposition 11.12. On the other hand,

$$
\mathcal{E}^{\left\{\gamma_{s}\right\}}(\mathbb{R})=\mathcal{E}^{\left\{G^{s}\right\}}(\mathbb{R}) \subseteq \mathcal{E}^{\left\{W^{y}\right\}}(\mathbb{R}) \subseteq \mathcal{E}^{\{\omega\}}(\mathbb{R})
$$

and hence $\omega(t)=O\left(\gamma_{s}(t)\right)$ as $t \rightarrow \infty$. But this leads to a contradiction:

$$
\mathcal{E}^{\left(G^{s}\right)}(\mathbb{R})=\mathcal{E}^{\left(\gamma_{s}\right)}(\mathbb{R})=\mathcal{E}^{(\omega)}(\mathbb{R})=\mathcal{E}^{(\mathfrak{G})}(\mathbb{R}) \subsetneq \mathcal{E}^{\left(G^{s}\right)}(\mathbb{R}) .
$$

In analogy, one shows that $\mathcal{E}^{\{\omega\}}(\mathbb{R})=\mathcal{E}^{\{\mathfrak{G}\}}(\mathbb{R})$ for some weight function $\omega$ is impossible.

In view of Corollary 11.11 the proof of (4) is complete.

Remark 11.15. The classes $\mathcal{E}^{[\mathfrak{G}]}$ enjoy good properties that the single Gevrey classes do not have, as investigated in [28]. In fact, if $M$ is a strongly logconvex weight sequence of moderate growth such that the "power" $M^{(a)}:=\left(k ! m_{k}^{a}\right)$ is non-quasianalytic for every $a>0$ (e.g. strongly regular sequences or $M_{k}:=$ $k ! \exp \left(\sum_{j=1}^{k}(\log j)^{\delta}\right)$ for $\left.\delta \in(0,1]\right)$, then the class $\bigcap_{a>0} \mathcal{B}^{\left\{M^{(a)}\right\}}$ admits versions of

(1) Whitney's extension theorem,

(2) Łojasiewicz's theorem on regularly situated compact sets,

(3) Weierstrass' division and preparation theorems,

(4) Whitney's spectral theorem.

Let us give a short argument for (1): for any jet $F$ of class $\bigcap_{a>0} \mathcal{B}^{\left\{M^{(a)}\right\}}$ on a compact set $K$ one can find a strongly log-convex non-quasianalytic weight sequence $L$ of moderate growth such that $L \triangleleft M^{(a)}$ for all $a>0$ and $F$ is of class $\mathcal{E}^{\{L\}}$; see [28, Proposition 5]. Then, if $L_{k}=k ! \ell_{k}$ and $\lambda_{k}^{*}:=\frac{\ell_{k}}{\ell_{k-1}}$, we find

$$
\sum_{j \geq k} \frac{1}{j\left(\lambda_{j}^{*}\right)^{2}} \leq \frac{1}{\lambda_{k}^{*}} \sum_{j \geq k} \frac{1}{j \lambda_{j}^{*}} \lesssim \frac{1}{\lambda_{k}^{*}},
$$


and hence $F$ has an extension $f$ in $\mathcal{B}^{\left\{L^{(2)}\right\}}\left(\mathbb{R}^{n}\right)$, by Theorem 10.4. But

$$
\left(\frac{L_{k}^{(2)}}{M_{k}^{(a)}}\right)^{1 / k}=\left(\frac{\ell_{k}^{2}}{m_{k}^{a}}\right)^{1 / k}=\left(\frac{\ell_{k}}{m_{k}^{a / 2}}\right)^{2 / k}=\left(\frac{L_{k}}{M_{k}^{(a / 2)}}\right)^{2 / k} \rightarrow 0,
$$

for all $a>0$ so that $f \in \bigcap_{a>0} \mathcal{B}^{\left\{M^{(a)}\right\}}$.

11.9. Quasianalyticity and non-quasianalyticity. The weight matrix description of Braun-Meise-Taylor classes, i.e. Theorem 11.4, allows us to deduce the characterization of (non-)quasianalyticity from the Denjoy-Carleman theorem 3.6. For a different proof relying on Hörmander's $L^{2}$-method and a Paley-Wiener theorem see [19].

Theorem 11.16. Let $\mathfrak{M}$ be a weight matrix. Then:

(1) $\mathcal{E}^{\{\mathfrak{M}\}}$ is quasianalytic if and only if all $M \in \mathfrak{M}$ are quasianalytic.

(2) $\mathcal{E}^{(\mathfrak{M})}$ is quasianalytic if and only if some $M \in \mathfrak{M}$ is quasianalytic.

Proof. (1) If some $M \in \mathfrak{M}$ is non-quasianalytic, then there exists a non-trivial function with compact support of class $\mathcal{E}^{\{M\}}$, thus of of class $\mathcal{E}^{\{\mathfrak{M}\}}$. Conversely,

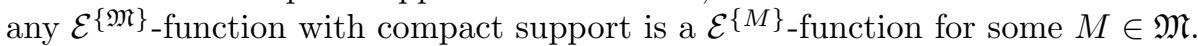

(2) If some $M \in \mathfrak{M}$ is quasianalytic, then clearly $\mathcal{E}^{(\mathfrak{M})}$ is quasianalytic since $\mathcal{E}^{(\mathfrak{M})} \subseteq \mathcal{E}^{(M)}$. Conversely, assume that all $M \in \mathfrak{M}$ are non-quasianalytic. We may assume that the weight matrix $\mathfrak{M}$ is countable $([40$, Lemma 2.5$])$, i.e., $\mathfrak{M}=$ $\left\{M^{n}\right\}_{n \in \mathbb{N}}$, where $M^{n} \geq M^{n+1}$ for all $n$. Set $\alpha_{k}^{n}:=\left(M_{k}^{n}\right)^{-1 / k}$. Then, for each $n$ the sequence $\alpha^{n}$ is decreasing and $\sum_{k} a_{k}^{n}<\infty$, by Remark 3.9. Moreover $\alpha^{n} \leq \alpha^{n+1}$.

We claim that there is a decreasing positive sequence $\alpha$ such that $\sum_{k} \alpha_{k}<\infty$ and for each $n$ there exists $k_{n}$ such that $\alpha_{k} \geq \alpha_{k}^{n}$ for all $k \geq k_{n}$. Then we may use Corollary 3.5 and Remark 3.9 as in the proof of Theorem 3.6 to see that $\mathcal{E}^{(M)}$ with $M_{k}:=a_{k}^{-k}$, thus also $\mathcal{E}^{(\mathfrak{M})}$, is non-quasianalytic.

It remains to show the claim; the proof is based on ideas from [82, Proposition 4.7]. We define recursively two increasing sequences of integers $\left(p_{j}\right)_{j \in \mathbb{N}}$ and $\left(q_{j}\right)_{j \in \mathbb{N}}$. Set $p_{0}=q_{0}=0$ and let

- $p_{j}$ be the minimal integer such that $p_{j}>q_{j-1}$ and $\sum_{k>p_{j}} \alpha_{k}^{j+1} \leq 2^{-j}$,

- $q_{j}$ be the minimal integer such that $\alpha_{p_{j}}^{j}>\alpha_{q_{j}+1}^{j+1}$.

This makes sense since $\alpha_{k}^{n} \rightarrow 0$ as $k \rightarrow \infty$ for each $n$. Moreover it implies $q_{j} \geq p_{j}$. Then we define

$$
\alpha_{k}:= \begin{cases}\alpha_{k}^{j} & \text { if } q_{j-1}<k \leq p_{j}, \\ \alpha_{p_{j}}^{j} & \text { if } p_{j}<k \leq q_{j} .\end{cases}
$$

By construction $\alpha$ is decreasing. By the minimality of $q_{j}$ we have $\alpha_{p_{j}}^{j} \leq \alpha_{k}^{j+1}$ for $p_{j}<k \leq q_{j}$. Thus

$$
\sum_{k} \alpha_{k}=\sum_{j}\left(\sum_{p_{j}<k \leq q_{j}} \alpha_{p_{j}}^{j}+\sum_{q_{j}<k \leq p_{j+1}} \alpha_{k}^{j+1}\right) \leq \sum_{j}\left(\sum_{p_{j}<k \leq p_{j+1}} \alpha_{k}^{j+1}\right) \leq \sum_{j} 2^{-j} .
$$

For fixed $n$ we have $a_{k} \geq a_{k}^{n}$ for all $k \geq q_{n-1}$. The claim is proved.

Theorem 11.17. Let $\omega$ be a weight function with associated weight matrix $\mathfrak{W}=$ $\left\{W^{x}\right\}_{x>0}$. The following conditions are equivalent:

(1) $\mathcal{E}^{\{\omega\}}$ is quasianalytic.

(2) $\mathcal{E}^{(\omega)}$ is quasianalytic. 
(3) The weight function $\omega$ is quasianalytic, i.e.,

$$
\int_{1}^{\infty} \frac{\omega(t)}{t^{2}} d t=\infty
$$

(4) The weight sequence $W^{x}$ is quasianalytic for all $x>0$.

(5) The weight sequence $W^{x}$ is quasianalytic for some $x>0$.

Proof. It suffices to show the equivalence of (3), (4), and (5). The rest follows from Theorem 11.16. Now $W^{x}$ is quasianalytic if and only if the associated function $\omega_{W^{x}}(t)=\sup _{k \in \mathbb{N}} \log \frac{t^{k}}{W_{k}^{x}}$ satisfies $\int_{1}^{\infty} \frac{\omega_{W^{x}}(t)}{t^{2}} d t=\infty$; cf. [47, Lemma 4.1]. Each $\omega_{W^{x}}$ is equivalent to $\omega$; cf. [74, Lemma 5.7].

Note that a non-quasianalytic weight function $\omega$ necessarily satisfies $\omega(t)=o(t)$ as $t \rightarrow \infty$, i.e., $C^{\omega}(U) \subseteq \mathcal{E}^{(\omega)}(U)$. Indeed, since $\omega$ is increasing,

$$
\frac{\omega(t)}{t}=\int_{t}^{\infty} \frac{\omega(t)}{s^{2}} d s \leq \int_{t}^{\infty} \frac{\omega(s)}{s^{2}} d s \rightarrow 0 \quad \text { as } t \rightarrow \infty .
$$

11.10. Stability properties. We state without proof a characterization of stability under composition for Braun-Meise-Taylor classes; for the general classes $\mathcal{E}^{[\mathfrak{M}]}$ we refer to [74].

Theorem 11.18 ([36], [74]). Let $\omega$ be a weight function satisfying $\omega(t)=O(t)$ as $t \rightarrow \infty$. The following conditions are equivalent.

(1) $\exists C>0 \exists t_{0}>0 \forall \lambda \geq 1 \forall t \geq t_{0}: \omega(\lambda t) \leq C \lambda \omega(t)$.

(2) $\omega$ is equivalent to a concave weight function.

(3) $\omega$ is equivalent to a subadditive weight function.

(4) $\mathcal{E}^{\{\omega\}}$ is stable under composition.

(5) $\mathcal{E}^{(\omega)}$ is stable under composition.

The condition $\omega(t)=O(t)$ as $t \rightarrow \infty$ is only used in the directions (4) $\Rightarrow$ (1) and $(5) \Rightarrow(1)$.

That subadditivity is a sufficient condition for stability under composition is seen as follows: Let $\mathfrak{W}=\left\{W^{x}\right\}_{x>0}$ be the associated weight matrix. We have

$$
W_{k}^{x}=\exp \left(\frac{1}{x} \varphi^{*}(x k)\right)=\exp \sup _{t \geq 1}\left(k \log (t)-\frac{1}{x} \omega(t)\right)=\sup _{t \geq 1}\left(t^{k} e^{-\frac{1}{x} \omega(t)}\right) .
$$

Thus subadditivity of $\omega$ implies

$$
w_{j}^{x} w_{k}^{x}=\sup _{t, s \geq 1}\left(\frac{t^{j} s^{k}}{j ! k !} e^{-\frac{1}{x}(\omega(t)+\omega(s))}\right) \leq \sup _{t, s \geq 1}\left(\frac{(t+s)^{j+k}}{(j+k) !} e^{-\frac{1}{x} \omega(t+s)}\right)=w_{j+k}^{x} .
$$

Together with Lemma 11.3(3) we see that, for all $\alpha_{i} \in \mathbb{N}_{>0}$ with $\alpha_{1}+\cdots+\alpha_{j}=k$,

$$
w_{j}^{x} w_{\alpha_{1}}^{x} \cdots w_{\alpha_{j}}^{x} \leq w_{1}^{j} w_{j}^{2 x} w_{\alpha_{1}-1}^{2 x} \cdots w_{\alpha_{j}-1}^{2 x} \leq w_{1}^{j} w_{k}^{2 x}
$$

which implies $\left(W^{x}\right)^{\circ} \preccurlyeq W^{2 x}$ for all $x>0$. From this stability under composition follows easily (cf. Section 2.5).

Remark 11.19. Let $\omega$ be a weight function with $\omega(t)=o(t)$ as $t \rightarrow \infty$. That $\omega$ is equivalent to a concave weight function is furthermore equivalent to any of the following conditions:

- There is a weight matrix $\mathfrak{S}$ consisting of strongly log-convex weight sequences such that $\mathcal{E}^{\{\omega\}}=\mathcal{E}^{\{\mathfrak{S}\}}$. 
- There is a weight matrix $\mathfrak{S}$ consisting of strongly log-convex weight sequences such that $\mathcal{E}^{(\omega)}=\mathcal{E}^{(\mathfrak{S})}$.

- $\mathcal{E}^{\{\omega\}}$ can be described by almost analytic extensions.

- $\mathcal{E}^{(\omega)}$ can be described by almost analytic extensions.

For all this (including the meaning of almost analytic extensions) we refer to [40, Theorem 4.8] and [79, Theorem 11]. Furthermore, these conditions are equivalent to the classes $\mathcal{E}^{[\omega]}$ to be stable under inverse/implicit functions and solving ODEs (in the sense described in Section 2.5), respectively; see [75].

\section{Extension in Braun-Meise-Taylor classes}

12.1. Whitney ultrajets. Let $A \subseteq \mathbb{R}^{n}$ be a closed non-empty set. Let $\omega$ be a weight function. A Whitney jet $F=\left(F^{\alpha}\right)_{\alpha \in \mathbb{N}^{n}} \in \mathcal{E}(A)$ is called a $\omega$-Whitney ultrajet of Beurling type on $A$ if for all compact subsets $K \subseteq A$ and all integers $m \geq 1$ we have

$$
\|F\|_{K, 1 / m}^{\omega}:=\sup _{x \in K} \sup _{\alpha \in \mathbb{N}^{n}}\left|F^{\alpha}(x)\right| \exp \left(-m \varphi^{*}\left(\frac{|\alpha|}{m}\right)\right)<\infty
$$

and

$$
|F|_{K, 1 / m}^{\omega}:=\sup _{\substack{x, y \in K \\ x \neq y}} \sup _{p \in \mathbb{N}} \sup _{|\alpha| \leq p}\left|\left(R_{x}^{p} F\right)^{\alpha}(y)\right| \frac{(p+1-|\alpha|) !}{|x-y|^{p+1-|\alpha|}} \exp \left(-m \varphi^{*}\left(\frac{p+1}{m}\right)\right)<\infty .
$$

We denote by $\mathcal{E}^{(\omega)}(A)$ the locally convex space of all $\omega$-Whitney ultrajets $F$ of Beurling type on $A$ equipped with the projective limit topology with respect to the system of seminorms $\|F\|_{K, 1 / m}^{\omega}+|F|_{K, 1 / m}^{\omega}$. The space of $\omega$-Whitney ultrajets of Roumieu type on $A$ is

$$
\mathcal{E}^{\{\omega\}}(A):=\left\{F \in \mathcal{E}(A): \forall K \subseteq_{c p} A \exists m \in \mathbb{N}_{\geq 1}:\|F\|_{K, m}^{\omega}+|F|_{K, m}^{\omega}<\infty\right\}
$$

supplied with its natural locally convex topology. In view of Lemma 11.3(5), we have

$$
\begin{aligned}
& \mathcal{E}^{(\omega)}(A)=\operatorname{proj}_{K \subseteq_{c p} A} \operatorname{proj}_{m>0} \mathcal{E}^{\left(W^{1 / m}\right)}(K), \\
& \mathcal{E}^{\{\omega\}}(A)=\operatorname{proj}_{K \subseteq_{c p} A} \operatorname{ind}_{m>0} \mathcal{E}^{\left\{W^{m}\right\}}(K),
\end{aligned}
$$

where $\mathfrak{W}=\left\{W^{x}\right\}_{x>0}$ is the associated weight matrix of $\omega$.

12.2. Strong weight functions. We shall see that $\mathcal{E}^{[\omega]}$ admits extension theorems preserving the class if and only if $\omega$ is a strong weight function.

Definition 12.1. A non-quasianalytic weight function $\omega$ is called strong if

$$
\exists C>0 \forall t>0: \int_{1}^{\infty} \frac{\omega(u t)}{u^{2}} d u \leq C \omega(t)+C .
$$

Lemma 12.2 ([61, Propositions 1.3 and 1.7]). Let $\omega:[0, \infty) \rightarrow[0, \infty)$ be an increasing function with $\omega(0)=0$ and $\omega(t) \rightarrow \infty$ as $t \rightarrow \infty$. The following conditions are equivalent: ${ }^{14}$

(1) $\omega$ satisfies (12.3).

\footnotetext{
${ }^{14}$ If $\omega$ satisfies these equivalent conditions, then $\omega(t)=O\left(t^{\alpha}\right)$ as $t \rightarrow \infty$ for some $0<\alpha<1$; see [61, Corollary 1.4]. That means the class contains a Gevrey class (of Roumieu or Beurling type, respectively).
} 
(2) There exist constants $K>H>1$ such that $\omega(K t) \leq H \omega(t)$ for all sufficiently large $t$.

(3) The increasing concave function

$$
\kappa(t):=\int_{1}^{\infty} \frac{\omega(u t)}{u^{2}} d u, \quad t>0
$$

satisfies (12.3) and $\omega \leq \kappa \leq C \omega+C$ for some $C>0$.

(4) The harmonic extension

$$
P_{\omega}(x+i y):= \begin{cases}\frac{|y|}{\pi} \int_{\mathbb{R}} \frac{\omega(t)}{(t-x)^{2}+y^{2}} d t & \text { if }|y|>0, \\ \omega(x) & \text { if } y=0,\end{cases}
$$

satisfies $P_{\omega}(z)=O(\omega(z))$ as $|z| \rightarrow \infty$, where $\omega(z):=\omega(|z|)$.

Example 12.3. (1) $\omega_{s}(t)=\max \left(0,(\log t)^{s}\right)$ is a strong weight function for each $s>1$.

(2) Let $M=\left(M_{k}\right)$ be a strongly regular weight sequence. Then $\omega_{M}$ satisfies (12.3), by [47, Proposition 4.4]. By Lemma 12.2(3), we have $\omega_{M} \leq \kappa \leq C \omega_{M}+C$. Since $\kappa$ is subadditive,

$$
\omega_{M}(2 t) \leq \kappa(2 t) \leq 2 \kappa(t) \leq 2 C \omega_{M}(t)+2 C,
$$

i.e., $\omega_{M}$ satisfies 11.1(1) and hence is a weight function (the other conditions are always fulfilled by $\left.\omega_{M}\right)$. Moreover, $2 \omega_{M}(t) \leq \omega_{M}(D t)+D$, as $M$ has moderate growth, see [47, Proposition 3.6]. Then Theorem 11.6 applied to $\omega_{M}$ shows that $\mathcal{E}^{[M]}=\mathcal{E}^{\left[\omega_{M}\right]}$, since $M_{k}=\sup _{t \geq 0} \frac{t^{k}}{\exp \left(\omega_{M}(t)\right)}=e^{\varphi^{*}(k)}=W_{k}^{1}$ (cf. [47, Proposition 3.2]). Consequently, the extension results for strong weight functions comprise those for strongly regular weight sequences.

12.3. The singleton and other sets with nice geometry. It was shown in [61] that Whitney ultrajets of class $\mathcal{E}^{(\omega)}$ admit extension from sets with nice geometry, including the singleton $\{0\}$.

Theorem 12.4 ([61, Theorem 3.10]). Let $\omega$ be a non-quasianalytic weight function. The following conditions are equivalent:

(1) $\omega$ is strong.

(2) $j_{\{0\}}^{\infty}: \mathcal{E}^{(\omega)}\left(\mathbb{R}^{n}\right) \rightarrow \mathcal{E}^{(\omega)}(\{0\})$ is surjective.

(3) $j_{K}^{\infty}: \mathcal{E}^{(\omega)}\left(\mathbb{R}^{n}\right) \rightarrow \mathcal{E}^{(\omega)}(K)$ is surjective for all compact convex $K \subseteq \mathbb{R}^{n}$ with non-empty interior.

(4) $j_{\bar{\Omega}}^{\infty}: \mathcal{E}^{(\omega)}\left(\mathbb{R}^{n}\right) \rightarrow \mathcal{E}^{(\omega)}(\bar{\Omega})$ is surjective for all bounded open $\Omega \subseteq \mathbb{R}^{n}$ with real analytic boundary.

Let us sketch an argument for the equivalence of (1) and (2). The FourierLaplace transform is a linear topological isomorphism between $\mathcal{E}^{(\omega)}\left(\mathbb{R}^{n}\right)_{b}^{\prime}$ and the weighted space of entire functions

$$
\mathcal{A}_{1}\left(\mathbb{C}^{n}\right):=\left\{f \in \mathcal{H}\left(\mathbb{C}^{n}\right): \exists j \in \mathbb{N}: \sup _{z \in \mathbb{C}^{n}}|f(z)| e^{-j(|\operatorname{Im} z|+\omega(z))}<\infty\right\}
$$

equipped with its natural inductive limit topology; cf. [19]. The dual $\mathcal{E}^{(\omega)}(\{0\})_{b}^{\prime}$ can be identified with $\left(\Lambda_{n}^{(\omega)}\right)_{b}^{\prime}$, where

$$
\Lambda_{n}^{(\omega)}:=\left\{\left(c_{\alpha}\right) \in \mathbb{C}^{\mathbb{N}^{n}}: \forall m \in \mathbb{N}: \sum_{\alpha}\left|c_{\alpha}\right| e^{-m \sum_{j=1}^{n} \varphi^{*}\left(\frac{\alpha_{j}}{m}\right)}<\infty\right\} .
$$


Then the map $\left(\Lambda_{n}^{(\omega)}\right)_{b}^{\prime} \ni\left(c_{\alpha}\right) \mapsto\left(z \mapsto \sum_{\alpha} c_{\alpha}(-i z)^{\alpha}\right)$ is a linear topological isomorphism between $\left(\Lambda_{n}^{(\omega)}\right)_{b}^{\prime}$ and

$$
\mathcal{A}_{2}\left(\mathbb{C}^{n}\right):=\left\{f \in \mathcal{H}\left(\mathbb{C}^{n}\right): \exists j \in \mathbb{N}: \sup _{z \in \mathbb{C}^{n}}|f(z)| e^{-j \omega(z)}<\infty\right\}
$$

and we have the commutative diagram

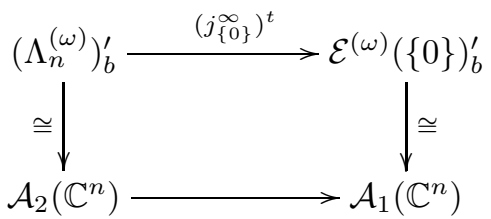

where the bottom arrow is the inclusion map. So (2) holds if and only if the inclusion $\mathcal{A}_{2}\left(\mathbb{C}^{n}\right) \rightarrow \mathcal{A}_{1}\left(\mathbb{C}^{n}\right)$ is an injective topological homomorphism. It is shown in [61] that the latter holds if and only if $P_{\omega}(z)=O(\omega(z))$ as $|z| \rightarrow \infty$; the proof relies on the Phragmén-Lindelöf principle and Hörmander's $L^{2}$-estimates for the solution of the $\bar{\partial}$-problem.

Remark 12.5. An analogous result holds in the Roumieu case $\mathcal{E}^{\{\omega\}}$, see [17].

Concerning the existence of extension operators in the above cases we have

Theorem 12.6 ([63]). Let $\omega$ be a strong weight function. Then

(1) $j_{\bar{\Omega}}^{\infty}: \mathcal{E}^{(\omega)}\left(\mathbb{R}^{n}\right) \rightarrow \mathcal{E}^{(\omega)}(\bar{\Omega})$ is split surjective for all bounded open $\Omega \subseteq \mathbb{R}^{n}$ with real analytic boundary.

(2) $j_{\{0\}}^{\infty}: \mathcal{E}^{(\omega)}\left(\mathbb{R}^{n}\right) \rightarrow \mathcal{E}^{(\omega)}(\{0\})$ is split surjective if and only if

$$
\forall C>1 \exists \delta>0 \exists t_{0}>0 \forall t \geq t_{0}: \omega^{-1}(C t) \omega^{-1}(\delta t) \leq\left(\omega^{-1}(t)\right)^{2} .
$$

A weight function $\omega$ satisfying (12.6) is called a $(D N)$-weight.

The proof of (1) is based on the splitting theorem of Vogt and Wagner [91] for the short exact sequence of nuclear Fréchet spaces

$$
0 \longrightarrow \mathcal{D}^{(\omega)}(\bar{B} \backslash \Omega) \longrightarrow \mathcal{D}^{(\omega)}(\bar{B}) \stackrel{j \frac{\infty}{\Omega}}{\longrightarrow} \mathcal{E}^{(\omega)}(\bar{\Omega}) \longrightarrow 0
$$

where $B$ is a large open ball containing $\bar{\Omega}$. By assumption, $K:=\bar{B} \backslash \Omega$ is the closure of a bounded open set with real analytic boundary and, for such $K$, the space $\mathcal{D}^{(\omega)}(K)$ has property $(\Omega)$, see [63, Corollary 2.9]. On the other hand $\mathcal{E}^{(\omega)}(\bar{\Omega})$ has property (DN), by [62, Proposition 5.7].

For the singleton, we note that $\omega$ satisfies (12.6) if and only if $\mathcal{E}^{(\omega)}(\{0\})$ has property (DN), by [60, Theorem 2.17 and Proposition 3.1]. Thus, in dimension $n=1$ the splitting theorem can be applied as above. The case $n>1$ follows by a tensor product argument; cf. $[63,3.1]$. Since $\mathcal{D}^{(\omega)}(\bar{B})$ has $(\mathrm{DN})$, by $[62$, Lemma $1.10(\mathrm{~b})]$, so does $\mathcal{E}^{(\omega)}(\{0\})$ if the sequence splits, since (DN) is inherited by closed subspaces. See also [61, Corollary 3.12].

Example 12.7. (1) If $M$ is a strongly regular weight sequence, then $\omega_{M}$ is a (DN)-weight.

(2) The weight functions $\omega_{s}(t)=\max \left\{0,(\log t)^{s}\right\}, s>1$, are strong, but not (DN)-weights. 
Remark 12.8. The function $\omega(t)=\max \{0, \log t\}$ is not a weight function, since condition 11.1(2) is violated. Nevertheless it satisfies (12.3) and violates (12.6). Then each seminorm $\|\cdot\|_{K, \rho}^{\omega}$ vanishes identically. So, formally, one may identify $\mathcal{E}^{(\omega)}(U)$ with $C^{\infty}(U)$. Indeed, the theory of extension operators in the BraunMeise-Taylor setting of Beurling type on the one hand and in the $C^{\infty}$ setting on the other hand have many similarities.

Remark 12.9. As for Denjoy-Carleman classes the Borel map is never onto in the quasianalytic case: Let $\omega$ be a quasianalytic weight function such that $\omega(t)=o(t)$ as $t \rightarrow \infty$, i.e., the real analytic class is strictly contained in $\mathcal{E}^{(\omega)}$. Then there exist elements in $\Lambda^{(\omega)}$ that are not contained in the Borel image $j_{\{0\}}^{\infty} \mathcal{E}_{0}^{\{\sigma\}}$ of any quasianalytic weight function $\sigma$. This can be deduced from Theorem 5.4 using the description of Braun-Meise-Taylor classes given in Theorem 11.4. For details see [77].

12.4. Optimal cutoff functions. As in the Denjoy-Carleman setting, to address the extension problem for general closed sets one needs cutoff functions with certain optimal estimates. Such functions are obtained by constructing certain entire functions and applying the Paley-Wiener theorem. This in turn is based on Hörmanders estimates for the solution of the $\bar{\partial}$-problem and ultimately boils down to finding subharmonic functions on $\mathbb{C}$ with suitable upper and lower bounds.

The optimal bounds are here expressed in terms of the conjugate $\omega^{\star}$ : for a function $\omega:[0, \infty) \rightarrow[0, \infty)$ with $\omega(t)=o(t)$ as $t \rightarrow \infty$ one defines

$$
\omega^{\star}(t):=\sup _{s \geq 0}(\omega(s)-s t), \quad t>0 .
$$

Then $\omega^{\star}$ is decreasing, continuous, and convex with $\omega^{\star}(t) \rightarrow \infty$ as $t \rightarrow 0$, see [69, Remark 1.5]. Since $\omega(t)=o(t)$ as $t \rightarrow \infty, \omega^{\star}(t)$ is finite for all $t$. If $\omega$ is concave and increasing, then, by [69, Proposition 1.6],

$$
\omega(t)=\inf _{s>0}\left(\omega^{\star}(s)+s t\right), \quad t>0 .
$$

Theorem 12.10 ([15, Proposition 2.2]). Let $\omega$ be a strong concave weight function. For each $n \in \mathbb{N}_{\geq 1}$ there exist $m \in \mathbb{N}_{\geq 1}, C>0$ and $0<r_{0}<\frac{1}{2}$ such that for all $0<r<r_{0}$ there exist $\varphi_{n, r} \in C^{\infty}(\mathbb{R})$ with the following properties:

(1) $0 \leq \varphi_{n, r} \leq 1$.

(2) $\left.\varphi_{n, r}\right|_{[-r, r]}=1$ and $\operatorname{supp} \varphi_{n, r} \subseteq\left[-\frac{9}{8} r, \frac{9}{8} r\right]$.

(3) We have

$$
\left\|\varphi_{n, r}\right\|_{\mathbb{R}, m}^{\omega} \leq C \exp \left(\frac{1}{n} \omega^{\star}(n r)\right) .
$$

The functions $\varphi_{n, r}$ are useful for the Roumieu case. The following cutoff functions are needed in the Beurling case.

Theorem 12.11 ([37, Corollary 2.6]). Let $\omega$ be a strong concave weight function. There exist functions $\left(\varphi_{r}\right)_{r>0}$ in $\mathcal{E}^{(\omega)}(\mathbb{R})$ such that:

(1) $0 \leq \varphi_{r} \leq 1$.

(2) $\left.\varphi_{r}\right|_{[-r, r]}=1$ and $\operatorname{supp} \varphi_{r} \subseteq\left[-\frac{9}{8} r, \frac{9}{8} r\right]$.

(3) For each $m \in \mathbb{N}_{\geq 1}$ there exist $C>0$ and $n \in \mathbb{N}_{\geq 1}$ such that for all $r>0$

$$
\left\|\varphi_{r}\right\|_{\mathbb{R}, \frac{1}{m}}^{\omega} \leq C \exp \left(n \omega^{\star}(r)\right) .
$$


In combination with Proposition 7.7 we easily obtain corresponding partitions of unity subordinate to families of Whitney cubes for a given closed set $A$.

Remark 12.12. The existence of such optimal cutoff functions is equivalent to the fact that $\omega$ is a strong weight function and additionally to the exactness of certain $\bar{\partial}$-complexes, see [53]. Recall that a strong weight function is equivalent to a strong concave weight function, by Lemma 12.2 .

\subsection{Extension of Whitney ultrajets.}

Theorem 12.13 ([15]). Let $\omega$ be a non-quasianalytic weight function. Then the following conditions are equivalent:

(1) $\omega$ is strong.

(2) $j_{A}^{\infty}: \mathcal{E}^{\{\omega\}}\left(\mathbb{R}^{n}\right) \rightarrow \mathcal{E}^{\{\omega\}}(A)$ is surjective for every closed subset $A \subseteq \mathbb{R}^{n}$.

(3) $j_{A}^{\infty}: \mathcal{E}^{(\omega)}\left(\mathbb{R}^{n}\right) \rightarrow \mathcal{E}^{(\omega)}(A)$ is surjective for every closed subset $A \subseteq \mathbb{R}^{n}$.

The sufficiency of strongness of $\omega$ for (2) is proved in analogy to Theorem 8.4: Without loss of generality one may assume that $A$ is compact. The case of the singleton yields local extensions $f_{x}, x \in A$, which form a bounded set in $\mathcal{B}^{\{\omega\}}\left(\mathbb{R}^{n}\right)$, cf. Lemma 8.2. The existence of an optimal partition of unity for a family of Whitney cubes for $A$ allows to define the required extension by a formula similar to $(8.9)$.

The Beurling case (3) can be reduced to the Roumieu case (2) by a reduction lemma (see [15]) which is similar in spirit to Lemma 8.5.

The necessity of (1) for (2) as well as for (3) follows from the special case of the singleton. But is was shown in [1] that $\omega$ must be strong, if there is any non-empty compact set $K \subseteq \mathbb{R}^{n}$ such that $j_{K}^{\infty}: \mathcal{E}^{\{\omega\}}\left(\mathbb{R}^{n}\right) \rightarrow \mathcal{E}^{\{\omega\}}(K)$ or $j_{K}^{\infty}: \mathcal{E}^{(\omega)}\left(\mathbb{R}^{n}\right) \rightarrow$ $\mathcal{E}^{(\omega)}(K)$ is surjective.

12.6. Extension operators. We saw in Theorem 12.6 that compact sets with real analytic boundary admit extension operators in the Beurling case $\mathcal{E}^{(\omega)}$ for all strong weight functions $\omega$. The singleton $\{0\}$, on the other hand, admits an extension operator if and only if $\omega$ is a strong (DN)-weight. The existence of optimal cutoff functions of Beurling type (Theorem 12.11) make it possible to extend this result to all closed sets.

Theorem 12.14 ([37, Theorem 1]). Let $\omega$ be a strong weight function. Then the following conditions are equivalent:

(1) $\omega$ is a (DN)-weight.

(2) $j_{A}^{\infty}: \mathcal{E}^{(\omega)}\left(\mathbb{R}^{n}\right) \rightarrow \mathcal{E}^{(\omega)}(A)$ is split surjective for every closed subset $A \subseteq \mathbb{R}^{n}$.

The proof is analogous to the one of Theorem 9.3. Indeed, the case of the singleton guarantees that local extension operators $E_{x}, x \in A$, exist and $\left\{E_{x}: x \in\right.$ $A\}$ is locally equicontinuous in $L\left(\mathcal{E}^{(\omega)}(A), \mathcal{E}^{(\omega)}\left(\mathbb{R}^{n}\right)\right)$. And as in Proposition 9.2 one shows that admitting an extension operator is a local property.

If $\omega$ is strong but not a (DN)-weight, then the existence of an extension operator depends on the set. In fact, if $K \subseteq \mathbb{R}^{n}$ is a non-empty compact set and $L$ a compact neighborhood of $K$, then

$$
0 \longrightarrow \mathcal{D}^{(\omega)}(L, K) \longleftrightarrow \mathcal{D}^{(\omega)}(L) \stackrel{j_{K}^{\infty}}{\longrightarrow} \mathcal{E}^{(\omega)}(K) \longrightarrow 0
$$


is an exact sequence of Fréchet spaces, by Theorem 12.13, where

$$
\mathcal{D}^{(\omega)}(L, K):=\left\{f \in \mathcal{E}^{(\omega)}\left(\mathbb{R}^{n}\right): \operatorname{supp} f \subseteq L, j_{K}^{\infty} f=0\right\}
$$

and $\mathcal{D}^{(\omega)}(L)$ carry the subspace topology of $\mathcal{E}^{(\omega)}\left(\mathbb{R}^{n}\right)$. It is shown in [37, Proposition $4.4]$ that $\mathcal{D}^{(\omega)}(L, K)$ has the property $(\Omega)$; the proof is based on the result of Meise and Taylor [63, Corollary 2.9] that $\mathcal{D}^{(\omega)}(K)$ has property $(\Omega)$ if $K$ has real analytic boundary and on the existence of optimal cutoff functions (Theorem 12.11). Thus the splitting theorem of Vogt and Wagner [91] gives

Theorem 12.15 ([37, Theorem 2]). Let $\omega$ be a strong weight function and let $K \subseteq \mathbb{R}^{n}$ be a non-empty compact set. Then the following conditions are equivalent:

(1) $\mathcal{E}^{(\omega)}(K)$ has property $(D N)$.

(2) $j_{K}^{\infty}: \mathcal{E}^{(\omega)}\left(\mathbb{R}^{n}\right) \rightarrow \mathcal{E}^{(\omega)}(K)$ is split surjective.

Compact sets that do not admit extension operators are the flat cusps

$$
K_{f}=\left\{(x, y) \in[0,1]^{2}:|y| \leq|f(x)|\right\},
$$

where $f \in \mathcal{E}^{(\omega)}(\mathbb{R})$ with $j_{\{0\}}^{\infty} f=0$. If $\omega$ is not a $(\mathrm{DN})$-weight, then $\mathcal{E}^{(\omega)}\left(K_{f}\right)$ does not have $(\mathrm{DN})$, and $j_{K}^{\infty}: \mathcal{E}^{(\omega)}\left(\mathbb{R}^{n}\right) \rightarrow \mathcal{E}^{(\omega)}(K)$ is not split surjective; see [38].

Remark 12.16. Recall that on compact sets with the Markov property, functions, that can be approximated by polynomials in an $[M]$-rapid manner, admit extensions to functions of Denjoy-Carleman type on the ambient space by a continuous linear map. But this extension involves a loss of regularity; cf. Section 9.5. In the framework of Braun-Meise-Taylor classes there is a class of weight functions, namely strong weight functions $\omega$ such that ${ }^{15}$

$$
\exists L>1 \forall t \geq 0: \omega\left(t^{2}\right) \leq L \omega(t)+L,
$$

for which the analogous problem allows a solution without loss of regularity (at least in the Beurling case), see [39]. For instance, the weight functions $\omega_{s}(t)=$ $\max \left\{0,(\log t)^{s}\right\}, s>1$, satisfy $(12.10)$.

\section{Extensions nOt PRESERVING THE ULTRADIFFERENTIABLE CLASS}

As for Denjoy-Carleman classes it is natural to ask whether the loss of regularity in the extension, for weight functions that are not strong, can be determined and controlled.

13.1. The singleton. For the singleton we have

Theorem 13.1 ([18]). Let $\omega$ be a non-quasianalytic weight function, and $\sigma$ another weight function. Then the following conditions are equivalent:

(1) $\exists C>0 \forall t>0: \int_{1}^{\infty} \frac{\omega(t u)}{u^{2}} d u \leq C \sigma(t)+C$.

(2) $\mathcal{E}^{\{\sigma\}}(\{0\}) \subseteq j_{\{0\}}^{\infty} \mathcal{E}^{\{\omega\}}\left(\mathbb{R}^{n}\right)$

(3) $\mathcal{E}^{(\sigma)}(\{0\}) \subseteq j_{\{0\}}^{\infty} \mathcal{E}^{(\omega)}\left(\mathbb{R}^{n}\right)$.

This result was extended to compact convex sets with non-empty interior, by Langenbruch [54]. The proofs are based on descriptions of the duals of the spaces at hand as weighted spaces of entire functions.

\footnotetext{
${ }^{15}$ Note that (12.10) implies that $\omega$ is strong, e.g. by Lemma 12.2 .
} 
13.2. Roumieu extension on arbitrary closed sets. The general case was recently solved in the Roumieu case:

Theorem $13.2([78,79])$. Let $\omega$ be a non-quasianalytic concave weight function and $\sigma$ a weight function with $\sigma(t)=o(t)$ as $t \rightarrow \infty$. Then the following conditions are equivalent:

(1) $\exists C>0 \forall t>0: \int_{1}^{\infty} \frac{\omega(t u)}{u^{2}} d u \leq C \sigma(t)+C$.

(2) $\mathcal{E}^{\{\sigma\}}(A) \subseteq j_{A}^{\infty} \mathcal{E}^{\{\omega\}}\left(\mathbb{R}^{n}\right)$ for each closed subset $A \subseteq \mathbb{R}^{n}$.

Let us comment on the proof (of $(1) \Rightarrow(2)$ ). For convenience we call a pair $(\sigma, \omega)$ of weight functions as in the theorem and satisfying (1) admissible. The two crucial ingredients are

- cutoff functions with bounds that reflect the condition (1),

- the extension method of Dyn'kin by Taylor approximation to higher and higher degree as $A$ is approached.

Easy modifications in the proof of Theorem 12.10 yield the desired cutoff functions:

Theorem 13.3 ([78, Proposition 4.1]). Let $(\sigma, \omega)$ be an admissible pair of weight functions. For each $n \in \mathbb{N}_{\geq 1}$ there exist $m \in \mathbb{N}_{\geq 1}, C>0$ and $0<r_{0}<\frac{1}{2}$ such that for all $0<r<r_{0}$ there exist $\varphi_{n, r} \in C^{\infty}(\mathbb{R})$ with the following properties:

(1) $0 \leq \varphi_{n, r} \leq 1$.

(2) $\left.\varphi_{n, r}\right|_{[-r, r]}=1$ and $\operatorname{supp} \varphi_{n, r} \subseteq\left[-\frac{9}{8} r, \frac{9}{8} r\right]$.

(3) We have

$$
\left\|\varphi_{n, r}\right\|_{\mathbb{R}, m}^{\omega} \leq C \exp \left(\frac{1}{n} \sigma^{\star}(n r)\right)
$$

Here $\sigma^{\star}$ is the conjugate of $\sigma$ defined in (12.7).

Note that $\kappa(t):=\int_{1}^{\infty} \frac{\omega(u t)}{u^{2}} d u, t>0$, is a (possibly quasianalytic) weight function and $(\kappa, \omega)$ is an admissible pair. If $(\sigma, \omega)$ is another admissible pair, then $\kappa(t)=$ $O(\sigma(t))$ as $t \rightarrow \infty$, i.e., $\mathcal{E}^{[\sigma]} \subseteq \mathcal{E}^{[\kappa]}$. Moreover, $\kappa$ is concave; cf. Lemma 12.2. It follows that we may assume without loss of generality that $\sigma$ is concave.

In order to implement Dyn'kin's extension procedure one uses the description of Braun-Meise-Taylor spaces of functions and jets by the associated weight matrix, see Theorem 11.4. In view of Remark 11.19 we may assume that there is a weight matrix $\mathfrak{S}=\left\{S^{x}\right\}_{x>0}$ such that $\mathcal{E}^{[\sigma]}=\mathcal{E}^{[\mathfrak{S}]}$, where each $S^{x}$ is strongly log-convex and

which entails

$$
\sup _{j, k}\left(\frac{s_{j+k}^{x}}{s_{j}^{2 x} s_{k}^{2 x}}\right)=H<\infty
$$

$$
h_{s^{x}}(t) \leq h_{s^{2 x}}(H t)^{2}, \quad t>0, x>0 .
$$

Setting $t_{k}^{x}:=\min _{0 \leq j \leq k} s_{j}^{2 x} s_{k-j}^{2 x}$ we obtain another weight matrix $\mathfrak{T}=\left\{T^{x}\right\}_{x>0}$ such that $\mathcal{E}^{[\sigma]}=\mathcal{E}^{[\mathfrak{T}]}$, where each $T^{x}$ is strongly log-convex and

$$
\left(\frac{t_{k}^{x}}{t_{k-1}^{x}}\right)_{k \geq 1}=\left(\frac{s_{1}^{2 x}}{s_{0}^{2 x}}, \frac{s_{1}^{2 x}}{s_{0}^{2 x}}, \frac{s_{2}^{2 x}}{s_{1}^{2 x}}, \frac{s_{2}^{2 x}}{s_{1}^{2 x}}, \frac{s_{3}^{2 x}}{s_{2}^{2 x}}, \frac{s_{3}^{2 x}}{s_{2}^{2 x}}, \ldots\right)
$$

which implies (cf. (7.4))

$$
2 \Gamma_{s^{2 x}}=\Gamma_{t^{x}} .
$$

Now, if $F=\left(F^{\alpha}\right)_{\alpha}$ is a Whitney ultrajet of class $\mathcal{E}^{\{\sigma\}}$ on some compact set $K \subseteq \mathbb{R}^{n}$, then there exists $x>0$ such that $F \in \mathcal{E}^{\left\{T^{x}\right\}}(K)$. Let $\left(Q_{j}\right)_{j \geq 1}$ be a family 
of Whitney cubes for $K$ and $\left(\varphi_{j, \epsilon}\right)_{j \geq 1}$ a corresponding partition of unity based on the cutoff functions from Theorem 13.3. Let $x_{j}$ be the center of $Q_{j}$ and $\hat{x}_{j} \in K$ with $\left|\hat{x}_{j}-x_{j}\right|=d_{K}\left(x_{j}\right)$. We define

$$
f(z):= \begin{cases}\sum_{j \geq 1} \varphi_{j, \epsilon}(z) T_{\hat{x}_{j}}^{p\left(x_{j}\right)} F(z) & \text { if } z \in \mathbb{R}^{n} \backslash K, \\ F^{0}(z) & \text { if } z \in K,\end{cases}
$$

where

$$
p(z):=2 \Gamma_{s^{2 x}}\left(L d_{K}(z)\right)-1
$$

and $L$ is a positive constant. Then is not difficult to mimic the steps sketched in Section 10.3 (in particular, choosing $\epsilon$ and $L$ suitably) to see that $f$ indeed provides an extension of class $\mathcal{E}^{\{\omega\}}$. The properties (13.2) and (13.3) serve as substitutes of (7.9) and (7.10). The connection between the bound (13.1) and the functions $h_{s^{x}}$ is established by the observation (see [78, Corollary 3.11])

$$
\forall x>0 \exists 0<c \leq 1 \forall t>0: e^{c \sigma^{\star}(t)} \leq \frac{e}{h_{s^{x}}(c t)} .
$$

For a detailed presentation we refer to [79].

Remark 13.4. The proof shows that, if $\mathfrak{S}$ and $\mathfrak{W}$ are weight matrices with $\mathcal{E}^{\{\mathfrak{S}\}}=$ $\mathcal{E}^{\{\sigma\}}$ and $\mathcal{E}^{\{\mathfrak{W}\}}=\mathcal{E}^{\{\omega\}}$, respectively, then for each $S \in \mathfrak{S}$ and $a>0$ there exist $W \in \mathfrak{W}, b>0$, and an extension operator $\mathcal{E}_{a}^{S}(K) \rightarrow \mathcal{E}_{b}^{W}\left(\mathbb{R}^{n}\right)$.

13.3. Beurling extension on arbitrary closed sets. We intend to reduce the Beurling to the Roumieu case (in a way similar to Theorem 10.8). So, for an admissible pair of weight functions $(\sigma, \omega)$ and for a Whitney ultrajet $F$ of class $\mathcal{E}^{(\sigma)}$, we would like to find an admissible pair of weight functions $(\tilde{\sigma}, \tilde{\omega})$ such that $F$ is also of Roumieu class $\mathcal{E}^{\{\tilde{\sigma}\}}$ and $\omega(t)=o(\tilde{\omega}(t))$ as $t \rightarrow \infty$. Then we could infer from Theorem 13.2 that $F$ has an extension of class $\mathcal{E}^{\{\tilde{\omega}\}}$ and hence of class $\mathcal{E}^{(\omega)}$, thanks to $\omega(t)=o(\tilde{\omega}(t))$ as $t \rightarrow \infty$.

Unfortunately, it is not clear how to transfer the condition

$$
\exists C>0 \forall t>0: \int_{1}^{\infty} \frac{\omega(t u)}{u^{2}} d u \leq C \sigma(t)+C
$$

to the pair $(\tilde{\sigma}, \tilde{\omega})$ (in such a way that also all the other requirements are fulfilled). But a stronger condition can be transferred: let $\omega$ be a non-quasianalytic concave weight function and $\sigma$ a weight function with $\sigma(t)=o(t)$ as $t \rightarrow \infty$. We say that the pair $(\sigma, \omega)$ is strongly admissible if

$$
\exists r \in(0,1) \exists C>0 \forall t>0: \int_{1}^{\infty} \frac{\omega(t u)}{u^{1+r}} d u \leq C \sigma(t)+C .
$$

It is obvious that a strongly admissible pair is admissible. On the other hand $\left(\omega_{\alpha-1}, \omega_{\alpha}\right)$, where $\omega_{\alpha}(t)=t(\log t)^{-\alpha}$ and $\alpha>1$, is admissible, but not strongly admissible; see [73, Example 11].

Lemma 13.5 ([73, Lemma 13]). Let $(\sigma, \omega)$ be a strongly admissible pair of weight functions and $f:[0, \infty) \rightarrow[0, \infty)$ any function satisfying $\sigma(t)=o(f(t))$ as $t \rightarrow \infty$. Then there exists a strongly admissible pair of weight functions $(\tilde{\sigma}, \tilde{\omega})$ such that

$$
\omega(t)=o(\tilde{\omega}(t)), \sigma(t)=o(\tilde{\sigma}(t)) \text {, and } \tilde{\sigma}(t)=o(f(t)) \text { as } t \rightarrow \infty .
$$


For the proof of the lemma it is crucial that (13.4) is equivalent to

$$
\exists C>0 \exists K>H>1 \exists t_{0} \geq 0 \forall t \geq t_{0} \forall j \in \mathbb{N}_{\geq 1}: \omega\left(K^{j} t\right) \leq C H^{j} \sigma(t) ;
$$

see [73, Proposition 7]. As a consequence (invoking also Lemma 12.2), the pair $(\omega, \omega)$ is strongly admissible if and only if $\omega$ is strong; see [73, Lemma 8].

Thanks to Lemma 13.5, the reduction scheme alluded to above then gives

Theorem 13.6 ([73, Theorem 2]). Let $(\sigma, \omega)$ be a strongly admissible pair of weight functions. Then for every closed $A \subseteq \mathbb{R}^{n}$ we have $\mathcal{E}^{(\sigma)}(A) \subseteq j_{A}^{\infty}\left(\mathcal{E}^{(\omega)}\left(\mathbb{R}^{n}\right)\right)$.

It is an open question if the conclusion holds for admissible pairs $(\sigma, \omega)$.

13.4. Extension operators. As a by-product we obtain an extension operator on certain subspaces of $\mathcal{E}^{(\sigma)}(A)$ with values in $\mathcal{E}^{(\omega)}\left(\mathbb{R}^{n}\right)$.

Theorem 13.7 ([73, Theorem 5]). Let $(\sigma, \omega)$ be a strongly admissible pair of weight functions. If $\tau$ is a weight function with $\sigma(t)=o(\tau(t))$ as $t \rightarrow \infty$, then for each nonempty closed subset $A \subseteq \mathbb{R}^{n}$ there is an extension operator $\mathcal{E}^{\{\tau\}}(A) \rightarrow \mathcal{E}^{(\omega)}\left(\mathbb{R}^{n}\right)$.

Applying Lemma 13.5 to $f=\tau$, yields a strongly admissible pair $(\tilde{\sigma}, \tilde{\omega})$ satisfying $\omega(t)=o(\tilde{\omega}(t)), \sigma(t)=o(\tilde{\sigma}(t))$, and $\tilde{\sigma}(t)=o(\tau(t))$ as $t \rightarrow \infty$. Let $K$ be a compact subset of $\mathbb{R}^{n}$. Then we have continuous inclusions $\mathcal{E}^{\{\tau\}}(K) \hookrightarrow \mathcal{E}^{(\tilde{\sigma})}(K)$ and $\mathcal{E}^{\{\tilde{\omega}\}}\left(\mathbb{R}^{n}\right) \hookrightarrow \mathcal{E}^{(\omega)}\left(\mathbb{R}^{n}\right)$. Composing these maps with

$$
\mathcal{E}^{(\tilde{\sigma})}(K) \hookrightarrow \mathcal{E}_{1}^{\tilde{S}}(K) \longrightarrow \mathcal{E}_{b}^{\tilde{W}}\left(\mathbb{R}^{n}\right) \hookrightarrow \mathcal{E}^{\{\tilde{\omega}\}}\left(\mathbb{R}^{n}\right)
$$

where the middle arrow is the extension operator from Remark 13.4, gives an extension operator $\mathcal{E}^{\{\tau\}}(K) \rightarrow \mathcal{E}^{(\omega)}\left(\mathbb{R}^{n}\right)$. Here $\tilde{S} \in \tilde{\mathfrak{S}}, \tilde{W} \in \tilde{\mathfrak{W}}$, and $\tilde{\mathfrak{S}}$, $\tilde{\mathfrak{W}}$ are weight matrices associated with $\tilde{\sigma}, \tilde{\omega}$, respectively. If $A$ is a closed subset of $\mathbb{R}^{n}$, then we may use a suitable partition of unity in order to construct the required extension operator.

Remark 13.8. All extensions in Sections 12 and 13 can be chosen to be analytic in the complement of $A$. This follows from a result of Schmets and Validivia [83] or by adapting the proof of Langenbruch [56, Theorem 13].

\section{REFERENCES}

[1] A. V. Abanin, On Whitney's extension theorem for spaces of ultradifferentiable functions, Math. Ann. 320 (2001), no. 1, 115-126.

[2] F. Acquistapace, F. Broglia, M. Bronshtein, A. Nicoara, and N. Zobin, Failure of the Weierstrass Preparation Theorem in quasi-analytic Denjoy-Carleman rings, Advances in Mathematics 258 (2014), 397-413.

[3] T. Bang, Om quasi-analytiske Funktioner, Ph.D. thesis, University of Copenhagen, 1946.

[4] - The theory of metric spaces applied to infinitely differentiable functions, Math. Scand. 1 (1953), 137-152.

[5] M. Baran and W. Plesniak, Markov's Exponent of Compact Sets in $\mathbb{C}^{n}$, Proc. Amer. Math. Soc. 123 (1995), no. 9, 2785-2791.

[6] A. Belotto da Silva, I. Biborski, and E. Bierstone, Solutions of quasianalytic equations, Selecta Math. (N.S.) 23 (2017), no. 4, 2523-2552.

[7] A. Beurling, Quasi-analyticity and general distributions, Lecture notes, AMS Summer Institute, Stanford, 1961.

[8] E. Bierstone and P. D. Milman, Canonical desingularization in characteristic zero by blowing up the maximum strata of a local invariant, Invent. Math. 128 (1997), no. 2, 207-302.

[9] _ Resolution of singularities in Denjoy-Carleman classes, Selecta Math. (N.S.) 10 (2004), no. 1, 1-28. 
[10] G. Björck, Linear partial differential operators and generalized distributions, Ark. Mat. 6 (1966), 351-407.

[11] J. Bochnak, Analytic functions in Banach spaces, Studia Math. 35 (1970), 273-292.

[12] J. Bochnak, J. Kollár, and W. Kucharz, Checking real analyticity on surfaces, Journal de Mathématiques Pures et Appliquées 133 (2020), 167-171.

[13] J. Boman, On the intersection of classes of infinitely differentiable functions, Ark. Mat. $\mathbf{5}$ (1963/1965), 301-309.

[14] Microlocal quasianalyticity for distributions and ultradistributions, Publ. Res. Inst. Math. Sci. 31 (1995), no. 6, 1079-1095.

[15] J. Bonet, R. W. Braun, R. Meise, and B. A. Taylor, Whitney's extension theorem for nonquasianalytic classes of ultradifferentiable functions, Studia Math. 99 (1991), no. 2, 155-184.

[16] J. Bonet, R. Meise, and S. N. Melikhov, A comparison of two different ways to define classes of ultradifferentiable functions, Bull. Belg. Math. Soc. Simon Stevin 14 (2007), 424-444.

[17] J. Bonet, R. Meise, and B. A. Taylor, Whitney's extension theorem for ultradifferentiable functions of Roumieu type, Proc. Roy. Irish Acad. Sect. A 89 (1989), no. 1, 53-66.

[18] - On the range of the Borel map for classes of nonquasianalytic functions, Progress in functional analysis (Peñiscola, 1990), North-Holland Math. Stud., vol. 170, North-Holland, Amsterdam, 1992, pp. 97-111.

[19] R. W. Braun, R. Meise, and B. A. Taylor, Ultradifferentiable functions and Fourier analysis, Results Math. 17 (1990), no. 3-4, 206-237.

[20] J. Bruna, An extension theorem of Whitney type for non-quasi-analytic classes of functions, J. London Math. Soc. (2) 22 (1980), no. 3, 495-505.

[21] _ On inverse-closed algebras of infinitely differentiable functions, Studia Math. 69 (1980/81), no. 1, 59-68.

[22] T. Carleman, Sur le calcul effectif d'une fonction quasi analytique dont on donne les dérivées en un point., C. R. Acad. Sci. Paris 176 (1923), 64-65 (French).

[23] _ Sur les fonctions indéfiniment dérivables., C. R. Acad. Sci., Paris 177 (1923), 422424 (French).

[24] _ Sur les fonctions quasi-analytiques., 5. Kongreß der Skandinav. Mathematiker in Helsingfors, 4.-7. Juli 1922. Helsingfors: Akadem. Buchh., 181-196 (1923)., 1923.

[25] L. Carleson, On universal moment problems., Math. Scand. 9 (1961), 197-206.

[26] H. Cartan, Sur les classes de fonctions définies par des inégalités portant sur leurs dérivées successives, Actual. Sci. Ind., no. 867, Hermann et Cie., Paris, 1940.

[27] J. Chaumat and A.-M. Chollet, Surjectivité de l'application restriction à un compact dans des classes de fonctions ultradifférentiables, Math. Ann. 298 (1994), no. 1, 7-40.

[28] _ Propriétés de l'intersection des classes de Gevrey et de certaines autres classes, Bull. Sci. Math. 122 (1998), no. 6, 455-485.

[29] Division par un polynôme hyperbolique, Canad. J. Math. 56 (2004), no. 6, 1121-1144.

[30] C. L. Childress, Weierstrass division in quasianalytic local rings, Canad. J. Math. 28 (1976), no. $5,938-953$.

[31] P. J. Cohen, A simple proof of the Denjoy-Carleman theorem, Amer. Math. Monthly $\mathbf{7 5}$ (1968), 26-31.

[32] A. Denjoy, Sur les fonctions quasi-analytiques de variable réelle, C. R. Acad. Sci. Paris 173 (1921), 1320-1322.

[33] E. M. Dyn'kin, Pseudoanalytic extension of smooth functions. The uniform scale., Transl., Ser. 2, Am. Math. Soc. 115 (1980), 33-58 (English).

[34] The pseudoanalytic extension, J. Anal. Math. 60 (1993), 45-70.

[35] A. Elkhadiri and H. Sfouli, Weierstrass division theorem in quasianalytic local rings, Studia Math. 185 (2008), no. 1, 83-86.

[36] C. Fernández and A. Galbis, Superposition in classes of ultradifferentiable functions, Publ. Res. Inst. Math. Sci. 42 (2006), no. 2, 399-419.

[37] U. Franken, Continuous linear extension of ultradifferentiable functions of Beurling type, Mathematische Nachrichten 164 (1993), no. 1, 119-139.

[38] _ Examples of compact sets with non-empty interior which do not admit a continuous linear extension operator for ultradifferentiable functions of Beurling type, Archiv der Mathematik 62 (1994), no. 3, 239-247.

[39] _ Extension of functions with $\omega$-rapid polynomial approximation, Journal of Approximation Theory 82 (1995), no. 1, 88-98. 
[40] S. Fürdös, D. N. Nenning, A. Rainer, and G. Schindl, Almost analytic extensions of ultradifferentiable functions with applications to microlocal analysis, J. Math. Anal. Appl. 481 (2020), no. 1, doi:10.1016/j.jmaa.2019.123451.

[41] A. Gorny, Contribution à l'étude des fonctions dérivables d'une variable réelle, Acta Math. 71 (1939), 317-358.

[42] J. Hadamard, Sur la généralisation de la notion de fonction analytique, Bull. Soc. Math. France 40 (1912), 28-29, (supplément spécial: vie de la société, séance du 28 février 1912).

[43] M. R. Hestenes, Extension of the range of a differentiable function, Duke Mathematical Journal 8 (1941), no. 1, 183-192.

[44] L. Hörmander, The analysis of linear partial differential operators. I, Grundlehren der Mathematischen Wissenschaften [Fundamental Principles of Mathematical Sciences], vol. 256, Springer-Verlag, Berlin, 1983, Distribution theory and Fourier analysis.

[45] E. Y. Jaffe, Pathological phenomena in Denjoy-Carleman classes, Canad. J. Math. 68 (2016), no. $1,88-108$

[46] A. Kiro, Taylor coefficients of smooth functions, Journal d'Analyse Mathématique 142 (2020), no. 1, 193-269.

[47] H. Komatsu, Ultradistributions. I. Structure theorems and a characterization, J. Fac. Sci. Univ. Tokyo Sect. IA Math. 20 (1973), 25-105.

[48] A. Kriegl, P. W. Michor, and A. Rainer, The convenient setting for non-quasianalytic Denjoy-Carleman differentiable mappings, J. Funct. Anal. 256 (2009), 3510-3544.

[49] — The convenient setting for quasianalytic Denjoy-Carleman differentiable mappings, J. Funct. Anal. 261 (2011), 1799-1834.

[50] _ The convenient setting for Denjoy-Carleman differentiable mappings of Beurling and Roumieu type, Rev. Mat. Complut. 28 (2015), no. 3, 549-597.

[51] M. Langenbruch, Extension of ultradifferentiable functions of Roumieu type, Arch. Math. (Basel) 51 (1988), no. 4, 353-362.

[52] _ Ultradifferentiable functions on compact intervals., Math. Nachr. 140 (1989), 109126 (English).

[53] Differentiable functions and the $\bar{\partial}$-complex, Functional analysis. Proceedings of the Essen Conference, held in Essen, Germany, November 24 - 30, 1991, New York, NY: Dekker, 1994, pp. 415-434 (English).

[54] _ Extension of ultradifferentiable functions, Manuscripta Math. 83 (1994), no. 2, 123143.

[55] - Analytic extension of smooth functions, Results in Mathematics 36 (1999), no. 3-4, $281-296$.

[56] - A general approximation theorem of Whitney type, RACSAM Rev. R. Acad. Cienc. Exactas Fís. Nat. Ser. A Mat. 97 (2003), no. 2, 287-303.

[57] S. Mandelbrojt, Sur les fonctions indéfiniment dérivables, Acta Math. 72 (1940), 15-29.

[58] - Séries adhérentes, régularisation des suites, applications, Gauthier-Villars, Paris, 1952.

[59] W. Matsumoto, Characterization of the separativity of ultradifferentiable classes, J. Math. Kyoto Univ. 24 (1984), no. 4, 667-678.

[60] R. Meise and B. A. Taylor, Splitting of closed ideals in (DFN)-algebras of entire functions and the property (DN), Trans. Am. Math. Soc. 302 (1987), 341-370 (English).

[61] - Whitney's extension theorem for ultradifferentiable functions of Beurling type, Ark. Mat. 26 (1988), no. 2, 265-287.

[62] _ A decomposition lemma for entire functions and its applications to spaces of ultradifferentiable functions, Mathematische Nachrichten 142 (1989), no. 1, 45-72.

[63] Linear extension operators for ultradifferentiable functions of Beurling type on compact sets, Amer. J. Math. 111 (1989), no. 2, 309-337.

[64] R. Meise and D. Vogt, Introduction to functional analysis, Oxford Graduate Texts in Mathematics, vol. 2, The Clarendon Press Oxford University Press, New York, 1997.

[65] F. Nazarov, M. Sodin, and A. Volberg, Lower bounds for quasianalytic functions. I. How to control smooth functions, Math. Scand. 95 (2004), no. 1, 59-79.

[66] D. N. Nenning, A. Rainer, and G. Schindl, Nonlinear conditions for ultradifferentiability, (2021).

[67] A. Parusiński and J.-P. Rolin, A note on the Weierstrass preparation theorem in quasianalytic local rings, Canadian Mathematical Bulletin 57 (2014), no. 3, 614-620. 
[68] H.-J. Petzsche, On E. Borel's theorem, Math. Ann. 282 (1988), no. 2, 299-313.

[69] H.-J. Petzsche and D. Vogt, Almost analytic extension of ultradifferentiable functions and the boundary values of holomorphic functions, Math. Ann. 267 (1984), no. 1, 17-35.

[70] W. Pleśniak, Markov's inequality and the existence of an extension operator for $C^{\infty}$ functions, Journal of Approximation Theory 61 (1990), no. 1, 106-117.

[71] Extension and polynomial approximation of ultradifferentiable functions in $\mathbb{R}^{N}$, Bull. Soc. R. Sci. Liège 63 (1994), no. 5, 393-402 (English).

[72] A. Rainer, Quasianalytic ultradifferentiability cannot be tested in lower dimensions, Bull. Belg. Math. Soc. Simon Stevin 26 (2019), 505-517.

[73] On the extension of Whitney ultrajets of Beurling type, Results Math. (2021), Article Number 36, https://doi.org/10.1007/s00025-021-01347-z.

[74] A. Rainer and G. Schindl, Composition in ultradifferentiable classes, Studia Math. 224 (2014), no. 2, 97-131.

[75] — Equivalence of stability properties for ultradifferentiable function classes, Rev. R. Acad. Cienc. Exactas Fis. Nat. Ser. A Math. RACSAM. 110 (2016), no. 1, 17-32.

[76] _ Extension of Whitney jets of controlled growth, Math. Nachr. 290 (2017), no. 14-15, 2356-2374, doi:10.1002/mana.201600321.

[77] _ On the Borel mapping in the quasianalytic setting, Math. Scand. 121 (2017), 293310.

[78] _ On the extension of Whitney ultrajets, Studia Math. 245 (2019), no. 3, 255-287.

[79] — On the extension of Whitney ultrajets, II, Studia Math. 250 (2020), no. 3, 283-295.

[80] J.-P. Rolin, P. Speissegger, and A. J. Wilkie, Quasianalytic Denjoy-Carleman classes and o-minimality, J. Amer. Math. Soc. 16 (2003), no. 4, 751-777 (electronic).

[81] W. Rudin, Division in algebras of infinitely differentiable functions, J. Math. Mech. 11 (1962), 797-809.

[82] G. Schindl, Characterization of ultradifferentiable test functions defined by weight matrices in terms of their Fourier transform, Note di Matematica 36 (2016), no. 2, 1-35, doi:10.1285/i15900932v36n2p1.

[83] J. Schmets and M. Valdivia, Analytic extension of ultradifferentiable Whitney jets, Collect. Math. 50 (1999), no. 1, 73-94.

[84] - Extension maps in ultradifferentiable and ultraholomorphic function spaces, Studia Math. 143 (2000), no. 3, 221-250.

[85] _ On certain extension theorems in the mixed Borel setting, J. Math. Anal. Appl. 297 (2004), no. 2, 384-403, Special issue dedicated to John Horváth.

[86] J. Siciak, A characterization of analytic functions of $n$ real variables, Studia Math. 35 (1970), 293-297.

[87] E. M. Stein, Singular integrals and differentiability properties of functions, Princeton Mathematical Series, No. 30, Princeton University Press, Princeton, N.J., 1970.

[88] V. Thilliez, On quasianalytic local rings, Expo. Math. 26 (2008), no. 1, 1-23.

[89] _ Smooth solutions of quasianalytic or ultraholomorphic equations, Monatsh. Math. 160 (2010), no. 4, 443-453.

[90] D. Vogt, Eine Charakterisierung der Potenzreihenräume von endlichem Typ und ihre Folgerungen, Manuscripta Math. 37 (1982), no. 3, 269-301.

[91] D. Vogt and M. Wagner, Charakterisierung der Quotientenräume von s und eine Vermutung von Martineau, Studia Mathematica 67 (1980), no. 3, 225-240.

[92] H. Whitney, Analytic extensions of differentiable functions defined in closed sets, Trans. Amer. Math. Soc. 36 (1934), no. 1, 63-89.

[93] D. V. Widder, The Laplace Transform, Princeton Mathematical Series, v. 6, Princeton University Press, Princeton, N. J., 1941.

Fakultät für Mathematik, Universität Wien, Oskar-Morgenstern-Platz 1, A-1090 Wien, Austria

Email address: armin.rainer@univie.ac.at 